\title{
WestVirginiaUniversity
}

THE RESEARCH REPOSITORY @ WVU

Graduate Theses, Dissertations, and Problem Reports

2009

\section{Historical response rates, reinforcement context, and behavioral persistence}

Chata A. Dickson

West Virginia University

Follow this and additional works at: https://researchrepository.wvu.edu/etd

\section{Recommended Citation}

Dickson, Chata A., "Historical response rates, reinforcement context, and behavioral persistence" (2009). Graduate Theses, Dissertations, and Problem Reports. 2881.

https://researchrepository.wvu.edu/etd/2881

This Dissertation is protected by copyright and/or related rights. It has been brought to you by the The Research Repository @ WVU with permission from the rights-holder(s). You are free to use this Dissertation in any way that is permitted by the copyright and related rights legislation that applies to your use. For other uses you must obtain permission from the rights-holder(s) directly, unless additional rights are indicated by a Creative Commons license in the record and/ or on the work itself. This Dissertation has been accepted for inclusion in WVU Graduate Theses, Dissertations, and Problem Reports collection by an authorized administrator of The Research Repository @ WVU.

For more information, please contact researchrepository@mail.wvu.edu. 
Historical Response Rates, Reinforcement Context, and Behavioral Persistence

Chata A. Dickson

Dissertation submitted to the

Eberly College of Arts and Sciences

at West Virginia University

in partial fulfillment of the requirements

for the degree of

Doctor of Philosophy

in

Psychology

K. Andy Lattal, Ph.D., Chair

Michael Perone, Ph.D.

Karen Anderson, Ph.D.

Tracy Morris, Ph.D.

Daniel Hursh, Ph.D.

Department of Psychology

Morgantown, West Virginia

2009

Keywords: Behavioral History; Behavioral Momentum; Reinforcement Context 


\section{ABSTRACT \\ Historical Response Rates, Reinforcement Context, and Behavioral Persistence}

\section{Chata A. Dickson}

In several studies, historically lower-rate responding has been found to be more persistent than historically higherrate responding (e.g., Lattal, 1989; Nevin, 1974). In these studies, historical response-rate differences were generated by reinforcement schedules (e.g., fixed-ratio [FR] and differential-reinforcement-of-low-rates-ofresponding $[\mathrm{DRL}])$ that directly affect response rate. The current study was conducted with pigeons pecking keys to determine whether this finding would hold when response-rate differences were generated by the context of reinforcement, such as a concurrently available source of reinforcement or schedule of response-independent food. In each of 3 experiments, a history-building condition was followed by a history-testing condition. In Experiment 1 , history building consisted of a multiple-concurrent (variable interval [VI] 40-s VI 20-s, VI 40-s VI 80-s) schedule, and history testing consisted of a multiple fixed interval (FI) 40-s, FI 40-s. It was expected that response rate would be lower on the VI 40-s schedule that was paired with the VI 20-s than the VI 40-s that was paired with the VI 80-s (cf. Belke, 1992), however, large differences in response rate on the two VI 40-s schedules were not obtained. The rate and temporal distribution of historically lower-rate responding initially changed more rapidly than did higherrate responding in each of the four pigeons. In attempt to increase the difference in response rate across the two components of the multiple schedule, in Experiment 2, history building consisted of a multiple concurrent (VI 40-s Tandem VI FR, VI 40-s Tandem VI DRL), and history testing consisted of a multiple (FI 40-s, FI 40-s). Schedule values were adjusted in attempt to obtain response-rate differences, but the programmed rate of reinforcement was always equal on the VI 40-s alternative across the two components. Again, large differences in response rate on the two VI 40-s schedules were not obtained. The rate of historically lower-rate responding changed more rapidly in three of the four pigeons, and the temporal distribution of historically lower-rate responding changed more gradually than did higher-rate responding. In Experiment 3, history building consisted of a multiple mixed VI 30-s variable time (VT) 30-s, VI 30-s schedule, and history testing consisted of a multiple FI 30-s, FI 30-s. The VT schedule provided the contextual schedule designed to reduce response rate in the same component. In this case, larger response-rate differences across the two components were obtained. Lower-rate responding was less persistent, both in terms of rate and temporal distribution of responding. Results are discussed in terms of factors shown to determine resistance to change, and the importance of understanding relations between schedules of reinforcement and resultant response organization. 


\section{Dedication}

This dissertation is dedicated to my parents, Daryl and Margo Dickson, who have loved me, encouraged me, and wanted me to be happy each day of my life. 


\section{Acknowledgements}

I would like to acknowledge everyone who contributed to me and to all that I have learned during my time at West Virginia University and along the journey that led me here. During my time in graduate school I was incredibly fortunate to work with faculty members who continue to serve as models for me along a number of continua. I strive to mentor as Andy Lattal, instruct as Mike Perone, and keep the focus on socially important issues as Phil Chase. It was an absolute privilege to be a student in the behavior analysis program at WVU, and I would not trade the experience for anything.

I appreciate the time and effort each of my committee members -- Andy Lattal, Mike Perone, Karen Anderson, Tracy Morris, and Dan Hursh -- allocated to reading and considering the work this dissertation comprises. I especially appreciate Karen Anderson's generosity in allowing me to run experimental sessions in her lab. Karen and her students, James Diller, Natalie Bruner, and Jon Slezak, were invariably welcoming and supportive as I set up shop in their space for several hours a day for the greater part of a year.

There are several other individuals whose support has been invaluable. Mrs. Swinney kept me on top of the tasks that were required for getting through the milestones of the program and to graduation. Rachel Detlor was quick to assist when I asked for help ranging from caring for my aging Golden Retriever, Marley, to distributing copies of my dissertation after I had relocated to Massachusetts. I am lucky to count her among the many wonderful people I met and befriended during my time in Morgantown.

I received financial support at WVU from the Swiger Fellowship, the Alumni Fund, the Office of Academic Affairs, the Eberly College of Arts and Sciences, and the Department of Psychology. I appreciate very much this support and did my best to take advantage of the opportunities it provided me to spend my time learning about, conducting, and sharing research. The support from these funds made it possible for me to maximize the learning and work that I did during my time at WVU.

I also thank my fellow graduate students for providing an intellectually stimulating environment - I will remember many details of the discussions we had in lab meetings and courses, and I can only hope that the issues with which we grappled will continue to be discussed by my students and peers in the coming years. I learned a great deal from the time spent with my cohort in the Lattal Lab, where I spent the better part of my time. I feel like it's never acknowledged quite enough how much senior students contribute to newbies in the lab. I appreciate all that I learned in the lab from Raquel Alo, Megan (Meginley) Maxwell, and Mirari Elcoro; these three taught me everything I needed to know from how to program an experiment and manage data using a computer than ran on Windows 98 to how to get a pigeon safely from the animal quarters to the lab using only a kool-aid pitcher and a little bit of patience. These three were great friends to me as they and were quick to provide constructive comments and encouragement as I interacted with the contingencies of the program and the dissertation in particular. I treasure the marathon study and writing sessions with Mirari, the challenging and enlightening late-night conversations with Harold Lobo, and the productive and enjoyable collaboration with Yusuke Hayashi. These three are among my dearest friends, and I hope these conversations and collaborations will continue for a long time.

There are many people to thank, as I have been extremely fortunate to have been influenced and supported by so many amazing people. There are a number of people who made it possible for me to get to this point. The first of these is Maria Ruiz. By the end of the first class period of Maria's Learning course at Rollins College, it was clear to me that I would spend the rest of my career in one way or another as a behavior analyst. Maria's influence on my thinking is immeasurable; she is truly my intellectual parent. Hanging around Maria as an undergrad led me to Eb Blakely at Threshold and Quest Kids. Eb was an amazing teacher; along with any amount of support I requested he gave me the freedom to learn about teaching by contacting the contingencies and then was there to help me interpret what had happened and to gently shape my repertoire. Over the last 15 years or so I have struggled to find a way to thank Maria and $\mathrm{Eb}$ for time and care they put into my development.

At the New England Center for Children and in the MABA program at Northeastern University my good fortune continued. Though by this time I was pretty sure I knew everything, I was pleased to find out in Bill Ahearn's Learning class there was still much I didn't know. Bill is a model instructor and a good friend. On the research end I worked with Murray Sidman and with Bill Dube at the Shriver Center. I could not dream up a better learning environment for myself. From each interaction with Murray I have come away with a new idea, profound to me, that has changed my perspective in some way. In Bill Dube I have a model of how to write, how to approach research questions and data, and perhaps most importantly, how to treat people. 
By the time I was in Massachusetts I had no specific plans to pursue a doctoral degree; until Caio Miguel's visit to the New England Center. Caio was bright and enthusiastic as he talked about his experience in the graduate program he was then completing. Talking with him, I was clear that this to be the next step for me. Months later I was delighted when I found that the program at WVU was a perfect match.

I am pleased now to be on the other side of the graduate school experience. I am grateful to Vinnie Strully, Kathy Foster, and all of my friends at the New England Center for having encouraged me to go to WVU, and for welcoming me back.

I thank my parents, to whom this accomplishment is dedicated, and to the rest of my family who understood, or at least took my word for it, when I had to choose lab pigeons over holiday turkey (though never for dinner).

Finally, I thank Magda, the person most directly affected by my decision to pursue graduate study in West Virginia. I don't know how she did it, but Magda accepted generously my plan to take a 3-year expedition to study in a place 600 miles from home; though I was far away, she was by my side the entire time. Magda's support kept me going through the points of exhaustion that every graduate student encounters at some time or another, and her love gave me a reason to get my work done and get home!

I am so fortunate to have had such a great life so far. Thanks to all of you and to the many others who have been an important part of my journey! 
Table of Contents

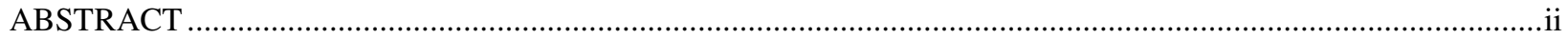

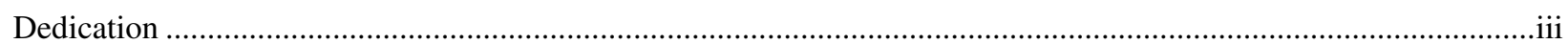

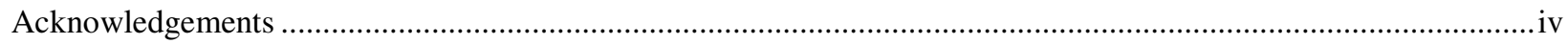

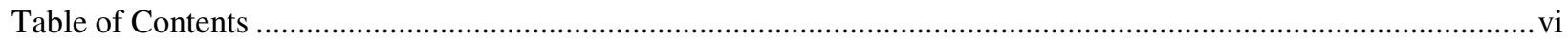

Chapter 1: Introduction and Literature Review …….....................................................................................

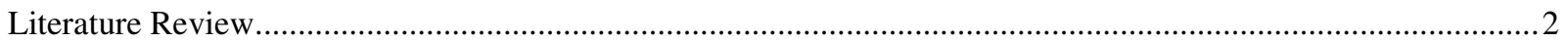

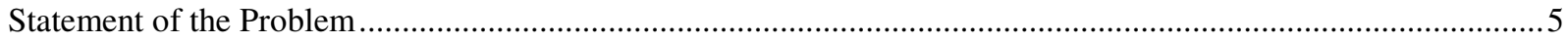

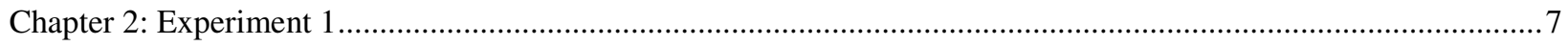

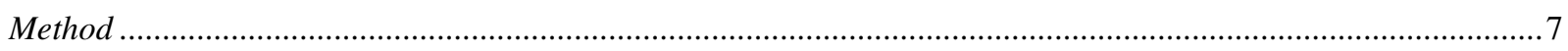

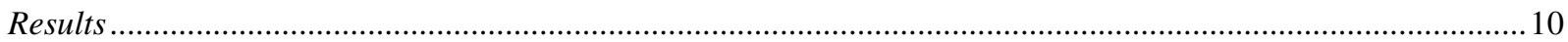

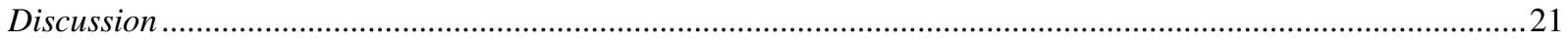

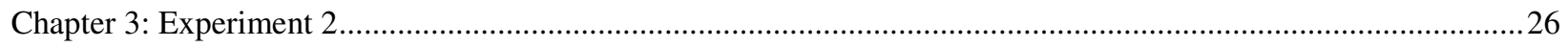

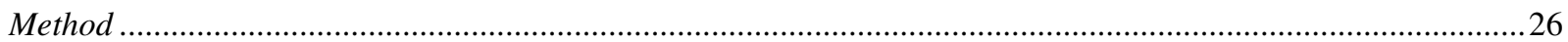

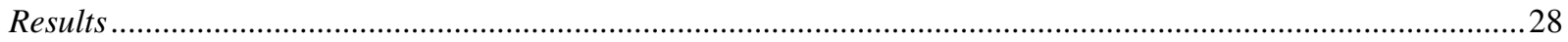

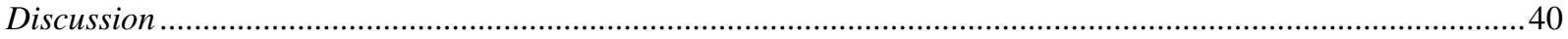

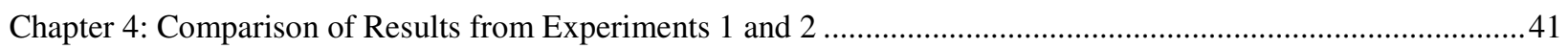

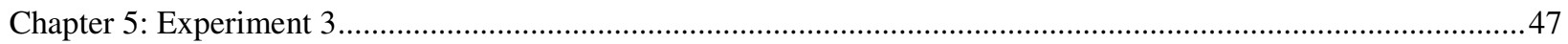

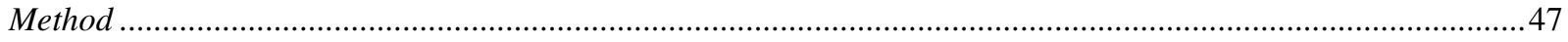

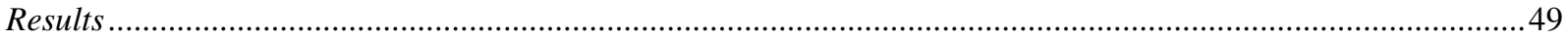

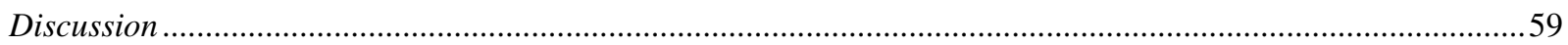

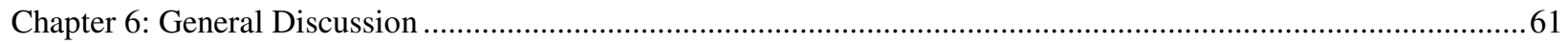

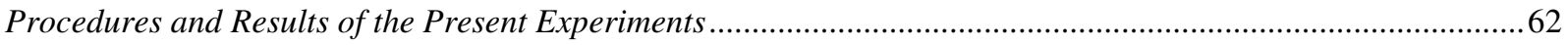

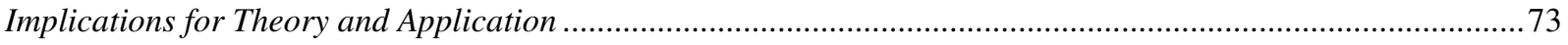

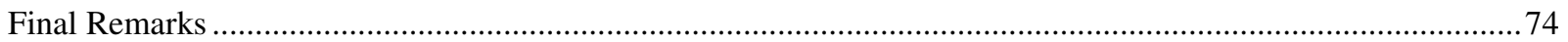

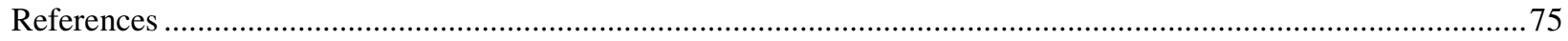

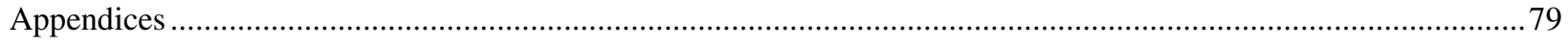


Appendix A: Component Sequences for History Building and History Testing ..............................................79

Appendix B: Adjusting Schedule Values for the History-Building Condition in Experiment 1 .............................8 80

Appendix C: Adjusting Schedule Values for the History-Building Condition in Experiment 2 ...........................84

Appendix D: Adjusting Schedule Values for the History-Building Condition in Experiment 3 ...........................8 88 
Chapter 1: Introduction and Literature Review

\section{Historical Response Rates, Reinforcement Context, and Behavioral Persistence}

A fundamental assumption of science is determinism, the premise that events occurring in space and time determine all other natural events. The behavior of organisms is among these determined events. Thus, the science of behavior predicates the assumption that environmental events, present and past, determine behavior. Most experimenters attempting to elucidate relations between behavior and other events manipulate variables that overcome the potential influence of uncontrolled factors. In behavioral studies typical of those published in peer-reviewed journals, the control between environmental manipulations and behavior is clear, and results are replicated in the behavior of additional subjects. Frequently, however, strong current environment-behavior relations are seen in some, but not all experimental subjects. When such current contingencies of reinforcement cannot account for anomalous results, prior or historical contingencies commonly are invoked as a potential source of the between-subject variation. Although it is logical to suspect that an organism's learning history may be responsible for individual differences in response to an experimental condition or treatment, it is uncommon that investigators are aware of the details of these historical contingencies. Even when researchers are aware of an organism's behavioral history, there is a paucity of research to guide their interpretations. Because behavioral history is so commonly held responsible for variations in behavior from predictions or for variability of behavior across time or organisms, it is important to investigate the ways in which history can affect behavior.

An early instance of attributing individual differences to learning history is found in an experiment conducted by Holland (1958). He found that on fixed-ratio (FR) schedules, some human subjects respond in a characteristic breakand-run pattern, while others respond more steadily throughout the interval. Holland acknowledged the factors leading to these differences were "beyond the control of the experimenter" (p. 181). In early studies that investigated the behavioral effects of prior experience subjects were exposed to different schedules of reinforcement and behavior was measured in subsequent tests (e.g., Weiner, 1964, 1969). Weiner (1969) showed that prior history of reinforcement affected the response rate of human subjects on fixed-interval (FI) schedules. In Wiener's (1969) study, when subjects responded on schedules of reinforcement that generate low rates of responding (Differential reinforcement of low rates of Responding; DRL), response rates were lower in subsequent sessions in which an FI schedule was in effect. Likewise, when subjects responded on schedules that generate high rates of responding (in this case, FR schedules) response rates were higher in subsequent FI sessions. The experience with a DRL schedule resulted in relatively lower rates on subsequent FI schedules even when several sessions of FR schedules, with their accompanying high response rates, separated these two conditions. Since Weiner's work with humans in the 1960s, history effects have been studied in more controlled settings with experimentally naïve laboratory animals (e.g., Barrett, 1977; Freeman \& Lattal, 1992; Okouchi \& Lattal, 2006; Urbain, Poling, Millam, \& Thompson, 1978). Over three decades have passed since Weiner's initial investigations of behavioral history, and still relatively little is known about factors that determine history effects. Many questions remain, for example: what are the key features of experiences that result in more or less persistent changes in behavior? In what kind of setting will learning history be the most or least evident? Studies of behavioral history address these questions.

History effects have been described as transition states (Sidman, 1960). This description emphasizes the temporary nature of these effects, and supports the rationale of experiments that manipulate variables designed to overwhelm effects of history and other uncontrolled variables. Sidman also acknowledged that when effects of prior experience are longer lived it is important to examine variables responsible for these more gradual changes. Extreme examples of persistent history effects are easy to imagine, for example, when a history of punishment decreases the likelihood of a particular response, or when history of reinforcement leads to new behavior that increases access to previously inaccessible reinforcers (e.g., reading). Experiences like these can lead to lasting or even irreversible effects on behavior. Although history effects are thought by many researchers to be temporary, and therefore of marginal importance, what is interesting about history effects is not their evanescence, but their persistence (Freeman \& Lattal, 1992). Investigating factors that affect the durability of history effects may lead to advances not only in the interpretation of experimental findings, but also for education and treatment in human applications. For example, the discovery of factors that lead to more durable desired behavior or less durable undesired behavior could lead to innovations in education and treatment of persons with problem behavior. With a better understanding of events that can lead to difference in behavioral persistence will come a better understanding of variability that occurs in behavior across organisms and across time.

An enduring consideration in the study of behavioral history is the relative contributions of the historical reinforcement rate and response rate to subsequent behavior. The relative influence of reinforcement rate and response rate are difficult to separate, but in several studies attempts have been made to control for one or the other 
of these variables. One of these studies, Freeman and Lattal (1992), was innovative in its use of multiple schedules of reinforcement to build two different behavioral histories within the same experimental condition and within the same subject. Freeman and Lattal held the overall rate of reinforcement constant across the two components of a multiple schedule, but used schedules that engendered different response rates (e.g., tandem variable-interval [VI] FR schedule in one component, and a tandem VI DRL in the other). Behavioral history in this experiment was controlled by the stimuli correlated with each component of the multiple schedule. Although in subsequent testing the schedules of reinforcement in each component were equated, differential responding persisted, in some cases for up to 60 sessions, as it had in the history-building condition. Freeman and Lattal were the first to examine behavioral history under stimulus control, and thus to show that contextual factors can affect responding in differentially persistent ways under new schedules of reinforcement.

The present study sought to explicate some of the variables of behavioral history that affect subsequent responding. Multiple schedules of reinforcement were used in attempt to generate different rates of responding under the control of schedule-correlated stimuli. The rate of reinforcement was equal across components; and effects on subsequent responding were examined. Extending Freeman and Lattal (1992), in which a history of high- and low-rate responding was constructed by differential reinforcement in the two components, the present study employed schedules of reinforcement designed to build response-rate differences in two identical schedules by manipulating contextual variables. To that end, the following literature review surveys research on behavioral history and research relevant to a series of context manipulations, and describes procedures designed to manipulate response rates in context without manipulating rate-controlling contingencies such as DRH or DRL.

\section{Literature Review}

\section{Behavioral History, Response Rate, and Reinforcement Rate}

A relatively small number of experiments has sought to specify effects on current behavior of historical behaviorenvironment relations. In these studies of behavioral history, there is a comparison between responding on two different schedules of reinforcement (hereafter, the history-building condition), and a subsequent comparison between the persistence of this responding when these schedules are both changed to a third schedule of reinforcement (hereafter, the history-testing condition). Wiener's $(1964,1969)$ studies of behavioral history were the first designed to systematically expose subjects to contingencies that had a lasting effect on behavior under novel contingencies. Although Weiner's work demonstrated empirically that learning history affects subsequent performance, the variables that contributed to the effects of this history were convoluted. Features of both the history-building condition and the history-testing condition affect the persistence of responding in history testing. Potentially influential features of each of these conditions are discussed below.

Behavioral history researchers who have sought to parse the variables responsible for enduring history effects have controlled for the potential confound of historical response rate with historical reinforcement rate. These researchers have selected contingencies that minimize the difference between the rates of reinforcement and maximize the difference between rates of responding. In most of these cases, the purpose of this control has been to allow the researcher to examine effects of response rate on subsequent behavior. For example, in a study of the interaction of behavioral history and drug effects, Urbain et al. (1978) established high- or low-rate responding while controlling for reinforcement rate in two groups of rats. Bar pressing was reinforced on an FR 40-s schedule for one group, and on a DRL (interresponse time [IRT] $>11 \mathrm{~s}$ ) schedule for the other. After fifty history-building sessions, both groups were exposed to FI 15-s schedules. Higher response rates persisted for the group that had been exposed to the FR schedule, and lower response rates persisted for the DRL-exposed group. Response-rate differences between the groups, while gradually shifting toward convergence, persisted throughout the 59 subsequent FI 15-s sessions. The authors reported two between-group differences in responding on the FI schedule: (a) the rate of responding changed more quickly in the higher-response-rate group, and (b) a positively accelerated response pattern characteristic of FI responding developed more quickly for the lower-response-rate group. Although the primary purpose of Urbain et al. was to investigate behavioral history as a modulator of drug - behavior relations, the study was an important step in explicating variables responsible for behavioral history effects.

Prior to Freeman and Lattal (1992), studies of behavioral history employed group designs. The use of within-subject methods emphasizes analysis of the behavior of the individual organism. This focus is perhaps especially important in the study of behavioral history, because history frequently is used to account for individual differences in responding. Freeman and Lattal used multiple schedules of reinforcement to build parallel behavioral histories in individual pigeons, and within individual sessions (1992, Experiment 3). These behavioral histories were "parallel" because the histories were built separately, but within the same experimental condition. In multiple schedules two or 
more components alternate, and each is associated with a different stimulus condition. In an extension of Freeman and Lattal (1992), Metzger (1992, Experiment 1) exposed pigeons to two alternating history-building components in a multiple schedule. One component maintained high rates of responding (tandem VI FR 10), and the other maintained low rates of responding (tandem VI DRL 5-s). These two components resulted in approximately equal rates of reinforcement. Each component was associated with a different color keylight. After stability criteria were met, the history-testing condition began: the same keylights were presented as before, but this time a multiple FI FI schedule was in effect. The value of the FI schedules was equal across components, and was equal to the mean interreinforcement interval (IRI) from the last six sessions of history building. Response rates in the history-testing condition following the tandem VI FR condition were higher than those following the tandem VI DRL condition, and these individual response-rate differences persisted for 18 to 31 sessions.

In the experiments cited above, there was a reliable effect of behavioral history on responding in the history-testing condition; however, the extent to which this history effect was a function of the differences in response rates or reinforcement schedules in history-building is not clear. The question remains as to what are the relative contributions of historical response- and reinforcement-rates on subsequent responding in a changed environment.

One approach to disentangling the roles of response rate and reinforcement rate in a history-building condition was undertaken by da Silva, Meginley, and Lattal (2008). In their Experiment 2, da Silva et al. programmed a two-key concurrent schedule such that response rates of pigeons were high for one key (tandem VI FR) and low for the other (tandem VI DRL). Reinforcement rate was approximately equal across components, and response rate was three to six times greater on the tandem VI-FR key than on the tandem VI-DRL key. Differential-reinforcement-of-otherbehavior (DRO) schedules were used to eliminate pecking, and subsequently, extinction (EXT) was introduced to test for resurgence. Differential resurgence occurred across the two components. More responding occurred in the component that previously had generated higher response rates. In their Experiment 3, da Silva et al. used concurrent tandem schedules to program different reinforcement rates on the two response keys, while maintaining approximately equal response rates on both keys. The same pigeons were used as in Experiment 2. Although the experiment that programmed response-rate differences resulted in different amounts of resurgence, the experiment that programmed reinforcement rate differences did not. da Silva et al. concluded that, with other variables held constant, historical response rates were better predictors of resurgence than were historical reinforcement rates.

In another study, a history-building condition similar to those in da Silva et al.'s (2008) Experiment 3 was used to test for differential persistence of history effects (Okouchi \& Lattal, 2006). Okouchi and Lattal used multiple schedules to build parallel operant histories in pigeons. In each of two components, tandem VI DRL schedules were used. For each pigeon the VI and DRL values were adjusted until response rates were similar and reinforcement rates were different between components. For example, for one pigeon, a tandem VI 20-s DRL 4-s schedule in one component resulted in a higher rate of reinforcement than did a tandem VI 120-s DRL 7-s schedule in the other component. For all subjects, the difference in obtained rate of reinforcement across the two components was much greater than the difference in response rates across components. Following history building, history testing was conducted using a multiple FI FI schedule with the same component-correlated stimuli as in the history-building condition. In history testing response rates tended to be higher in the presence of stimuli correlated with the lower rate of reinforcement. Additionally, responding that had been maintained by a higher rate of reinforcement was slightly more resistant to change when the schedule of reinforcement was changed. These effects of reinforcement history, however, were far less robust than were effects of behavioral history in experiments that built parallel histories of divergent response rates and approximately equal reinforcement rates (e.g., da Silva et al., 2008; Freeman \& Lattal, 1992; Metzger, 1992). These data suggest that a history of responding at a high or low rate may result in a more durable effect in subsequent testing with changed contingencies than does a history of higher or lower rates of reinforcement that maintains constant response rates in the two conditions.

Taken together, these studies show that between-component or between-group differences in response rates tend to result in greater and more persistent effects on responding than do differences that result from differences in reinforcement rates (cf. da Silva et al., 2008; Freeman \& Lattal, 1992; Metzger, 1992; Okouchi \& Lattal, 2006; Urbain et al., 1978). Accordingly, it appears that historical response rate is a better predictor of future behavior than is historical reinforcement rate.

Features of an organism's operant history are not the only critical determinants of behavior in subsequent settings. Variables present in the changed-to condition also play a role in determining the effects of operant history. In terms of behavioral history research, history effects are seen most clearly when conditions in the history-testing condition are favorable. Variables in history-testing that have been found to support persistent effects of behavioral history are the schedule of reinforcement (cf. Poling, Krafft, \& Chapman, 1980; Wiener, 1969) and the similarity between the 
history-building and history-testing conditions (cf. Freeman \& Lattal, 1992; LaFrancois \& Metzger, 1993; Metzger, 1992). Wiener (1969) suggested that FI schedules are particularly sensitive to conditioning history. This suggestion has been supported consistently in the behavioral history literature. Behavioral differences engendered in historical conditions have been found to persist longer under FI than VI schedules (cf. Freeman \& Lattal, 1992; Metzger, 1992; Poling et al., 1980). Poling et al. (1980) systematically replicated Urbain et al. (1978). Like Urbain et al., they exposed FR- and DRL-trained groups of rats to a history-testing condition. Instead of using FI schedules during history testing, Poling et al. used VI schedules. As mentioned earlier, high and low response rates persisted for many sessions under Urbain et al.'s FI schedules. In contrast, high and low response rates converged over just a few sessions under the VI schedules employed by Poling et al. This relatively rapid convergence under VI schedules was also shown in Nader and Thompson (1987) in which groups of pigeons were first exposed to 50 or more sessions of FR or DRL schedules. That behavioral history effects are more persistent on FI schedules than VI schedules also has been supported in multiple schedules of reinforcement by Freeman and Lattal (1992) and Metzger (1992). In addition to the schedule of reinforcement in the history-testing condition, stimulus and reinforcement rate similarity between the history-building and history-testing conditions have been shown to be associated with more robust behavioral history effects (cf. Freeman \& Lattal, 1992; LaFrancois \& Metzger, 1993; Metzger, 1992).

\section{Interpretations of History Effects}

\section{Rate of Reinforcing Stimulus Delivery}

Another aspect of the effects of prior history on behavioral persistence has been considered in research on behavioral momentum. The analysis of behavioral momentum originated in the work of Nevin (1974), who argued that response strength was best measured not in terms of the rate of responding, but by the durability or persistence of responding in the face of a potentially disrupting event. Examples of disruptive events include responseindependent presentation of a reinforcing stimulus, pre-session exposure to a reinforcing stimulus, and the implementation of EXT. Nevin borrowed the metaphor of momentum from physics, analogizing the resistance to change of behavior to the resistance to change of a body in motion. In short, based on this metaphor, behavioral momentum theory predicts that higher reinforcement rates will relate positively to resistance to change. The questions at issue in the study of behavioral momentum are the same as in the behavioral history literature: what factors lead to more or less persistent behavior, and what is the relative importance of these factors?

Nevin (1974, Experiment 2) demonstrated stimulus control of response strength. Pigeons were exposed to a multiple VI 2-min VI 6-min schedule of reinforcement. Subsequently, both components changed such that no reinforcement was delivered in either component (multiple EXT EXT), but the same stimuli were presented in each component just as they were in the multiple VI VI. During multiple EXT EXT, responding occurred at a higher rate and was more persistent in the previously VI 2-min component, than in the previously VI 6-min component. Although Nevin (1974) showed greater persistence of responding in the component with a history of a richer reinforcement schedule, this study did not delineate the relative influence of reinforcement and response rate. In Experiment 5 of the same study, Nevin reported that DRL tended to lead to greater resistance to change than did DRH contingencies in the face of disruption by either response-independent food delivery or by extinction. Nevin concluded that although these data suggested that high-rate requirements may result in less resistance to change, this experiment was not designed to test directly this supposition, and response and reinforcement rates were confounded. He indicated that further research would be needed to investigate this unexpected finding.

One way to test the relative importance of reinforcement and response rate is to compare the resistance to change of low-rate behavior maintained by a higher rate of reinforcement with that of high-rate behavior maintained by a lower rate of reinforcement. Nevin, Tota, Torquato, and Shull (1990, Experiment 1) conducted such a test using a two-component multiple schedule. In one component food was delivered according to a VI schedule, and in the other food was delivered according to a concomitant VI variable-time (VT) schedule (i.e., food was in delivered according to a VI schedule, and occasionally the food hopper would operate according to a time-based schedule). Although the rate of food delivery was higher in the component that included response-independent food, response rates were lower in this component. As Lattal (1974) has shown, the addition of response-independent presentations of the reinforcing stimulus tends to slow response rates. When prefeeding and EXT were used to test for resistance to change, responding in the VI VT component was more resistant to change. Nevin et al. (1990) concluded that, in their test, the higher reinforcement rate was more important than higher response rate in determining behavioral persistence. The difficulty with this interpretation, however, is that by increasing reinforcement in this way, the experimenters also decreased response rates in the same component.

Rate of Responding 
Although behavioral momentum theory is well supported, there is some evidence that historical response rate affects the resistance to change of responding, and that lower-rate is more resistant to change than higher-rate responding (cf. Blackman, 1968; Doughty \& Lattal, 2001; Lattal, 1989; Nevin, 1974, Experiment 5; Nevin, Grace, Holland, \& McLean, 2001; Reed \& Doughty, 2005; Tatham, Wanchisen, \& Yasenchack, 1993). Lattal (1989) used multiple tandem schedules to generate different rates of responding while keeping rates of reinforcement approximately equal. In one component higher rates of responding were established with a tandem VI FR 10, and in the other lower rates of responding were established with a tandem VI DRL 5-s schedule. For two of the four pigeons the value of the VI schedule was $300 \mathrm{~s}$ and for the other two pigeons the value was $100 \mathrm{~s}$. The overall rate of reinforcement was approximately equal across the two components. In resistance-to-change tests response-independent food was delivered during intercomponent intervals (ICIs). Lower-rate responding was more resistant to change than was higher-rate responding. Lattal's (1989) experiment was an advancement in disentangling the relative contributions of response rate and reinforcement rate to resistance to change. Although the reinforcement rate was essentially equal across components, different response rates were generated by different contingencies. Under DRL and FR contingencies, the rate of reinforcement is highly dependent upon the rate of responding. Although the rate of reinforcement under VI schedules likewise is dependent on the rate of responding, the rate of responding may vary a great deal under VI schedules without any behaviorally significant change in the rate of reinforcement. A strategy for producing response-rate differences while holding constant the reinforcement rate and the contingencies could be a productive next step in the endeavor to delineate the relative influence of historical reinforcement and response rates on behavior in a novel situation. Such strategies are forwarded in the present set of experiments. Rather than manipulating the reinforcement rate or the contingency on responding, the current study entailed the manipulation of the context in which the contingencies are operative.

\section{Concurrent Schedules and Context}

Preference between schedule-correlated stimuli in concurrent chains is positively related to behavioral persistence. Accordingly, choice between these stimuli and resistance to change are affected similarly by reinforcement history (Grace \& Nevin, 1997; Nevin, 1979). It is possible to influence preference between stimuli, however, without manipulating the contingencies for responding in the presence of those stimuli. Preference, in such a case, is not generated solely by the contingency for responding to the stimulus, but is imposed by the context in which the contingency is imbedded. Belke (1992) developed a procedure to test effects of the context of reinforcement on stimulus preference. He trained pigeons on multiple-concurrent schedules of reinforcement. One component was a concurrent VI 40-s VI 20-s schedule, and the other was a concurrent VI 40-s VI 80-s schedule. Each of the 4 VI schedules was associated with a unique keylight color. In preference-probe trials, the two VI 40-s stimuli were presented concurrently; and in these probes each pigeon responded more on the key with the color that had appeared in the component with the VI 80-s schedule than the key with the color that had previously appeared concurrently with the VI 20-s schedule. Rates of reinforcement on the VI 40-s key in each training component were approximately equal, and therefore historical reinforcement rates for the two VI 40-s stimuli were not likely a factor in the pigeons' differential responding. The differential allocation of responding on test trials was determined to be a function of the history of the context of that test stimulus, and not a function of the history of the rate of reinforcement. Williams and McDevitt (2003, Experiment 1) successfully replicated Belke (1992), and this replication supported the conclusion that preference between two responses can be influenced without adjustment to the contingency operating on those responses.

Although studies have been conducted to examine effects of historical response rate and reinforcement rate on subsequent behavior, none has examined the role of the context in which this reinforcement and responding occur. The training procedures used by Belke (1992) and Williams and McDevitt (2003) served as a starting point in the current set of experiments for the design of history-building conditions that generate different response rates without the use of different reinforcement rates or different contingencies across components.

\section{Statement of the Problem}

Exposure to contingencies of reinforcement influences subsequent behavior. When asked to account for behavior that varies from what is expected, behaviorally oriented researchers and practitioners frequently invoke learning history. Relatively little research has been conducted, however, that delineates historical variables that result in enduring effects on behavior. For example, how do differential response rates and reinforcement rates contribute to future responding? Which is a more important determinant of future responding, response rate, or reinforcement rate? Several researchers investigating this issue have suggested that response rates are the more important determinant (e.g., da Silva et al., 2008; Freeman \& Lattal, 1992; Metzger, 1992; Okouchi \& Lattal, 2006; Urbain et al., 1978). In these studies two different behavioral histories were built, either within or across subjects. Response 
rates differed and reinforcement rates were held constant, or reinforcement rates differed and response rates were held constant. Following history-building, effects of these histories were compared in history-testing conditions.

In previous research that has generated different response rates and held constant reinforcement rates, different contingencies of reinforcement determined the response rates. For example, in most cases, tandem VI FR schedules were used to generate high response rates, and tandem VI DRL schedules were used to generate low response rates. Although approximately equal rates of reinforcement were obtained, it is not clear whether it was the response rates per se or the different contingencies that generated these response rates that were responsible for the effects observed. A strategy for investigating the influence of response rate on subsequent behavior, while holding constant reinforcement rates and reinforcement contingencies is suggested by the work of Belke (1992). Belke showed that responding could be influenced by the context in which the contingencies are imbedded.

Although previous studies have generated response-rate differences by using different contingencies of reinforcement in two conditions or components, the current study was designed to generate these differences by manipulating the context in which the contingencies are operative. Using a multiple-schedule, two different histories were built within each subject. In each of three experiments, one component of the multiple schedule was designed to generate a high response rate, and in the other component was designed to generate a low response rate. The scheduled reinforcement rates for those responses were constant across the two history-building components. The context in which the contingencies of reinforcement operated influenced response rates, and effects of these historical response rate differences were compared in subsequent history-testing conditions. This use of context or alternative sources of reinforcement to control response rates differed from methods typically used in studies of behavioral history. 


\section{Chapter 2: Experiment 1}

In Experiment 1 the same schedules employed by Belke (1992) and McDevitt and Williams (2003), were used in attempt to generate differences in response rate by manipulating the context in which responding occurs. Like previous research designed to study effects of historical high and low response rates on subsequent responding, reinforcement rate was equal between two responses (e.g., Freeman \& Lattal, 1992; Metzger, 1992; Urbain et al., 1978; Weiner 1969). Unlike previous behavioral history research, in Experiment I of the current study only VI schedules were used, and therefore response rate was not directly constrained by the schedule as it would be under DRL, DRH, or ratio schedules. Using VI schedules identical reinforcement rates can be obtained with a wide range of response rates. It is possible that in previous studies the persistence of response-rate differences partly was a function of the features of the reinforcement schedules, rather than of the historical response rates per se. As in previous studies, to the extent that historic response rate influences subsequent response rate under changed contingencies, response-rate differences should persist under those changed contingencies.

\section{Method}

\section{Subjects}

The subjects were 4 experimentally naive male White Carneau pigeons maintained at $80 \%$ of their ad libitum body weight. The pigeons were housed separately in a colony room with continuous access to water and digestive grit.

\section{Apparatus}

Experimental sessions were conducted in four identical sound-attenuating operant conditioning chambers. The chamber work areas were $32 \mathrm{~cm}$ long by $30 \mathrm{~cm}$ high by $30 \mathrm{~cm}$ wide. On an aluminum work panel comprising one wall of each chamber were three $2.5-\mathrm{cm}$ diameter response keys, with the centers approximately $25 \mathrm{~cm}$ from the chamber floor. The middle key was centered on the midline of the work panel and each of the other two keys each was located approximately $9 \mathrm{~cm}$ from the center key (center to center) and $6 \mathrm{~cm}$ from the chamber side wall. The left, center, and right keys were transilluminated by a white, amber, red, or green light as described in the procedure section. Keys were operated by a force of approximately $0.15 \mathrm{~N}$. Reinforcement was 2.5 -s access to mixed grain from a hopper located behind a $4.5-\mathrm{cm}$ square feeder aperture located on the midline of the work panel $9 \mathrm{~cm}$ from the floor. When the hopper was raised into the aperture, the latter was illuminated by a 28 -volt hopper light. A house light, located in the lower right corner of the work panel, was on at all times during the session except during reinforcement and ICIs. Ventilation fans masked extraneous noise. In an adjacent room, an IBM®-compatible personal computer operated MED-PC® hardware programming software which in turn controlled experimental sessions and recorded data.

\section{Procedure}

Preliminary Training. Magazine training was followed by hand-shaping of the key-peck response on of each of the keys. First, response rates were increased using VR schedules with response requirements that were increased across sessions. Next, responding was maintained on a VI schedule that was leaned over several sessions to VI $40 \mathrm{~s}$ in the presence of each the three keys. During VI leaning, each of the keys was presented individually. In each leaning session the illuminated, operative key alternated quasirandomly, with the restriction that no more than two of one key location could occur successively. Keylight colors in preliminary training were the same as those in subsequent sessions. Each key was presented 20 times per session totaling 60 reinforcers per session.

Multiple Schedule. Prior to each experimental session, one of six multiple schedule component sequences was selected quasirandomly with the restriction that no more than two of one type of sequence occurred successively. This algorithm for selecting sequences and components was used in each condition, and all sequences are provided in Appendix A. Each experimental session was composed of a two-component multiple schedule. Components alternated quasirandomly with the following restrictions: a single component type was not repeated more than three times consecutively, and an equal number of each component type was scheduled during each session. Sessions were conducted seven days a week and included 12 to 16 components of each type (the total number of components was decreased for some pigeons from 32 as needed to prevent them from exceeding their running weights). Additionally, each time the component type changed within a session, there was a 5-s ICI. During the ICI the keys were inoperative and all lights in the chamber were extinguished. No ICI preceded components that followed components of the same type. For example, if Component A was followed by Component A, there would be no ICI separating these two components. Distributions for the VI schedules were generated according to the progression described by Fleshler and Hoffman (1962). Each session was preceded by a 90-s blackout, during which 
time all lights were off in the experimental chambers, and all response keys were inoperative. Including the 90-s presession blackout, ICI, and reinforcement time, the session duration ranged from $30 \mathrm{~min}$ to $1 \mathrm{hr}$. A history-building condition that was designed to build response-rate differences was followed by a history-testing condition. Table 1 shows the number of sessions of each condition for each pigeon.

Table 1: Number of Sessions for Each Pigeon in Experiment 1

\begin{tabular}{cccc}
\hline Pigeon & Pretraining & History Building & History Testing \\
\hline 975 & 18 & 71 & 67 \\
685 & 18 & 60 & 77 \\
179 & 17 & 60 & 79 \\
429 & 18 & 73 & 71 \\
\hline
\end{tabular}

History Building. Once magazine training, key-peck shaping, and schedule leaning were complete, Pigeons $975,685,179$, and 429 were exposed to the history-building condition. The history-building condition for this experiment was composed of a multiple concurrent (VI VI) / (VI VI) schedule of reinforcement. Components were $60 \mathrm{~s}$ in duration. The center key was illuminated in each component of history building, along with one of the side keys. The schedules of reinforcement on the center key were designed to engender low response rates on the side key in Component A, and high response rates on the side key in Component B, while maintaining identical schedules of food delivery across the two side keys. It was expected that, similar to the results reported by McDevitt and Williams (2003), response rate on the side key would be lower in the component with a higher reinforcer rate on the center key, and higher in the component with a lower reinforcer rate on the center key.

The upper portion of Figure 1 illustrates the history-building condition at the terminal schedule values. Stimulusposition-schedule combinations for each pigeon are detailed in Table 2. The arrangement for Pigeon 975 provides an example: In Component A, a VI-40-s schedule was effected on the right-green side key, and a richer schedule (VI 20 s) was effected on the amber center key (top row of Figure 1). In Component B, a VI-40-s schedule was effected on the left-red side key, and a leaner schedule (VI $80 \mathrm{~s}$ ) was effected on the white center key (second row of Figure 1). In each component the contingencies arranged on the center key constituted a contextual schedule designed to affect the rate of responding on the side key. 


\section{Experiment 1}

\section{History Building}

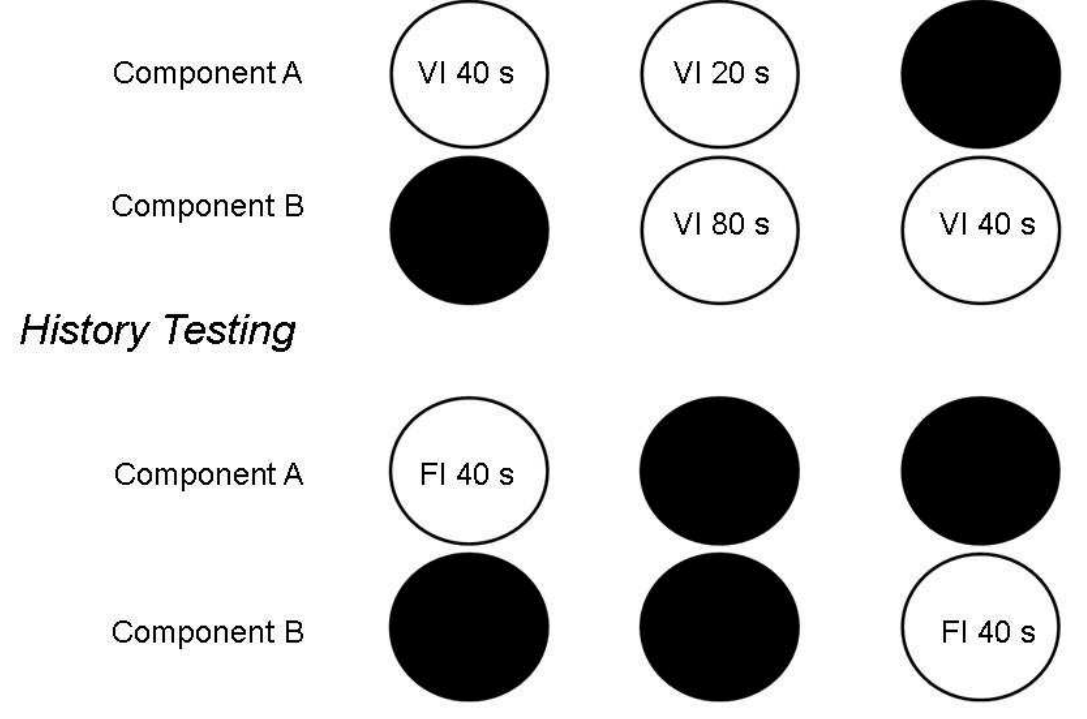

Figure 1: Illustration of history-building and history-testing conditions in Experiment 1.

In each component, a peck-peck changeover delay (COD) was effected on the two keys (Shahan \& Lattal, 1998): when pecking switched from one key to the other, the first peck on the switched-to key began a delay. If no pecks to the other key occur during this delay, reinforcement was available on the switched-to key according to the programmed reinforcement schedule. In the first sessions of history building, VI-schedule and COD values were adjusted to maximize the difference in response rates between the side keys across the two components. Appendix B shows the number of sessions conducted with each pigeon at each set of schedule values. Regardless of adjustment to the schedule values, the scheduled reinforcement rates for the two side keys were equal to one another in each session. The history-building condition did not end for a pigeon until there were at least 20 consecutive sessions at the terminal values (and a minimum total of 60 sessions had occurred).

Table 2: Stimulus-Position-Component Combinations for Each Pigeon in Experiment 1

\begin{tabular}{cccccc}
\hline & \multicolumn{2}{c}{ Component A } & & \multicolumn{2}{c}{ Component B } \\
\cline { 2 - 3 } \cline { 5 - 6 } Pigeon & Side & Center & & Side & Center \\
\hline 975 & Green (Right) & Amber & & Red (Left) & White \\
685 & Green (Left) & Amber & & Red (Right) & White \\
179 & Red (Right) & White & & Green (Left) & Amber \\
429 & Red (Left) & White & & Green (Right) & Amber \\
\hline
\end{tabular}

History Testing. A history-testing condition followed history building. As illustrated in the lower portion of Figure 1, history testing consisted of a two-component multiple FI 40-s FI 40-s schedule of reinforcement, and the stimuli presented on the side keys were identical to those in the history-building condition. In history testing, the center key was never illuminated nor operative, and there was no alternative source of food. For each pigeon, the FI values were equal to the VI values operating on the side keys in history building. In each session, following the presession blackout, two FI components alternated in the same manner as in history building. Each component ended after delivery of the second reinforcer; thus, components were approximately $80 \mathrm{~s}$ in duration. The history-testing condition was conducted: (a) until a minimum of 67 sessions were completed, and (b) for the last 3 (minimum) there 
was a consistent difference in response rate across components (e.g. response rate in Component A was higher for three consecutive days), or (c) for a maximum of 80 sessions.

$$
\text { Results }
$$

\section{History Building}

Response Rate. The following abbreviations refer to response rates on each response key: Pas and Pac refer to the rate of responding on the side key and center key in Component $\mathrm{A}$, and $\mathrm{Pbs}$ and Pbc refer to the rate of responding on the side and center keys in Component B. Three comparisons of responses rates were made: (1) between the center and side key in each component (Pas vs. Pac, and Pbs vs. Pbc), (2) between the two side keys (Pas vs. Pbs) across components, and (3) between the sums of the response rates on the side and center keys [(Pas + $\mathrm{Pac})$ vs. $(\mathrm{Pbs}+\mathrm{Pbc})$; hereafter, overall response rate] across components.

Table 3:Mean (and Range) Response Rate per Min over the Last Twenty Sessions of the History-Building Condition in Experiment 1

\begin{tabular}{|c|c|c|c|c|c|c|}
\hline \multirow[b]{2}{*}{ Pigeon } & \multicolumn{2}{|c|}{ Component A } & \multicolumn{2}{|c|}{ Component B } & \multicolumn{2}{|c|}{ Proportional Response Rate } \\
\hline & Pas (VI40s) & Pac (VI20s) & Pbs (VI40s) & $\mathrm{Pbc}(\mathrm{VI} 80 \mathrm{~s})$ & Side Keys $^{a}$ & Comp. Sums $^{b}$ \\
\hline 975 & $39(22-58)$ & 75 (51-96) & $46(36-62)$ & 28 (19-39) & 0.54 & 0.40 \\
\hline 685 & $37(26-45)$ & $89(68-102)$ & 77 (58-99) & $36(19-50)$ & 0.67 & 0.47 \\
\hline 179 & $24(14-36)$ & $43(35-51)$ & $44(31-55)$ & $20(11-29)$ & 0.65 & 0.49 \\
\hline 429 & $40(24-51)$ & $69(57-78)$ & $41(33-51)$ & $27(15-39)$ & 0.50 & 0.38 \\
\hline
\end{tabular}

${ }^{a}$ Proportional Response Rate (Side Keys) = Side B / (Side B + Side A). ${ }^{b}$ Proportional Response Rate (Component Sums $)=($ Side B + Center B $) /($ Side A + Center A + Side B + Center B $)$.

Table 3 shows the mean and range response rate across the last 20 sessions of history building for each of the four alternatives. As expected, within each component response rates were higher on the key with the higher reinforcer rate. That is, in Component A, Pac (VI 20 s) was greater than Pas (VI 40 s), and in Component B, Pbs (VI 40 s) was greater than Pbc (VI $80 \mathrm{~s}$ ). The upper panel of Figure 2 shows the proportional response- and reinforcement-rate data for all pigeons within components. In Figure 2, proportional response rate within a component is the quotient of the response rate on the richer alternative divided by the overall response rate for that component. For example, in Component A, the Pac (VI $20 \mathrm{~s}$ ) is richer than Pas (VI $40 \mathrm{~s}$ ). The displayed data result from dividing Pac by the sum of Pac and Pas. A value of 0.50 would reflect equal response rates on the two keys in Component $\mathrm{A}(\mathrm{Pas}=\mathrm{Pac})$. A value greater than 0.50 would result from a greater response rate on the center key in Component $\mathrm{A}$ than on the side key in Component A (Pac $>$ Pas). 


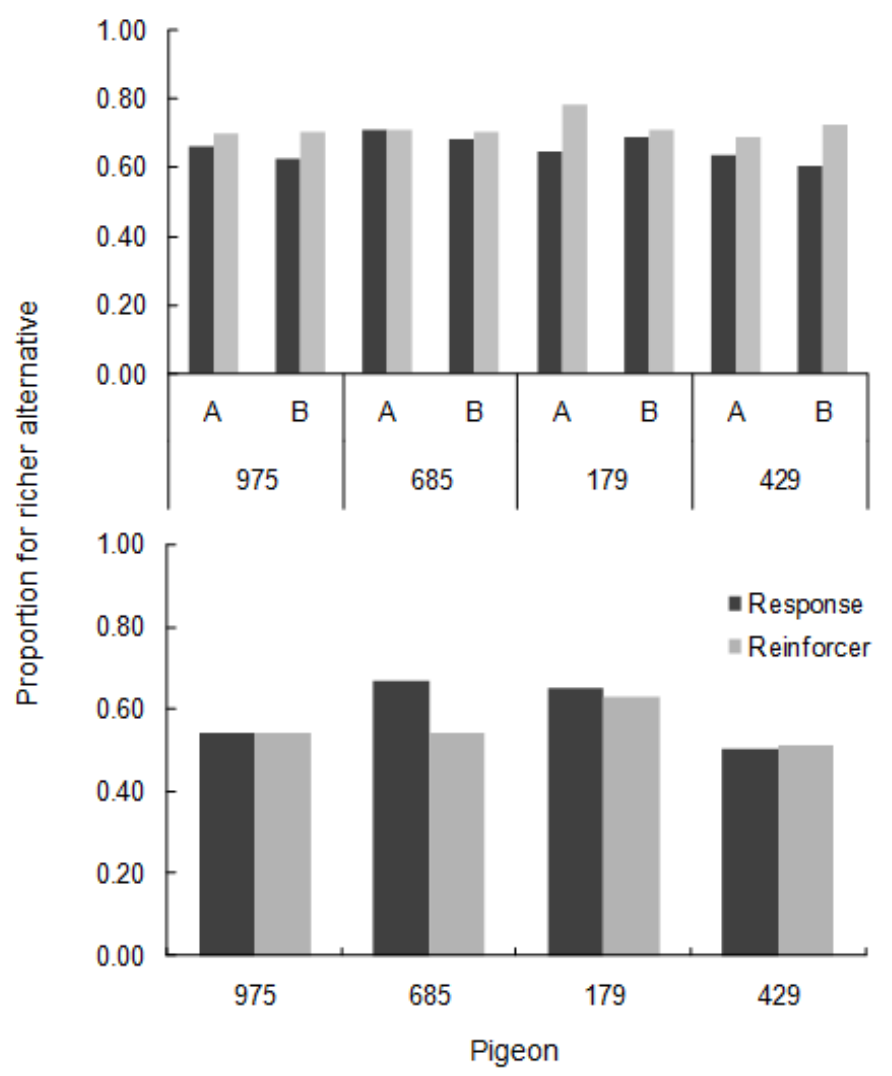

Figure 1: Proportional response rates and reinforcement rates in the history-building condition of Experiment 1. The upper panel shows the quotient of the response rate (dark grey) and reinforcement rate (light grey) on the richer alternative divided by the overall response rate within each component. The lower panel shows the quotient of the response rate (dark grey) and reinforcement rate (light grey) on the side key in Component B divided by the sum of the response rates on the side keys in both components.

Across components, response rate was greater for each pigeon on the side key in Component B than on the side key in Component A (Pbs>Pas). In two pigeons, 975 and 429, the magnitude of the difference in response rates between the two side keys was small, but in Pigeons 685 and $179 \mathrm{Pbs}$ was approximately two times Pas. These differences are summarized in Table 3 as proportional response rates (Side Keys), and are displayed in the lower panel of Figure 2. Proportional response rate is the quotient of Pbs divided by the sum of $\mathrm{Pbs}$ and Pas. A value of 0.50 , therefore, would reflect equal response rates on the side keys across the two components $(\mathrm{Pbs}=\mathrm{Pas})$. A value greater than 0.50 would result from a greater response rate on the side key in Component B than on the side key in Component A (Pbs $>$ Pas).

Finally, overall response rate data are summarized in Table 3 as proportional response rates (Comp. Sums). This value is the quotient of the sum of Pbs and $\mathrm{Pbc}$, divided by the sum of the response rate on each of the four alternatives across the two components. Each pigeon had lower overall response rates in Component B than in Component A. These differences were small in Pigeons 685 and 179 and greater in Pigeons 975 and 429. A small proportional difference was experimentally defined as greater than 0.45 and less than 0.55 .

Reinforcer Rate. Mean reinforcer rates for each alternative across the two components are displayed in Table 4; and proportional within-component reinforcer rate data are displayed in the top panel of Figure 2. Reinforcer rate proportions within components were similar to but generally larger than response-rate differences. Response-rate differences within components, therefore, were less than what would be predicted by the matching law (Herrnstein, 1970). Proportional reinforcer rate data for the side keys across components are displayed in Table 4 and illustrated in the bottom panel of Figure 2. Across components, the rate of reinforcement on the side key in Component B (Rbs) was greater than rate of reinforcement on the side key in Component A (Ras), but in three of the four pigeons, this difference was small. The proportion of Component B side-key reinforcers to all side-key 
reinforcers was largest for Pigeon 179 (0.62). For two pigeons, 685 and 179, response-rate differences exceeded reinforcer rate differences for the two VI 40-s keys, and for the other two pigeons, response-rate differences were close to equal to reinforcer rate differences. Finally, each pigeon had a lower overall rate of reinforcement in Component B than in Component A. For each pigeon, approximately two-thirds of all reinforcers were obtained in Component A. Overall response-rate differences were not as large as would be predicted by the matching law. These data are summarized in rightmost columns of Tables 3 and 4.

Table 4: Mean (and Range) Reinforcement Rate per Hour over the Last Twenty Sessions of the History-Building Condition in Experiment 1

\begin{tabular}{|c|c|c|c|c|c|c|}
\hline \multirow[b]{2}{*}{ Pigeon } & \multicolumn{2}{|c|}{ Component A } & \multicolumn{2}{|c|}{ Component B } & \multicolumn{2}{|c|}{ Proportional SR Rate } \\
\hline & Ras (VI40s) & $\operatorname{Rac}(V I 20 s)$ & Rbs (VI40s) & Rbc (VI80s) & $\begin{array}{l}\text { Side }^{a} \\
\text { Keys }\end{array}$ & $\begin{array}{l}\text { Comp. }{ }^{b} \\
\text { Sums }\end{array}$ \\
\hline 975 & $71(55-85)$ & $163(150-175)$ & $82(70-95)$ & $35(25-45)$ & 0.54 & 0.33 \\
\hline 685 & $67(45-85)$ & $160(130-175)$ & $80(65-98)$ & $34(20-50)$ & 0.54 & 0.33 \\
\hline 179 & $45(30-60)$ & $162(150-180)$ & $77(65-85)$ & $32(20-45)$ & 0.62 & 0.34 \\
\hline 429 & $75(55-90)$ & $164(155-180)$ & $80(65-95)$ & $31(15-45)$ & 0.51 & 0.32 \\
\hline
\end{tabular}

${ }^{a}$ Proportional Reinforcement Rate (Side Keys)= Side B / (Side B + Side A). ${ }^{b}$ Proportional Reinforcement Rate $($ Component Sums $)=($ Side B + Center B $) /($ Side A + Center A + Side B + Center B $)$.

History Testing

Response Rate. Response rate data for each pigeon in Experiment 1 are displayed in Figure 3. Each data point is the mean response rate over a block of 10 sessions (except in pigeons for which the total number of sessions was not a multiple of 10 - in these cases, the last data point is the mean of the last 7 to 11 sessions; see figure captions for details). On the left side of Figure 3, the left portion of each graph displays response rates on the side keys (Pas and Pbs) during the last 20 sessions of history building, and the right portion displays the response rates in history testing (Pas and Pbs). On the right side of Figure 3, the left portion of each graph displays overall response rates in each component $(\mathrm{Pas}+\mathrm{Pac}$ and $\mathrm{Pbs}+\mathrm{Pbc}$ ), and the right portion displays response rates during history testing (for each pigeon, the display of the history-testing data is identical on each side of the figure). In general, compared to response rate on the side keys in the history-building condition, response rates increased initially, and then fell at different rates over the course of the testing condition.

Figure 4 shows the log ratio of response rates in the first session of history testing as a proportion of the mean response rate in the same component from the last 20 sessions of history building. For every pigeon, response rate increased in each component relative to response rate in history building, and this increase was greater in Component A than Component B. This increase over history-building levels in Component A persisted for two pigeons, 975 and 685, for the remainder of the history-testing condition. 

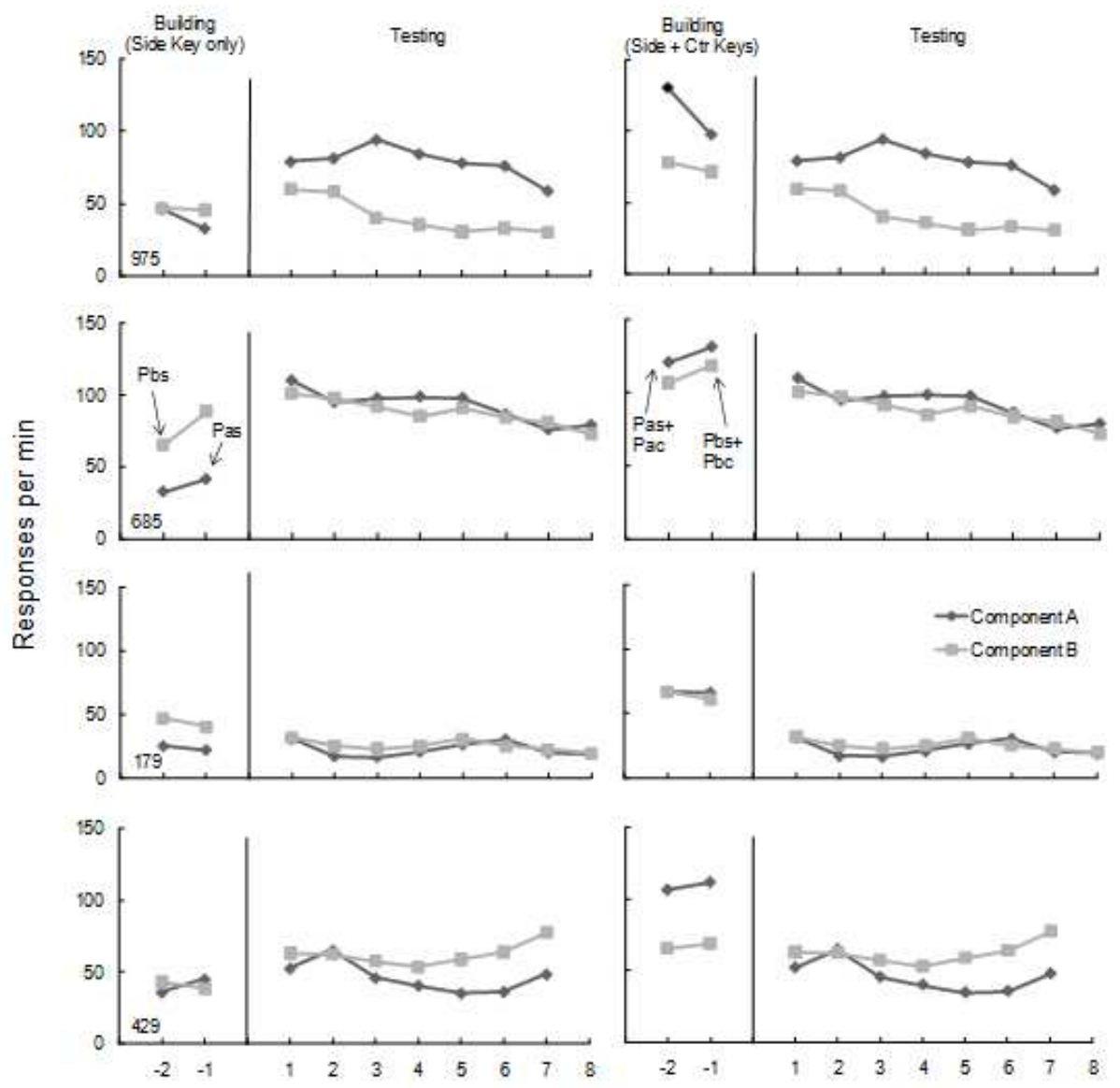

Blocks of 10 Sessions*

Figure 3: Response rate data for each pigeon in Experiment 1. In the left panel, the left portion of each graph displays response rates on the side keys (Pas and Pbs) in the last twenty sessions of history building, and the right portion displays the response rates in history testing (Pas and $\mathrm{Pbs}$ ). In the right panel, the left portion of each graph displays overall response rates in each component $(\mathrm{Pas}+\mathrm{Pac}$ and $\mathrm{Pbs}+\mathrm{Pbc}$ ), and the right portion displays response rates in history testing (for each pigeon, the display of the history-testing data is identical for each side of the figure).

*Each data point represents the mean response rate over a block of 10 sessions, with the following exceptions: For both 975 and 685,the last data point shows the mean of the last 7 sessions, for 179, the last 9 sessions, and for 429, the last 11 sessions. These exceptions apply to all figures for Experiment 1 that display data in 10 -session blocks. 


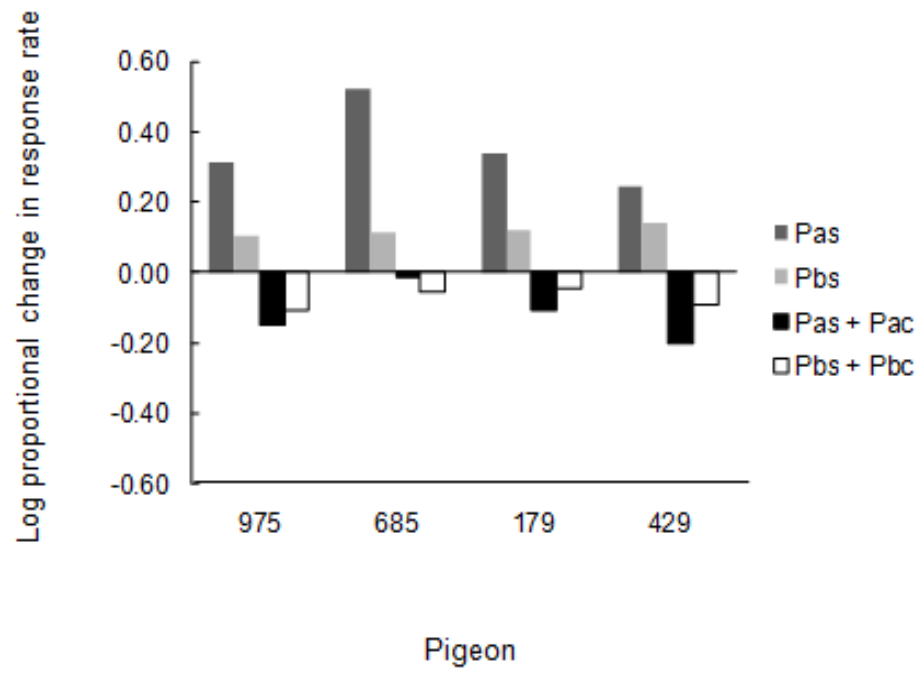

Figure 2: Experiment 1: Log ratio of response rates in the first session of history testing as a proportion of the mean response rate in the same component from the last 20 sessions of history building.

Response-Rate Differences. One index of the persistence of behavioral history is the degree to which differences between responses with different histories persist after each response contacts identical conditions. Figure 5 shows the difference in response rates in Components A and B. On the left portion of each panel are the response rates on the side key in Component $\mathrm{B}$ as a proportion of side key responding in both components [Pbs / (Pbs + Pas); closed diamonds] and the response rates for overall responding in Component $\mathrm{B}$ as a proportion of all responding in both components $[(\mathrm{Pbs}+\mathrm{Pbc}) /(\mathrm{Pbs}+\mathrm{Pbc}+\mathrm{Pas}+\mathrm{Pac})$; open diamonds]. On the right portion of each panel is the proportion of responding in Component $\mathrm{B}$ in history testing. Values greater than 0.50 indicate that the response rate was greater in Component B than in Component A. Differences in response rates across components were not consistent across pigeons. In Pigeons 429 and 179, response rate tended to be greater in Component B, and in Pigeons 975 and 685, response rate was greater in Component A (though in Pigeon 685 this difference is small). For Pigeons 975 and 685, response-rate differences in history testing were more similar to differences in overall response rates (open diamonds) in history building than to the differences in side-key response rates. 


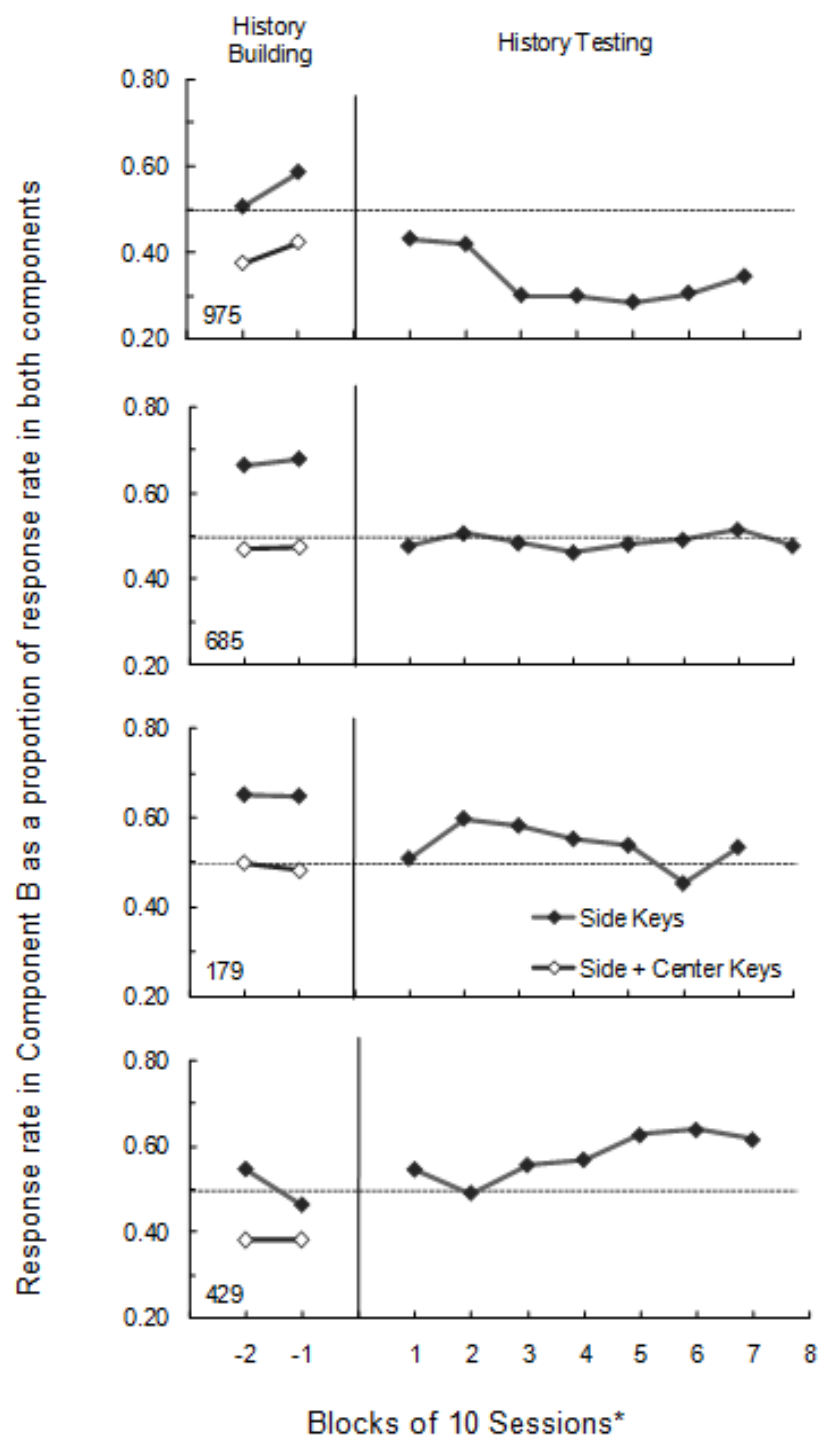

Figure 5: Experiment 1: difference in response rates in Components A and B. In the left portion of each panel are the response rates on the side key in Component B as a proportion of side key responding in both components [Pbs / (Pbs + Pas); closed diamonds] and the response rates for overall responding in Component $\mathrm{B}$ as a proportion of all responding in both components $[(\mathrm{Pbs}+$ $\mathrm{Pbc}) /(\mathrm{Pbs}+\mathrm{Pbc}+\mathrm{Pas}+\mathrm{Pac})$; open diamonds]. On the right portion of each panel is the proportion of responding in Component $\mathrm{B}[\mathrm{Pbs} /(\mathrm{Pbs}+\mathrm{Pas})]$ in history testing.

Rate of Change. Resistance of a response to change in the face of changed environmental events or contingencies commonly is taken as the best measure of the strength of that response (Nevin, 1974; Nevin \& Grace, 2000). In analyzing the persistence of control by previous conditions, therefore, the behavioral momentum literature would suggest an analysis not of the absolute response rates or the differences between these, but of the rate of change of each response, relative to its own baseline rate. When response rate data are plotted as a proportion of their respective baseline rates, the slope of the resulting function can be used as a measure of the rate of change. Shallower slopes result from smaller changes from baseline conditions, and reflect stronger control by the previous contingencies. Additionally, these ratios are commonly expressed as logarithms of proportions, so that equal proportional changes result in equal differences. In this way, response rate changes between low-rate responding can be directly compared with response rate changes between high-rate responding (Nevin \& Grace, 2000). 
Figure 6 displays the log proportion of the response rate in each component of history testing. The proportion of response rates in history testing to side-key response rates in history building is displayed on the left side of the figure, and the proportion of response rates in testing to overall response rates is displayed on the right side of the figure. Side-key proportions were calculated for each component by dividing response rate in history testing by the mean response rate on the side key in the final 20 sessions of history building, and then determining the log of this ratio $\left[\log \left(\mathrm{Pas}_{\text {test }} / \mathrm{Pas}_{\text {build }}\right)\right.$ for Component $\mathrm{A}$, and $\log \left(\mathrm{Pbs}_{\text {test }} / \mathrm{Pbs}_{\text {build }}\right)$ for Component B]. Overall-response-rate proportions were calculated for each component by dividing response rate in testing by mean of the overall response rate in the final 20 sessions of history building $\left[\log \left(\mathrm{Pas}_{\text {test }} /\left(\mathrm{Pas}_{\text {build }}+\mathrm{Pac}_{\text {build }}\right)\right.\right.$ for Component $\mathrm{A}$, and $\log \left(\mathrm{Pbs}_{\mathrm{test}} /\right.$ $\left(\mathrm{Pbs}_{\text {build }}+\mathrm{Pbc}_{\text {build }}\right)$ for Component $\left.\mathrm{B}\right]$. As with response-rate, log-proportion data are presented in 10-session blocks. The slope of each line illustrates the degree to which response rate changed over the course of history testing. The level of the data, with respect to the ordinate, indicates the degree to which response rate is greater or less than it was in history building. A result of "0" on the ordinate would occur were response rate in history testing equal to response rate for the same component in history testing (i.e., equal response rates would yield a ratio of 1 , and the $\log$ of 1 is 0 ). The difference between the left and right sides of the figure is only in the level of the behavior. In the case that the magnitudes of the change in response rates were equal for Components A and B, the levels of the logproportion functions would be the same; and in the case that the rates of change were equal, the slopes would be the same.

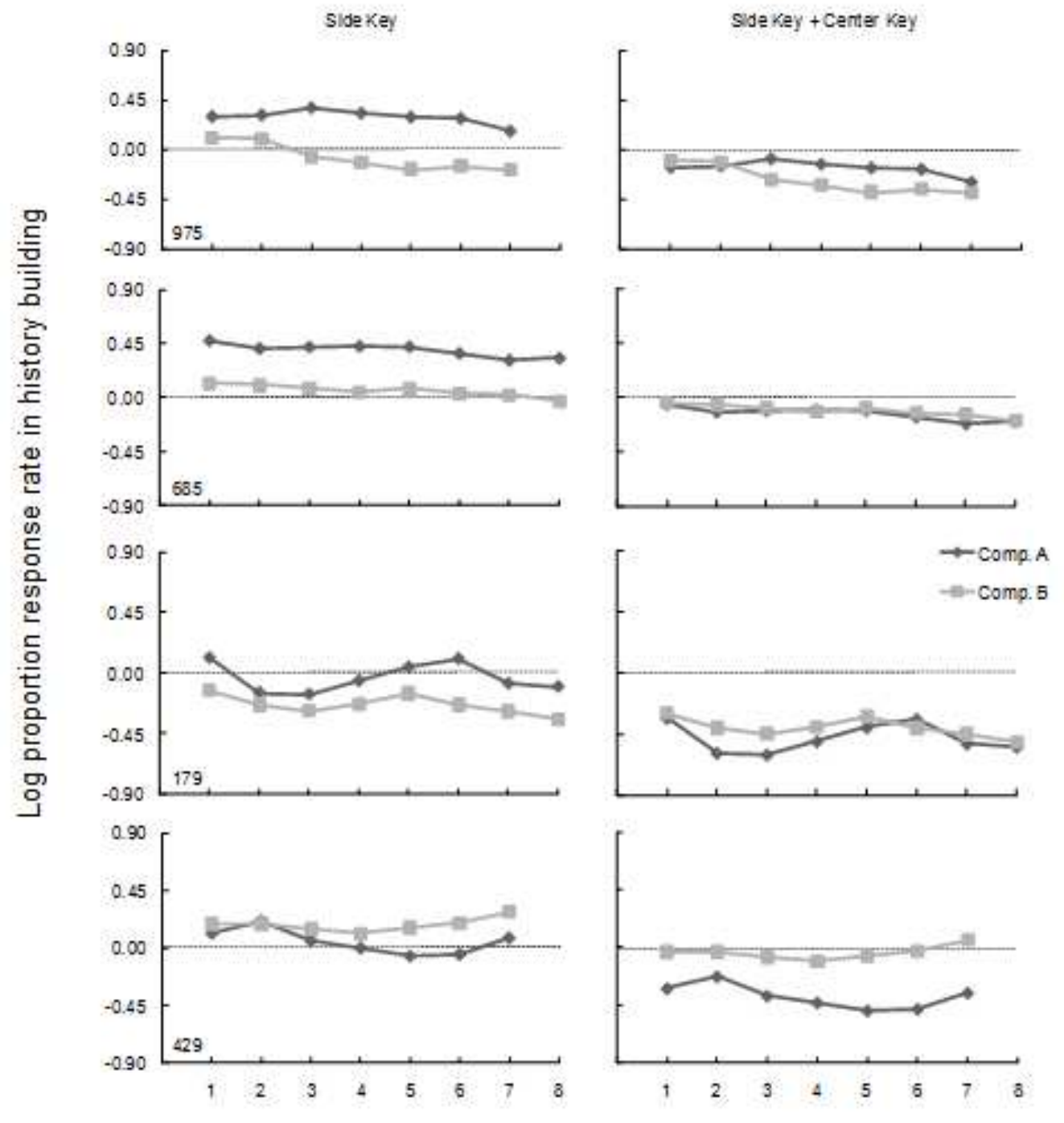

Blocks of 10 Sessions ${ }^{*}$ 
Figure 3: Experiment 1: $\log$ proportion of the response rate in each component of history testing. The proportion of response rates in history testing to side-key response rates in history building is displayed on the left side of the figure, and the proportion of response rates in testing to overall response rates is displayed on the right side of the figure. Side-key proportions are calculated for each component by dividing response rate in history testing by the mean response rate on the side key in the final 20 sessions of history building, and then taking the $\log$ of this ratio $\left[\log \left(\mathrm{Pas}_{\text {test }} /\right.\right.$ $\left.\mathrm{Pas}_{\text {build }}\right)$ for Component $\mathrm{A}$, and $\log \left(\mathrm{Pbs}_{\text {test }} / \mathrm{Pbs}_{\text {build }}\right)$ for Component $\left.\mathrm{B}\right]$. Overall-response-rate proportions are calculated for each component by dividing response rate in testing by mean of the overall response rate in the final 20 sessions of history building $\left[\log \left(\mathrm{Pas}_{\text {test }} /\left(\mathrm{Pas}_{\text {build }}+\mathrm{Pac}_{\text {build }}\right)\right.\right.$ for Component $\mathrm{A}$, and $\log \left(\mathrm{Pbs}_{\text {test }} /\left(\mathrm{Pbs}_{\text {build }}+\mathrm{Pbc}_{\text {build }}\right)\right.$ for Component $\left.\mathrm{B}\right]$. Log-proportion data are presented in 10-session blocks.

Figures 7 and 8 allow a comparison of the rate of change in response rate in the two components. Most of the change in response rate in history testing occurred in the first 20 sessions of that condition. Figure 7 shows the slope of the $\log$ ratios of response rates on the side keys for each pigeon in the first 20 sessions of testing (left panel) and for the all of the test sessions (right panel). To calculate these slopes, the mean of the last 20 sessions of history building was included as the first data point, and individual session data were used for the history-testing condition. In the first 20 sessions, the slopes changed more in Component A than in Component B. For Pigeons 179 and 429, and Component A in 975, the slopes of the log ratio response rate functions were steeper over the first 20 sessions than over the entire course of the testing condition. Session-by-session response-rate data for the first 20 sessions are displayed in Figure 8.

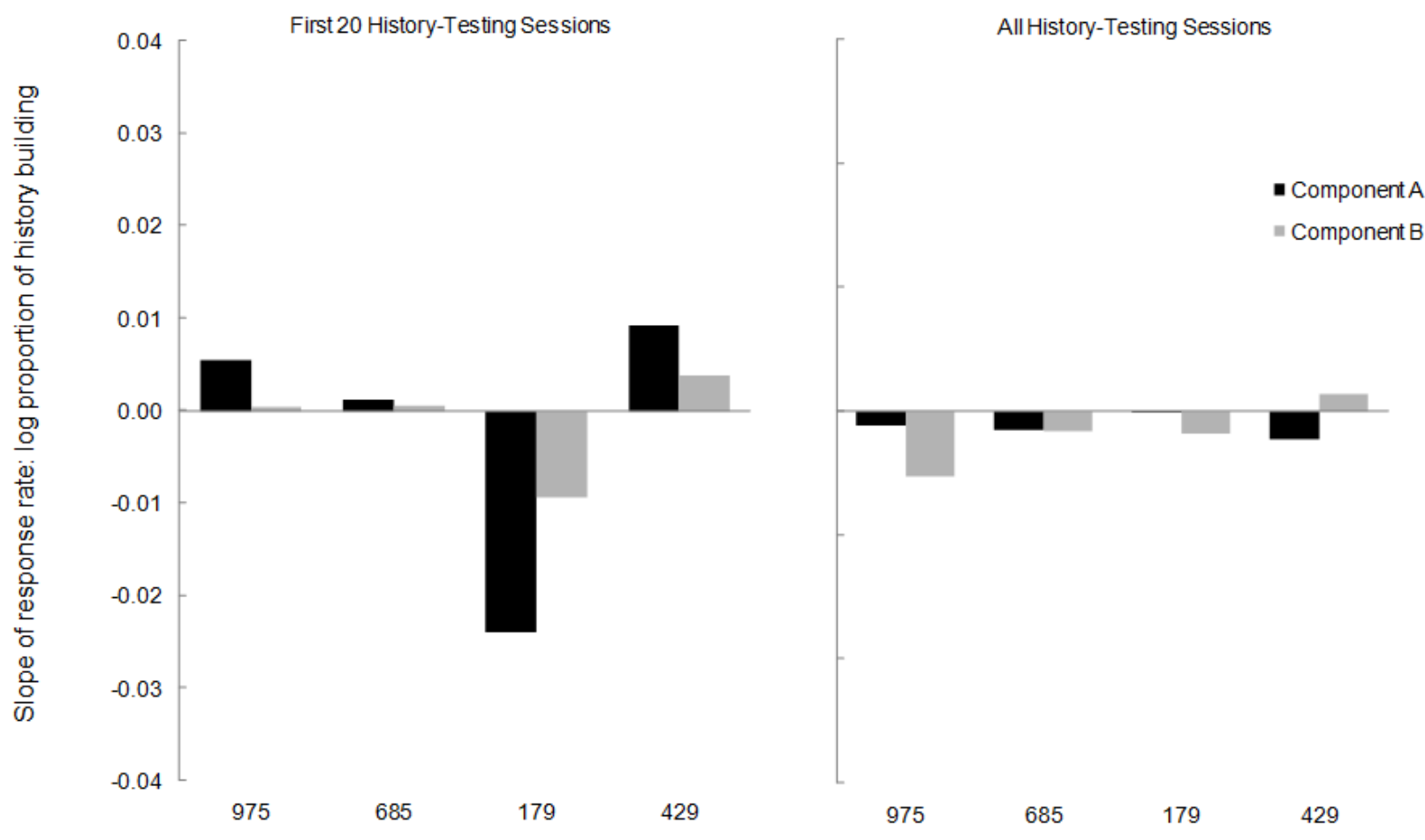

Pigeon

Figure 4: Experiment 1: slope of the log ratios of response rates on the side keys for each pigeon in the first 20 sessions of testing (left panel) and for the all of the test sessions (right panel). To calculate these slopes, the mean of the last 20 sessions of history building was included as the first data point, and individual session data were used for the history-testing condition. 


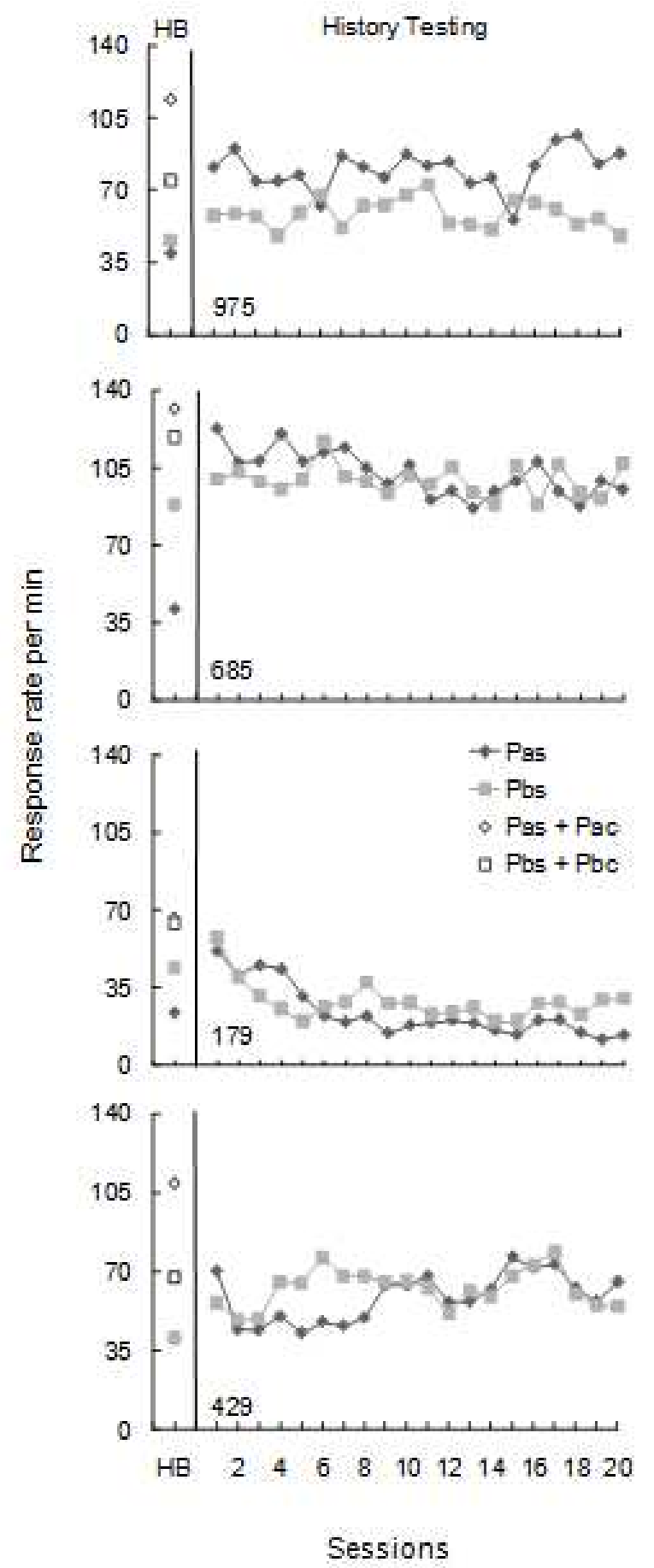

Figure 5: Experiment 1: session-by-session response-rate data for the first 20 sessions.

Pattern of Responding. Another way in which effects of behavioral history may be observed is in the time until the changed-to schedule affects the pattern of responding. The quarter life (QL) is a commonly used measure of the characteristic pattern of accelerated responding over the course of intervals in an FI schedule. The QL represents the proportion of the interval that has passed when $25 \%$ of the responses in the interval have occurred. A QL of 0.25 indicates a linear pattern, and a QL greater than 0.25 indicates a positively accelerated pattern of responding. To calculate the QL for each session, the responses that occurred within each FI interval were sorted 1-s bins. The number of responses in each bin was summed across the session for each component, and the ordinal number of the 
bin which contained the $25^{\text {th }}$ percentile was divided by the total number of bins per interval. As can be seen in Figure 9, for each pigeon, positively accelerated responding developed in both components, and the mean QL for the first 10 sessions was greater in Component A than B (though only slightly so in Pigeons 975 and 685). In both components the QL continued to increase after these first sessions. In each of the four pigeons the maximum 10session mean QL in Component B was greater than or equal to that in Component A.
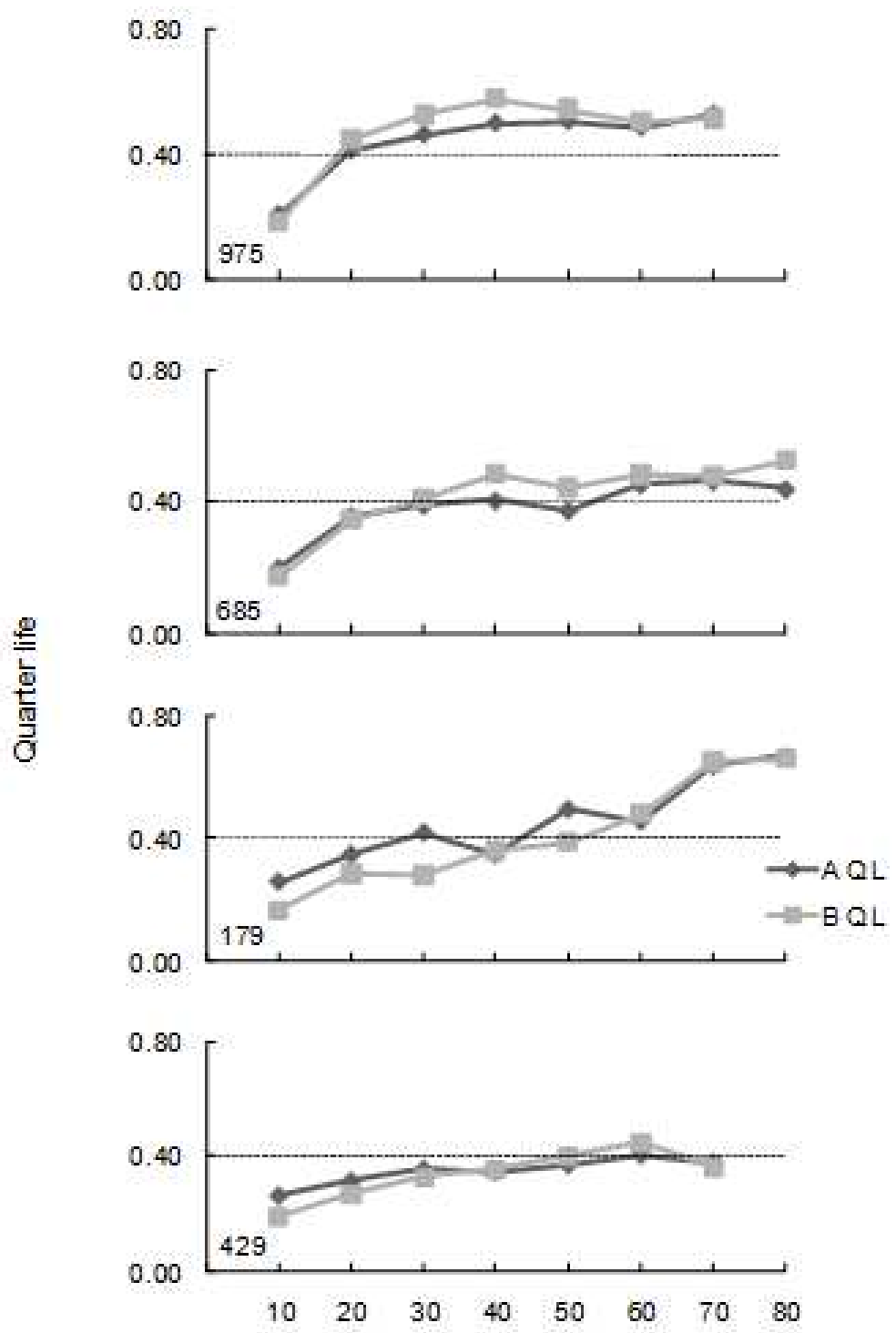

\section{Blocks of 10 Sessions*}

Figure 6: Experiment 1: mean QL in each component of the history-testing condition in 10-session blocks.

Other Measures of Persistence. Table 5 displays several measures of persistence that allow comparison of features of responding between the components in the history-testing condition. The first row of the table shows the number of test sessions in which the response rate was greater in Component B than Component A. The next two rows, sign change 1 and 2 , show the session number at which there was a reversal in the response rates. For example, if Pbs were greater than Pas in history building, and then on the first session of history testing, Pbs were less than Pas, this would constitute a sign change. The next two rows display two measures of convergence of response rates. The $10 \mathrm{resp} / \mathrm{min}$ measure is the session at which the difference between Pas and Pbs had been 10 
responses per minute or less, for five consecutive sessions. Similarly, the $10 \%$ Range measure is the session at which the difference between response rates had been $10 \%$ or less, for five consecutive sessions.

The next eight rows of Table 5 display measures of stability. The measures 10\% Terminal A and 10\% Terminal B show the session at which the response rate had been within $10 \%$ of the terminal response rate for that component (averaged over the last 10 sessions) for 5 consecutive sessions. The next two rows display the first and second times stability criteria were met. The data displayed are the session numbers at which the response rate data met stability criteria, and the number of sessions the data continued to meet stability criteria. Stability was calculated using a 6session window whereby the mean of the first three and the mean of the last three of these sessions were compared to the mean of all six sessions. For response rates to be considered stable, the two means could differ from the mean of all six by no more than 5\%, and this criterion had to be met in both components. Standard deviations were considered for the next four measures. The standard deviation of response rates was calculated in 10-session blocks for each component. Displayed in Table 5 are the standard deviations in Components A and B for the first 10 sessions, and the slope of the change of 10-session blocked standard deviations over the course of the history-testing condition.

Finally, measures of change in the pattern of responding are shown in the last four rows of Table 5. The first session in which the QL is at least 0.40 in each component is shown for each pigeon, as well as the session at which the QL is at least 0.40 for five consecutive sessions. The value 0.40 was selected as a point greater than 0.25 at which responding could be compared across components.

In summary, Table 5 allows a comparison across pigeons of several different measures of persistence in history testing. Although there is inconsistency between pigeons on the number of sessions for which the response rate in Component $\mathrm{B}$ is greater than that in Component $\mathrm{A}$ ( $\mathrm{B}>\mathrm{A} /$ total test sessions), and number of sessions to convergence of response rate between the two components (10 Resp / min and 10\% Range), several measures were more consistent among pigeons. In the last sessions of history building, each pigeon responded at a higher rate on the side key in Component B than A. A value of one or two for the measure Sign change 1 denotes that either in the first or second session, response rate was greater in Component A than B. This was the case for each of the four pigeons. Another measure for which results were consistent across pigeons was the $10 \%$ terminal measure of convergence. Response rates did not reach this criterion in either component for any pigeon in fewer than 68 sessions. Finally, in three of the four pigeons, it took fewer sessions for QLs to increase to at least 0.40 for at least five consecutive days in Component B than A. 
Table 5:Measures of Persistence for Experiment 1

\begin{tabular}{|c|c|c|c|c|}
\hline \multirow[b]{2}{*}{ Measure of Persistence } & \multicolumn{4}{|c|}{ Subject } \\
\hline & 975 & 685 & 179 & 429 \\
\hline $\mathrm{B}>\mathrm{A} /$ total test sessions & $2 / 67$ & $25 / 77$ & $55 / 79$ & $62 / 71$ \\
\hline Sign change 1 & 1 & 1 & 2 & 2 \\
\hline Sign change 2 & 6 & 6 & 6 & 11 \\
\hline $10 \mathrm{Resp} / \mathrm{min}$ & $>67$ & 25 & 15 & 13 \\
\hline $10 \%$ Range & $>67$ & 9 & 33 & 13 \\
\hline $10 \%$ terminal: $\mathrm{A}$ & $>67$ & 71 & $>79$ & $>71$ \\
\hline $10 \%$ terminal: $\mathrm{B}$ & $>67$ & 73 & $>79$ & 68 \\
\hline 1st Stable (no. sessions) & $7(2)$ & $6(7)$ & $17(1)$ & $15(1)$ \\
\hline 2nd Stable (no. sessions) & $10(1)$ & $14(2)$ & $38(2)$ & $19(1)$ \\
\hline SD: A (1st 10 sessions) & 8.10 & 7.40 & 13.36 & 9.89 \\
\hline SD: B (1st 10 sessions) & 6.20 & 6.38 & 10.56 & 8.86 \\
\hline $\begin{array}{l}\text { Slope of change in SD: A } \\
\text { (10 session blocks) }\end{array}$ & -0.15 & 0.12 & -0.77 & -0.33 \\
\hline $\begin{array}{l}\text { Slope of change in SD: B } \\
\text { (10 session blocks) }\end{array}$ & -0.44 & -0.04 & -0.31 & -0.11 \\
\hline $\begin{array}{l}\mathrm{QL}>=0.40: \mathrm{A} \\
(1 \mathrm{st} \text { instance })\end{array}$ & 12 & 17 & 9 & 24 \\
\hline $\begin{array}{l}\mathrm{QL}>=0.40: \mathrm{B} \\
(1 \mathrm{st} \text { instance })\end{array}$ & 12 & 16 & 15 & 29 \\
\hline $\begin{array}{l}\mathrm{QL}>=0.40: \mathrm{A} \\
(5 \text { consec days })\end{array}$ & 20 & 33 & 26 & 55 \\
\hline $\begin{array}{l}\mathrm{QL}>=0.40: \mathrm{B} \\
(5 \text { conseq days })\end{array}$ & 16 & 32 & 56 & 48 \\
\hline
\end{tabular}

\section{Discussion}

In many studies of behavioral history effects, reinforcement rates are held constant across two responses while response rates vary as a function of some feature of the reinforcement schedule that exerts strong influence over 
response rate (i.e., pacing contingencies such as DRH or DRL, or ratio schedules). In contrast, in the present procedure reinforcement rates were held constant across two responses while responding was affected by alternative sources of reinforcement within the same context as the response. Similar to Freeman and Lattal (1992) different histories each were built within sessions of a single history-building condition, as separate components of a twocomponent multiple schedule.

In the current study effects of behavioral history were indicated by 1) between-component response-rate differences of greater than $10 \%$ that persisted for 9, 13, 33 and $>67$ sessions, in Pigeons 685, 429, 179, and 975, respectively; 2) an initially greater rate of change in response rate in Component A than Component B in all pigeons; 3) betweencomponent differences in rate of change of response rate over the course of history testing in three of the four pigeons; 4) failure of response rates in both components to be within $10 \%$ of terminal response rates for the respective component within the first 67 sessions in all pigeons; and 5) a greater rate of increase in QL in Component A than B over the first 10 sessions in all pigeons.

Although the history effects that occurred in this study varied across pigeons, the historical differences between the contexts of the two components had some lasting effects on responding. The primary purpose of this experiment, however, was to investigate effects of historically different rates of responding generated by equal schedules of reinforcement. As shown in Appendix B, in an attempt to achieve response-rate differences similar to those reported by McDevitt and Williams (2003), adjustments were made to the schedules of reinforcement and CODs in the history-building condition. Response-rate differences in history building were small in two pigeons, but these small differences were consistent with several experiments that have employed training conditions similar to the historybuilding condition in this experiment (see Table 6). In these experiments, when the two common-schedule keys were presented together in preference-probe trials, pigeons responded more to the stimulus that previously had been presented concurrently with a relatively lean alternative (Belke, 1992; Bell \& Williams, 2002; McDevitt \& Williams, 2003, Experiment 1, part 1; Williams \& Bell, 1996, Experiment 1). In the current study, response-rate differences between the common-schedule side keys in history building were not consistent for all pigeons, and therefore there was no clear way to evaluate the relative effect of response vs. reinforcement variables on subsequent responding. In light of this issue the question became, would the results of history testing be similar to results found in preference-probe studies that employed similar conditioning schedules to those in the current experiment? Given the preference shown in these studies for the side key that had been relatively rich in the multiple concurrent schedule, and the demonstrated correlation between preference and resistance to change (Nevin, 1979, 1992; Nevin \& Grace, 2000), it was reasonable to ask whether the historical contextual differences of higher or lower rates of reinforcement on an alternative response key in the two components would have different and persistent effects on responding when the contingencies in both components were changed to the same FI schedule of reinforcement. Results consistent with previous studies that have used similar schedule arrangements would be obtained if a higher rate of responding were to occur in Component $\mathrm{B}$ (with the stimulus that was relatively rich in history building) than Component A (with the stimulus that was relatively lean in history building).

Although responding in history building was similar to responding in most previous studies that used similar schedules, results from history testing were different from preference-probe results from the same studies (e.g. Belke, 1992; Bell \& Williams, 2002; McDevitt \& Williams, 2003; Williams \& Bell, 1996). In these studies, in concurrent-operant preference probes pigeons responded more to the stimulus that had been presented previously in the context of a relatively lean alternative (Component B, in Experiment 1 of the current study). In contrast, in the first testing session of the current study three of the four pigeons responded at a higher rate in the presence of the stimulus that had been presented previously in the context of the relatively rich alternative (Component A). In sum, contrary to findings from preference-probe studies, the historical contrast between concurrent schedules of reinforcement within the components of the multiple schedule did not lead to reliably greater response rates on the historically relative-rich common-schedule key $(\mathrm{Pbs})$.

The pattern of higher response rates in Component A persisted throughout the experiment for one pigeon (975). For another pigeon (685) although the differences were small and there were some periods of reversal, response rates tended to be higher in Component A than B throughout the experiment. For pigeons 179 and 429, the initial increase in response rate on the common-schedule keys was not as dramatic as in 975 and 685, and response rate in

Component B consistently exceeded that in Component A in most of the remaining sessions (Pigeon 179: 54/78, and Pigeon 429: 62/71 sessions). It is likely that that these differences from results of preference-probe tests in the literature are due to differences in the testing procedure.

Perhaps the apparently inconsistent finding of response-rate differences on the side keys in the testing condition in this study resulted from differences in the determinants of response rate in concurrent vs. multiple schedules. In the 
concurrent preference-probe procedure, both side keys are available, and in the multiple schedule test procedure (in the current study) only one key is available. The availability of two keys may have allowed different features of behavioral history to influence responding. For example, in history building, the behavior controlled by the contingencies operating in each component in the multiple concurrent schedule key may have included not only responding to, but also responding away from each key. As several have argued (Gibbon, 1995; Grace \& Savastano, 1997; McDevitt \& Bell, 2008; Nevin \& Grace, 2000; Zentall, Weaver, \& Sherburne, 1996; Williams \& Bell, 1996, Experiment 2), the key that was previously relatively rich may be pecked more in the preference probe because it was relatively rich in its component, and therefore the pigeon was likely to respond on that key, or it may be pecked more because the other key was relatively lean, and therefore the pigeon was likely to respond away from that other key. Along these lines, in the history-testing condition for the current procedure, if there were a tendency to respond away from the previous relatively lean key (the side key in Component A), there would have been no other key to peck. Perhaps the increase in response rates on the side key in Component A in history testing was in part a result of a reallocation of former center key responding to the side key (McLean, 1992; Hinson \& Staddon, 1978). These theories will be discussed further in the General Discussion. 


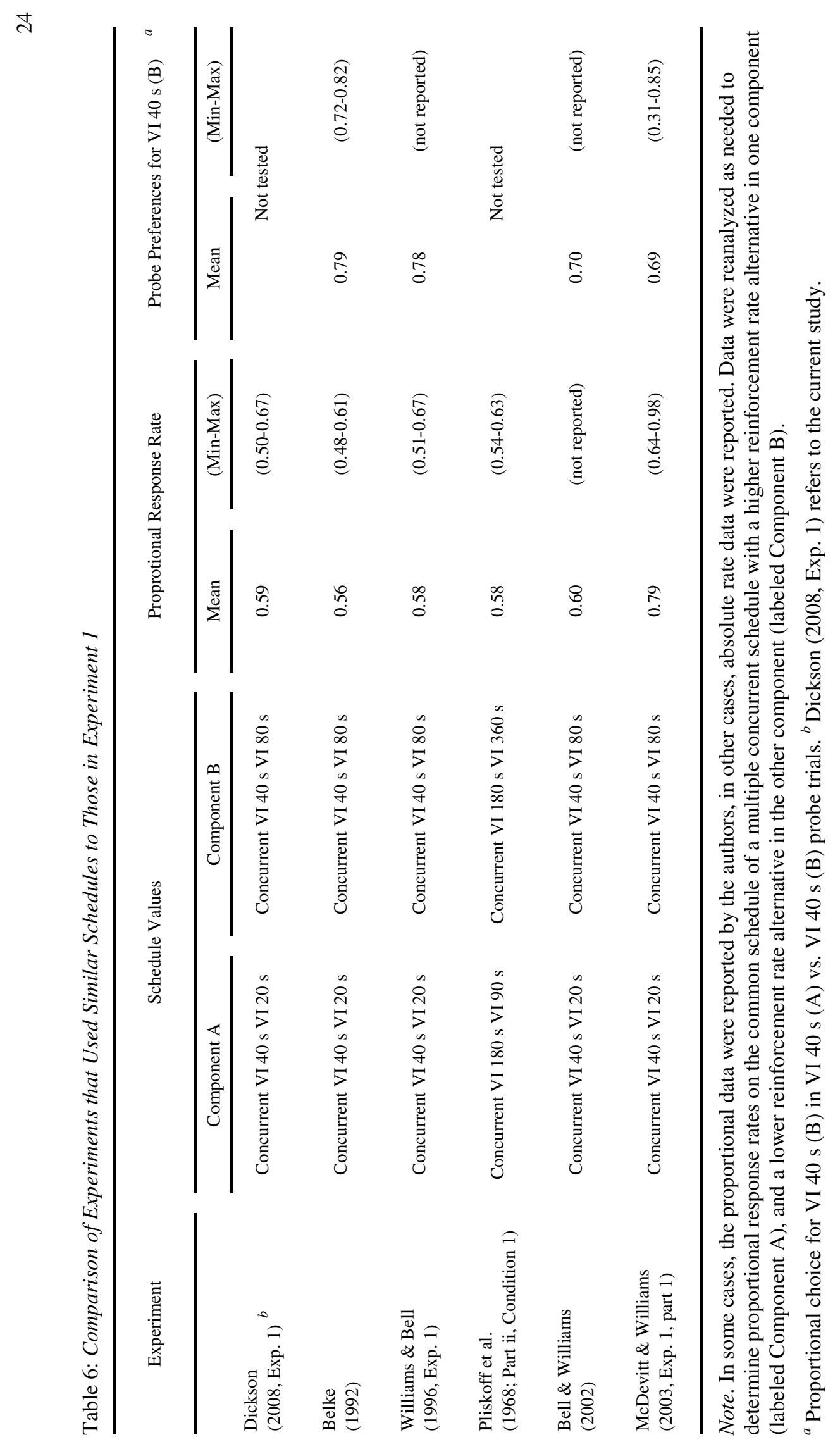


The original question of the degree to which historical response rate vs. reinforcement rate exerts persistent control over subsequent responding is best addressed in this experiment with an analysis of the response rates of Pigeons 685 and 179. These are the two pigeons with the largest response-rate differences in the history-building condition. Interestingly, these are also the two pigeons with the smallest response-rate differences in the history-testing condition. In the case of Pigeon 685, response rates tended to be greater in Component A, and in the case of Pigeon 179 , response rates tended to be greater in Component B. Although the response-rate differences on the side keys from history-building did not persist into the history-testing condition (or increase, as was the case in the concurrent operant preference studies of Belke, 1992; and others), it is not clear why they did not persist. Because responding in history testing was so different from that in history building, the cause of differences in responding between the two components in history testing was unclear - was responding in history testing a function only of the reinforcement schedules operating in that condition, or did some feature of behavioral history affect responding in an unspecified way? Although some of these effects did not occur in the direction predicted by either the preference-probe literature or literature that indicates low-rate responding to be more resistant to change, there is evidence that behavioral history did result in some persistent effects on responding in the history-testing condition (most notably, perhaps, the is lack of convergence to terminal response rates for each pigeon).

Despite the use of almost identical procedures, the response-rate differences obtained by Williams and McDevitt (2003) were not obtained in history building in Experiment 1. In Experiment 2 the problem of generating responserate differences was attacked more directly by employing two different rate-generating contingencies in the contextual schedule in each component. 


\section{Chapter 3: Experiment 2}

The contextual contingencies in Experiment 1 did not result in the consistent response-rate differences earlier reported by McDevitt and Williams (2003), using a similar procedure. In Experiment 2 there was attempt again to establish a history of high- and low-rate responding on two response keys with equal rates of reinforcement. As in Experiment 1, the history-building condition consisted of multiple-concurrent schedules whereby the scheduled reinforcement rate was equal between the two side-key responses, and contextual differences were provided by an alternative source of reinforcement on a second center key in each component. In Experiment 1, reinforcement schedules on each key were VI schedules, and therefore they did not control directly response rate for the side-key or alternative responses. In Experiment 2, reinforcement schedules on the common-schedule side keys were VI schedules, but on the center keys, were rate-controlling schedules. Again, as in Experiment 1, to the extent that historical response rate influences response rate under changed contingencies, response-rate differences should persist under those changed contingencies, even when those responses historically have been reinforced on identical schedules.

\section{Method}

\section{Subjects and Apparatus}

The subjects were four experienced male White Carneau pigeons maintained at $80 \%$ of their ad libitum body weight. The pigeons were housed separately in a colony room with continuous access to water and digestive grit. These were not the same pigeons as in Experiment 1. The apparatus was identical to that used in Experiment 1.

\section{Procedure}

Preliminary Training. Preliminary training sessions for Experiment 2 were identical to those for Experiment 1.

Multiple Schedule. Many procedural details of the multiple schedule sessions in Experiment 2 were the same as in Experiment 1. These identical features include the number of sequences and components, the frequency and duration of sessions, and the presession blackout, the ICI, and the VI schedule distributions. As in Experiment 1, a history-building condition that was designed to build response-rate differences was followed by a history-testing condition. Table 7 shows the number of sessions of each condition for each pigeon.

Table 7: Number of Sessions for Each Pigeon in Experiment 2

\begin{tabular}{cccc}
\hline Pigeon & Pretraining & History Building & History Testing \\
\hline 152 & 30 & 57 & 70 \\
236 & 4 & 62 & 71 \\
965 & 3 & 61 & 71 \\
191 & 12 & 64 & 70 \\
\hline
\end{tabular}

History Building. Once magazine training, key-peck shaping, and schedule leaning were complete, Pigeons 152, 236, 965, and 191 were exposed to the history-building condition. The history-building condition for this experiment was a multiple concurrent (VI - Tandem VI FR) / (VI - Tandem VI DRL) schedule of reinforcement. As in Experiment 1, components were $60 \mathrm{~s}$ in duration. The center key was illuminated in each component of history building, along with one of the side keys. The schedules of reinforcement on the center key were designed to engender low response rates on the side key in Component $\mathrm{A}$, and high response rates on the side key in Component $\mathrm{B}$, while maintaining identical schedules of food delivery across the two side keys. It was expected that, similar to the results reported by McDevitt and Williams (2003), response rate on the side key would be lower in the component with a higher reinforcer and higher response rate on the center key, and higher in the component with a lower reinforcer and lower response rate on the center key. 


\section{Experiment 2}

\section{History Building}

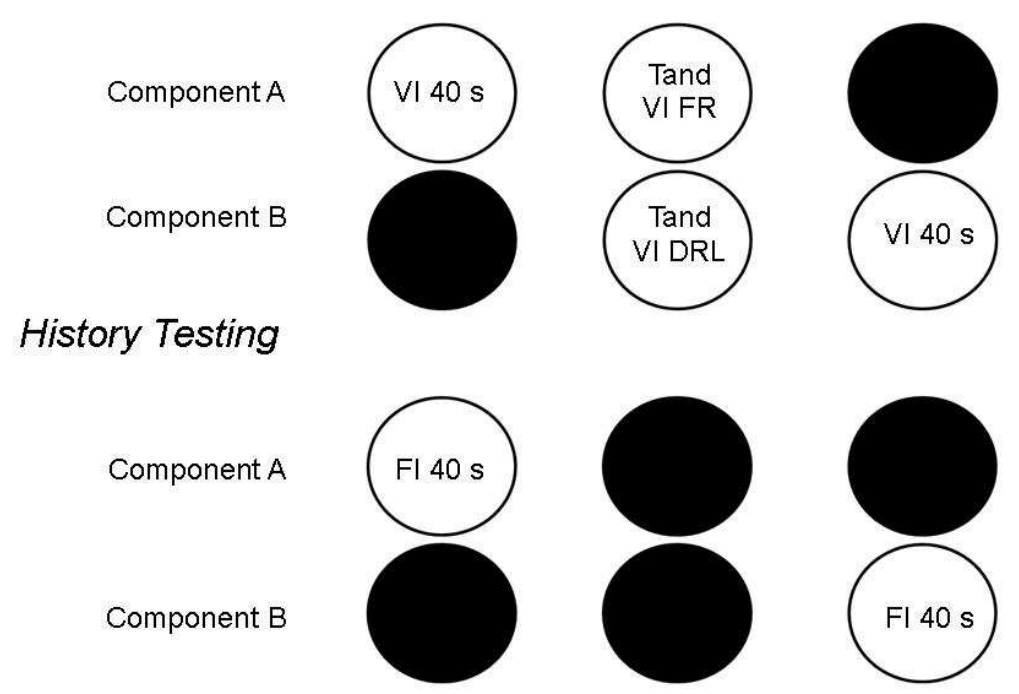

Figure 7: Illustration of history-building and history-testing conditions in Experiment 2.

The upper portion of Figure 10 illustrates the history-building condition at the terminal schedule values. Stimulusposition-schedule combinations for each pigeon are detailed in Table 8. The arrangement for Pigeon 152 provides an example: In Component A, a VI-40-s schedule was effected on the left-red side key, and a schedule designed to generate high response rates (tandem VI 20 s FR 10) was effected on the white center key (top row of Figure 10). In the tandem-VI-20-s-FR-10 schedule, after the VI interval elapsed, reinforcement was delivered after the onset of the tenth response. In Component B, a VI-40-s schedule was effected on the right-green side key, and a schedule designed to generate low response rates (tandem VI $20 \mathrm{~s}$ DRL $10 \mathrm{~s}$ ) was effected on the amber center key (second row of Figure 10). In the tandem-VI-20-s-DRL-10-s schedule, after the VI interval elapsed, reinforcement was delivered at the onset of the second of two pecks separated by $10 \mathrm{~s}$. As in Experiment 1, in each component the contingencies arranged on the center key constituted a contextual schedule designed to affect the rate of responding on the side key. There was no COD in Experiment 2.

In the first sessions of history building, VI, DRL, and FR values were adjusted on the center key in an attempt to maximize the difference in response rates between the side keys across the two components. Appendix $\mathrm{C}$ shows the number of sessions conducted with each pigeon at each value. Regardless of adjustment to the schedule values, the scheduled reinforcement rate for each of the two side keys was always VI $40 \mathrm{~s}$. As in Experiment 1, the historybuilding condition did not end for a pigeon until there were at least 20 consecutive sessions at the terminal values (and a minimum total of 57 sessions had occurred). 
Table 8: Stimulus-Position-Component Combinations for Each Pigeon in Experiment 2

\begin{tabular}{cccccc}
\hline & \multicolumn{2}{c}{ Component A } & & \multicolumn{2}{c}{ Component B } \\
\cline { 2 - 3 } \cline { 5 - 6 } Pigeon & Side & Center & & Side & Center \\
\hline 152 & Red (Left) & White & & Green (Right) & Amber \\
236 & Green (Left) & Amber & & Red (Right) & White \\
965 & Green (Left) & Amber & & Red (Right) & White \\
191 & Red (Left) & White & Green (Right) & Amber \\
\hline
\end{tabular}

History Testing. The history-testing condition was identical to that in Experiment 1, and is illustrated in the lower portion of Figure 10. The history-testing condition was conducted until: (a) a minimum of 70 sessions were completed, and (b) for the last 3 (minimum) there was a consistent difference in response rate across components (e.g. response rate on the left component was higher for three consecutive days), or (c) for a maximum of 80 sessions.

\section{Results}

\section{History Building}

Response Rate. Table 9 shows the mean and range response rates across the last 20 sessions of history building for each of the four alternatives. As expected, within each component response rates were higher on the key with the higher reinforcer rate. That is, in Component A, Pac (tandem VI FR) was greater than Pas (VI 40 s), and in Component B, Pbs (VI 40 s) was greater than Pbc (tandem VI DRL). The upper panel of Figure 11 shows the proportional response- and reinforcement-rate data for all pigeons within components. Proportional response rate within a component is the response rate on the richer alternative divided by the overall response rate (i.e., rate on the side key + rate on the center key) for that component.

Across components, response rate was greater for each pigeon on the side key in Component B than on the side key in Component $\mathrm{A}$ (Pbs>Pas). For two pigeons, 152 and 236, the magnitude of the response rate difference between the two side keys was small, but for Pigeons 965 and 191 the proportional response rates of Pbs were slightly larger: 0.59 and 0.66, respectively. These differences are summarized in Table 9 as proportional response rates (Side Keys), and are shown in the lower panel of Figure 11

Finally, overall response rate data are summarized in Table 9 as proportional response rates (Component Sums). Each pigeon had lower overall response rates in Component B than in Component A. These differences were large in all four pigeons. 
Table 9: Mean (and Range) Response Rate per Min over the Last Twenty Sessions of the History-Building Condition in Experiment 2

\begin{tabular}{|c|c|c|c|c|c|c|}
\hline \multirow[b]{2}{*}{ Subject } & \multicolumn{2}{|c|}{ Component A } & \multicolumn{2}{|c|}{ Component B } & \multicolumn{2}{|c|}{$\begin{array}{c}\text { Proportional } \\
\text { Response Rate }\end{array}$} \\
\hline & $\begin{array}{l}\text { Side Key } \\
\text { (VI40) }\end{array}$ & $\begin{array}{c}\text { Center Key } \\
\text { (TandVIFR) }\end{array}$ & $\begin{array}{l}\text { Side Key } \\
\text { (VI40) }\end{array}$ & $\begin{array}{c}\text { Center Key } \\
\text { (TandVIDRL) }\end{array}$ & $\begin{array}{l}\text { Side }^{a} \\
\text { Keys }\end{array}$ & $\begin{array}{l}\text { Comp. } \\
\text { Sums }\end{array}$ \\
\hline 152 & $13(9-15)$ & $27(17-38)$ & $13(10-18)$ & $6(2-8)$ & 0.51 & 0.33 \\
\hline 236 & $31(20-39)$ & $82(64-97)$ & $36(28-41)$ & $11(4-18)$ & 0.54 & 0.29 \\
\hline 965 & $19(14-26)$ & $80(62-101)$ & $28(20-36)$ & $12(10-16)$ & 0.59 & 0.29 \\
\hline 191 & $15(10-24)$ & $76(51-123)$ & $29(23-37)$ & $10(6-15)$ & 0.66 & 0.30 \\
\hline
\end{tabular}

${ }^{a}$ Proportional Response Rate $\left(\right.$ Side Keys) $=$ Side B $/\left(\right.$ Side B + Side A) ${ }^{b}$ Proportional Response Rate (Component Sums $)=($ Side B + Center B $) /($ Side A + Center A + Side B + Center B $)$. 


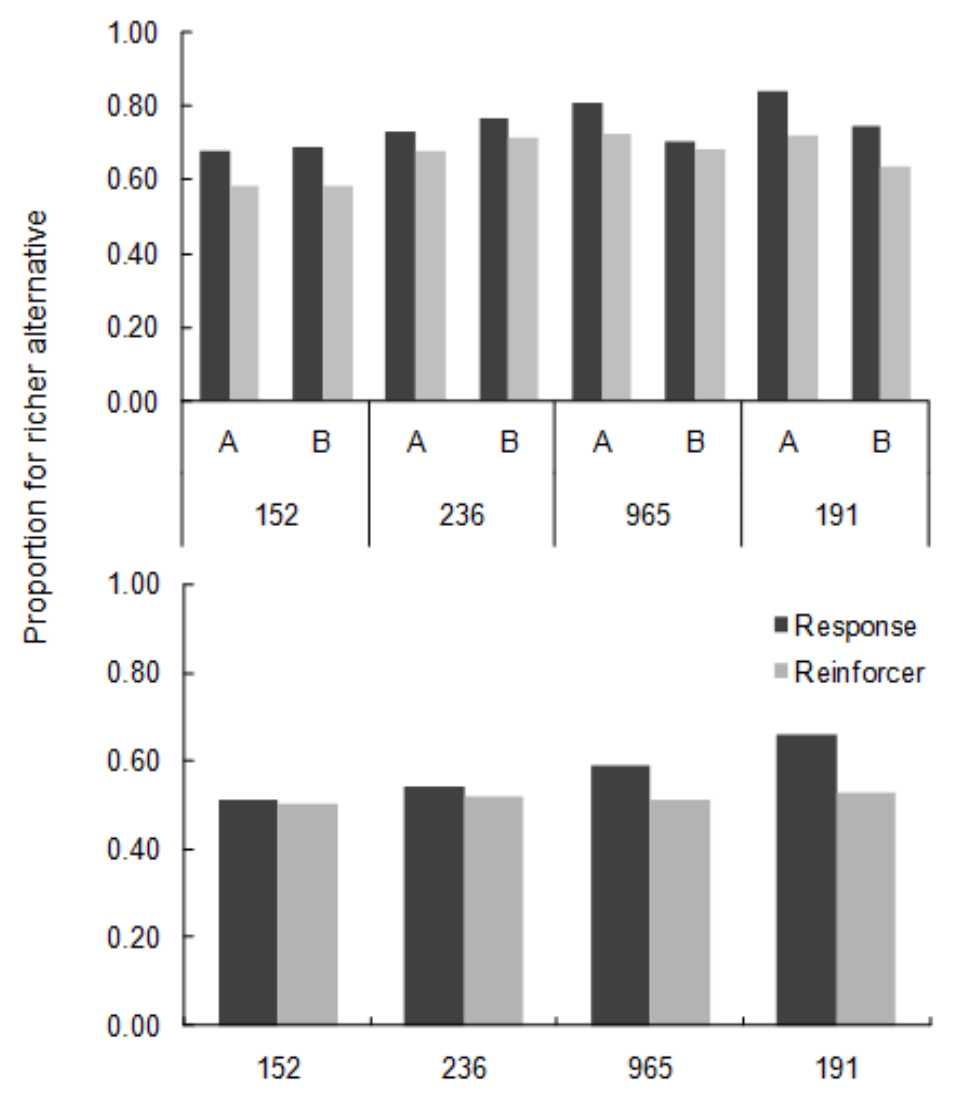

Pigeon

Figure 8: Proportional response rates and reinforcement rates in the history-building condition of Experiment 2. The upper panel shows the quotient of the response rate (dark grey) and reinforcement rate (light grey) on the richer alternative divided by the overall response rate within each component. The lower panel shows the quotient of the response rate (dark grey) and reinforcement rate (light grey) on the side key in Component B divided by the sum the response rates on the side keys in both components.

Reinforcer Rate. Mean reinforcer rates for each alternative across the two components are displayed in Table 10, and proportional within-component reinforcer rate data are displayed in the top panel of Figure 11. Reinforcer rate differences within components were similar to but generally smaller than response-rate differences. Response-rate differences within components, therefore, are greater than what would be predicted by the matching law (Herrnstein, 1970). Proportional reinforcer rate data for the side keys across components are displayed in Table 10 and illustrated in the bottom panel of Figure 11. Across components, the difference between Rbs and Ras was small (between 0.45 and 0.55). In three of the pigeons Rbs was greater than Ras. In two pigeons, 965 and 191, response-rate differences exceeded reinforcer rate differences for the two VI 40-s keys, and in the other two pigeons, response-rate differences were close to equal to reinforcer rate differences. Finally, each pigeon had a lower overall rate of reinforcement in Component B than in Component A. The proportion of overall reinforcement obtained in Component B ranged across pigeons from 0.29 to 0.41 . In three of the four pigeons, overall response-rate differences were larger than would be predicted by the matching law. These data are summarized in rightmost columns of Tables 9 and 10 . 
Table 10: Mean (and Range) Reinforcement Rate per Hour over the Last Twenty Sessions of the History-Building Condition in Experiment 2

\begin{tabular}{|c|c|c|c|c|c|c|}
\hline \multirow[b]{2}{*}{ Subject } & \multicolumn{2}{|c|}{ Component A } & \multicolumn{2}{|c|}{ Component B } & \multicolumn{2}{|c|}{ Proportional SR Rate } \\
\hline & $\begin{array}{l}\text { Side Key } \\
\text { (VI40) }\end{array}$ & $\begin{array}{l}\text { Center Key } \\
\text { (TandVIFR) }\end{array}$ & $\begin{array}{l}\text { Side Key } \\
\text { (VI40) }\end{array}$ & $\begin{array}{c}\text { Center Key } \\
\text { (TandVIDRL) }\end{array}$ & Side Keys $^{a}$ & $\begin{array}{l}\text { Comp. }^{b} \\
\text { Sums }\end{array}$ \\
\hline 152 & $78(65-85)$ & $108(85-145)$ & $76(65-90)$ & $55(20-75)$ & 0.50 & 0.41 \\
\hline 236 & $80(65-95)$ & $166(130-195)$ & 85 (70-95) & $34(10-55)$ & 0.52 & 0.33 \\
\hline 965 & $79(60-90)$ & $208(175-230)$ & $81(65-95)$ & $38(10-55)$ & 0.51 & 0.29 \\
\hline 191 & $78(66-96)$ & $197(150-252)$ & $87(72-102)$ & $50(30-72)$ & 0.53 & 0.33 \\
\hline
\end{tabular}

${ }^{a}$ Proportional Reinforcement Rate (Side Keys) $=$ Side B $/\left(\right.$ Side B + Side A) ${ }^{b}$ Proportional Reinforcement Rate $($ Component Sums $)=($ Side B + Center B $) /($ Side A + Center A + Side B + Center B $)$.

History Testing

Response Rate. Response-rate data for each pigeon in Experiment 2 are displayed in Figure 12. Each data point represents the mean response rate over a block of 10 sessions, except in pigeons for which the total number of sessions was not a multiple of 10. In these cases, the last data point is the mean of the last 10 or 11 sessions (see figure caption for details). On the left side of Figure 12, the left portion of each graph displays response rates on the side keys (Pas and Pbs) in the last twenty sessions of history building, and the right portion displays the response rates in history testing (Pas and $\mathrm{Pbs}$ ). On the right side of Figure 12, the left portion of each graph displays overall response rates in each component ( $\mathrm{Pas}+\mathrm{Pac}$ and $\mathrm{Pbs}+\mathrm{Pbc}$ ), and the right portion displays response rates in history testing (for each pigeon, the display of the history-testing data is identical on each side of the figure). In general, compared to response rate on the side keys in the history-building condition, response rates increased initially, and then fell at different rates over the course of the testing condition.

Figure 13 shows the log ratio of response rates in the first session of history testing as a proportion of the mean response rate in the same component from the last 20 sessions of history building. For each pigeon, response rate increased in each component relative to response rate in history building, and this increase was greater in Component A than Component B. This increase over history-building levels in Component A persisted for three pigeons for the first 60 sessions or more sessions of the history-testing condition. 

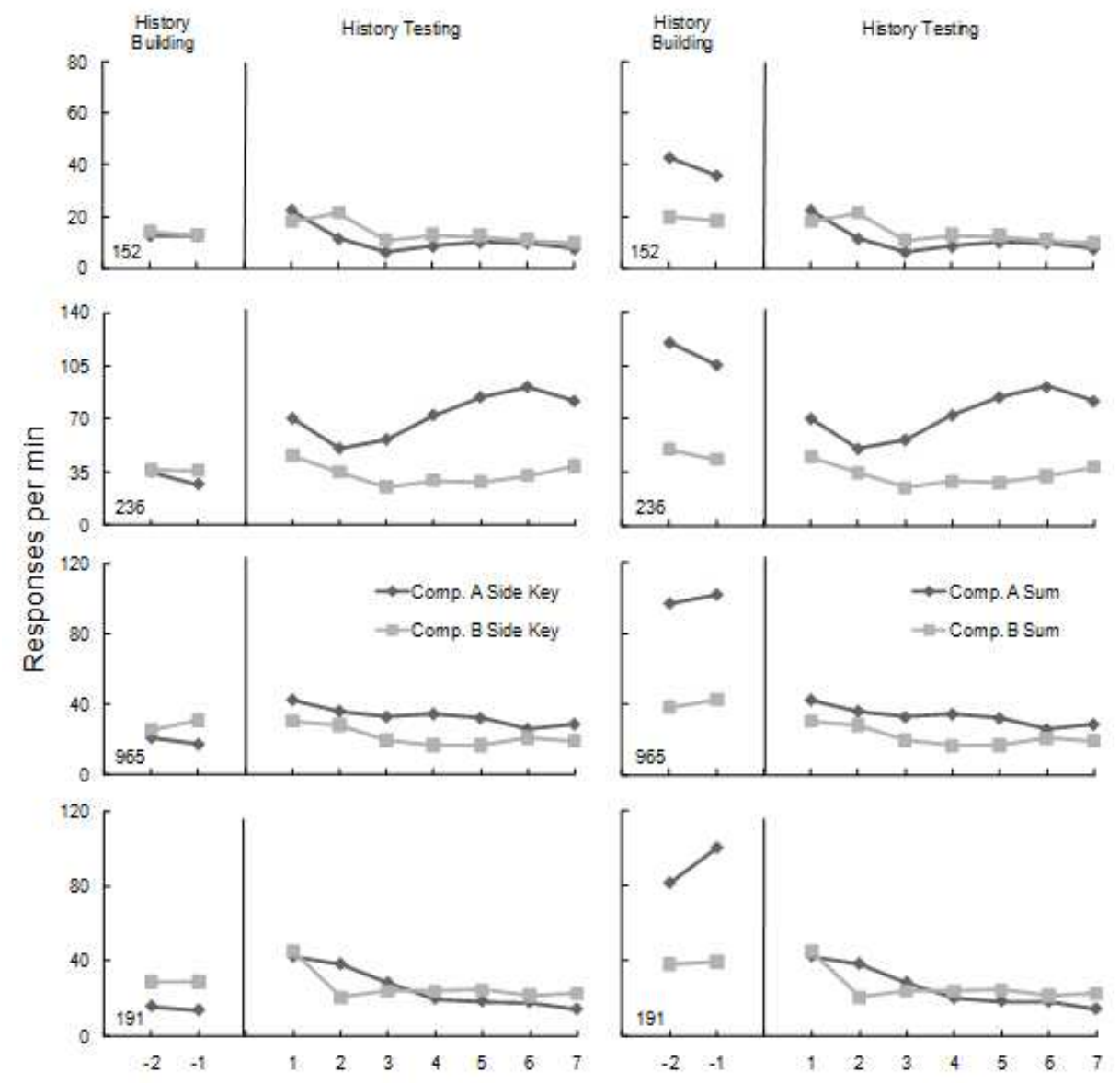

Blocks of 10 Sessions ${ }^{*}$

Figure 9: Response rate data for each pigeon in Experiment 2. In the left panel, the left portion of each graph displays response rates on the side keys (Pas and Pbs) in the last twenty sessions of history building, and the right portion displays the response rates in history testing (Pas and $\mathrm{Pbs}$ ). In the right panel, the left portion of each graph displays overall response rates in each component $(\mathrm{Pas}+\mathrm{Pac}$ and $\mathrm{Pbs}+\mathrm{Pbc})$, and the right portion displays response rates in history testing (for each pigeon, the display of the history-testing data is identical for each side of the figure).

*Each data point represents the mean response rate over a block of 10 sessions, with the following exceptions: for both 236 and 965, the last data point shows the mean of the last 11 sessions. These exceptions apply to all figures for Experiment 2 that display data in 10-session blocks. 


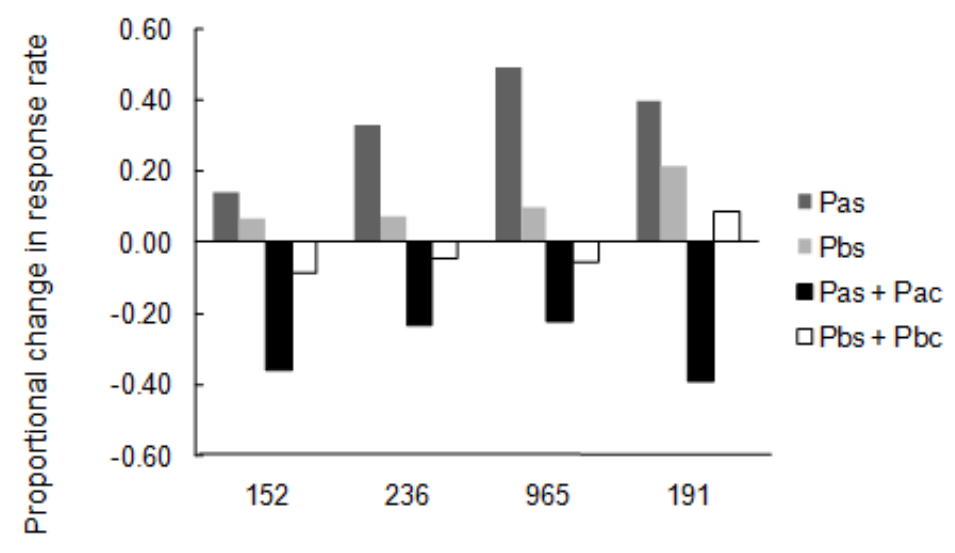

Pigeon

Figure 10: Experiment 2: Log ratio of response rates in the first session of history testing as a proportion of the mean response rate in the same component from the last 20 sessions of history building.

Response-Rate Differences. The difference in response rates in Components A and B is shown in Figure 14. On the left portion of each panel are the response rates on the side key in Component B as a proportion of side key responding in both components, and on the right portion of each panel is the proportion of responding in Component $\mathrm{B}$ in history testing. When this measure is greater than 0.50 , the response rate was greater in Component $\mathrm{B}$ than in Component A. Differences in response rates across components were not consistent across pigeons. For Pigeons 236 and 965, response rate tended to be greater in Component A, and in Pigeons 152 and 191 response rate was initially greater in Component A, and then was greater in Component B. For Pigeons 236 and 965, response-rate differences in history testing were more similar to differences in overall response rates (open diamonds) in history building than to the differences in side-key response rates. 


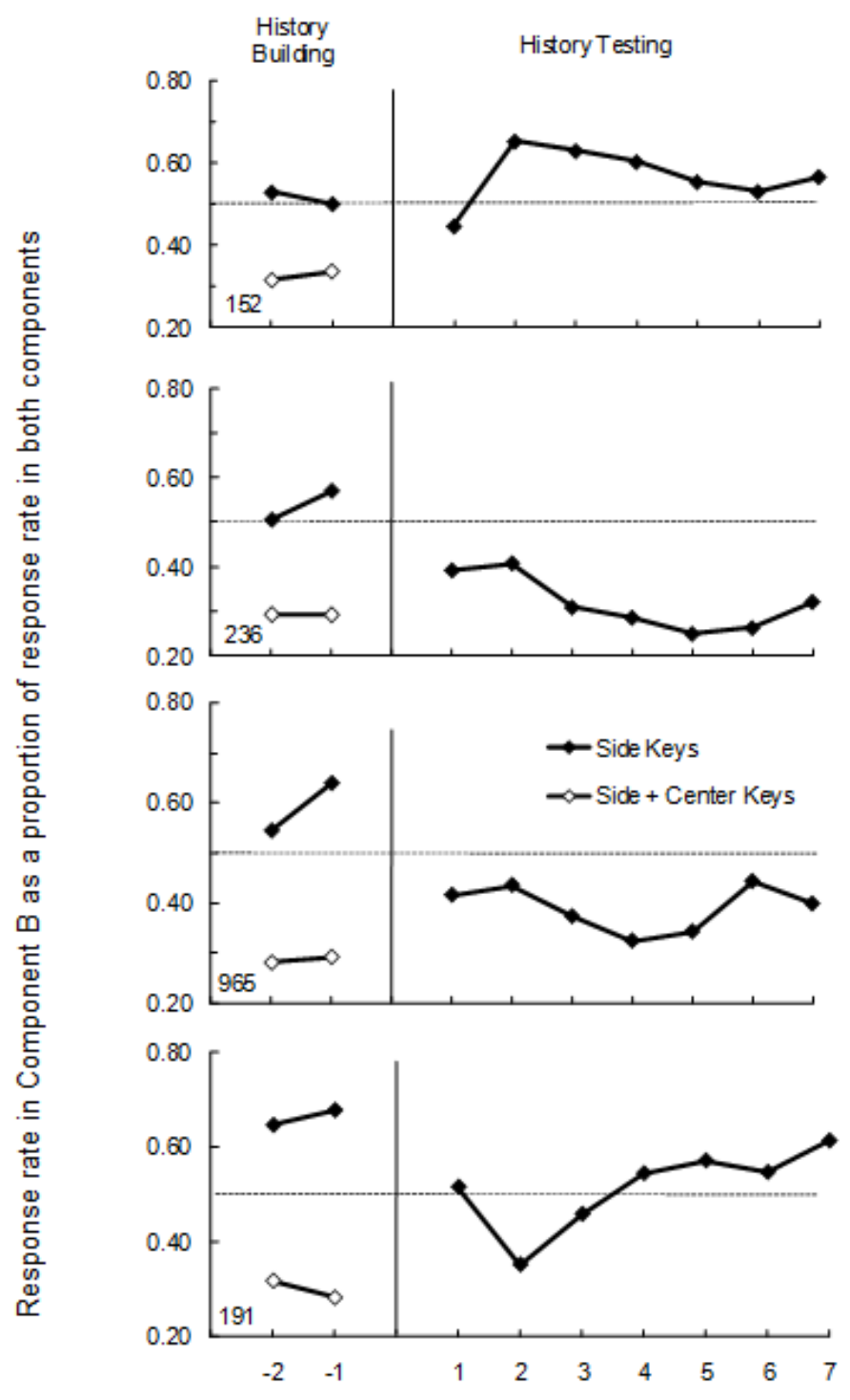

Blocks of 10 Sessions*

Figure 11: Experiment 2: difference in response rates in Components A and B. In the left portion of each panel are the response rates on the side key in Component B as a proportion of side key responding in both components $[\mathrm{Pbs} /(\mathrm{Pbs}+\mathrm{Pas})$; closed diamonds] and the response rates for overall responding in Component $\mathrm{B}$ as a proportion of all responding in both components $[(\mathrm{Pbs}+$ $\mathrm{Pbc}) /(\mathrm{Pbs}+\mathrm{Pbc}+\mathrm{Pas}+\mathrm{Pac})$; open diamonds]. On the right portion of each panel is the proportion of responding in Component $\mathrm{B}$ in history testing $[\mathrm{Pbs} /(\mathrm{Pbs}+\mathrm{Pas})]$.

Rate of Change. As in Experiment 1, rate of change was measured and compared across the two components. The rationale for and method of calculating this measure was described in the results section of Experiment 1 . The proportion of response rates in history testing to side-key response rates in history building is shown on the left side of the Figure 15, and the proportion of response rates in testing to overall response rates is displayed on the right side of the same figure. Generally, response rates in history testing were more similar to response rates on the side keys in history building than they were to the overall component rates from history building. Pigeon 236 was the exception to this finding, and in this pigeon, proportional response rates in both components were similar to overall response rates in history building. 

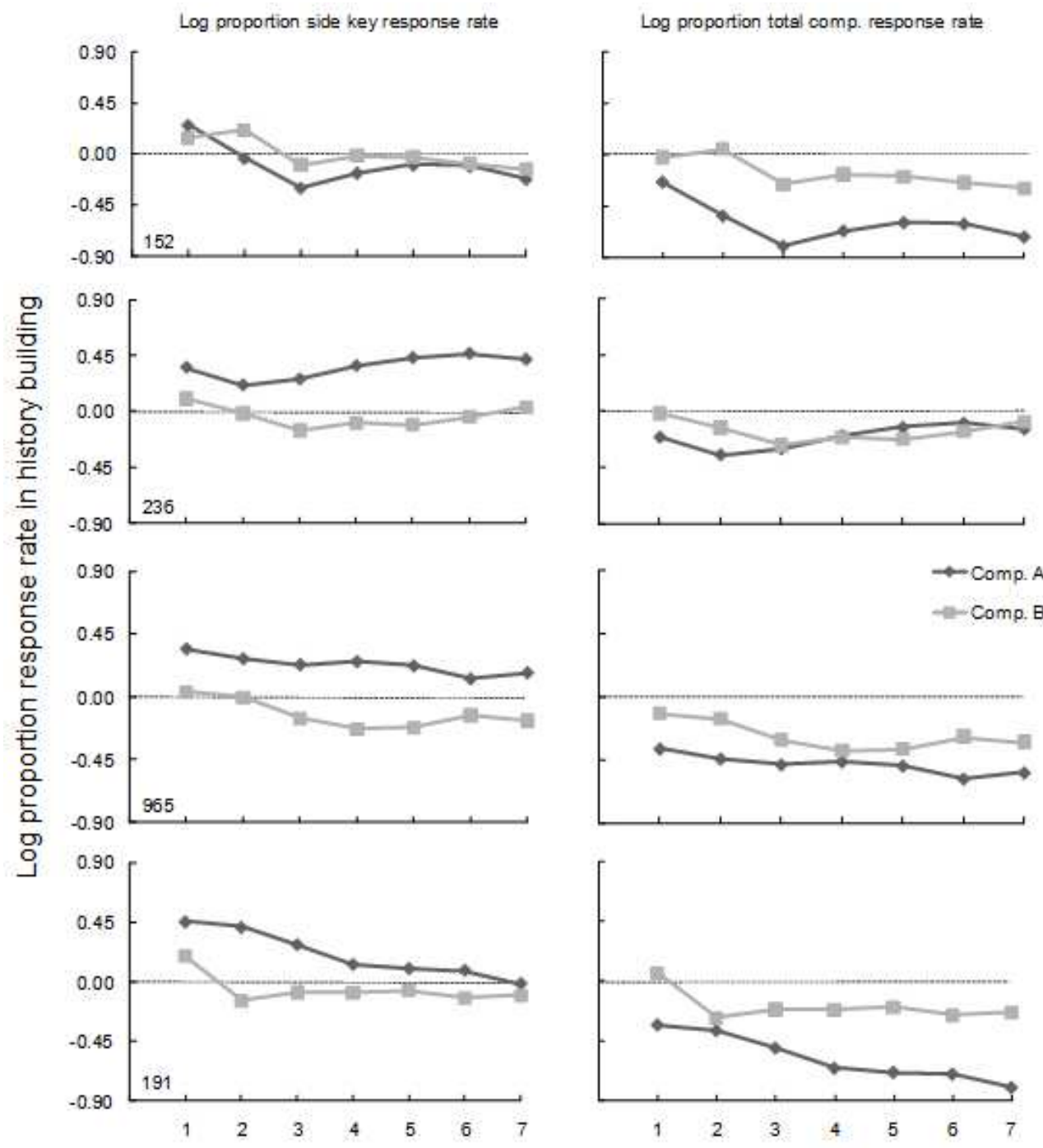

\section{Blocks of 10 Sessions*}

Figure 12: Experiment 2: log proportion of the response rate in each component of history testing. The proportion of response rates in history testing to side-key response rates in history building is displayed on the left side of the figure, and the proportion of response rates in testing to overall response rates is displayed on the right side of the figure. Side-key proportions are calculated for each component by dividing response rate in history testing by the mean response rate on the side key in the final 20 sessions of history building, and then taking the $\log$ of this ratio $\left[\log \left(\mathrm{Pas}_{\text {test }} /\right.\right.$ $\left.\mathrm{Pas}_{\text {build }}\right)$ for Component $\mathrm{A}$, and $\log \left(\mathrm{Pbs}_{\text {test }} / \mathrm{Pbs}_{\text {build }}\right)$ for Component $\left.\mathrm{B}\right]$. Overall-response-rate proportions are calculated for each component by dividing response rate in testing by mean of the overall response rate in the final 20 sessions of history building $\left[\log \left(\mathrm{Pas}_{\text {test }} /\left(\mathrm{Pas}_{\text {build }}+\mathrm{Pac}_{\text {build }}\right)\right.\right.$ for Component $\mathrm{A}$, and $\log \left(\mathrm{Pbs}_{\text {test }} /\left(\mathrm{Pbs}_{\text {build }}+\mathrm{Pbc}_{\text {build }}\right)\right.$ for Component $\left.\mathrm{B}\right]$. Log-proportion data are presented in 10-session blocks.

Figures 16 and 17 allow a comparison of the rate of change in response rate in the two components. Most of the change in response rate in history testing occurred in the first 20 sessions of that condition. Figure 16 shows the slope of the log ratios of response rates on the side keys for each pigeon in the first 20 sessions of testing (left panel) and for the all of the test sessions (right panel). Slopes generally were steeper over the first 20 sessions than over the 
entire course of the testing condition, with the exceptions of Component B in Pigeon 152, and Component A in Pigeon 191. Session-by-session response-rate data for the first 20 sessions are displayed in Figure 17.

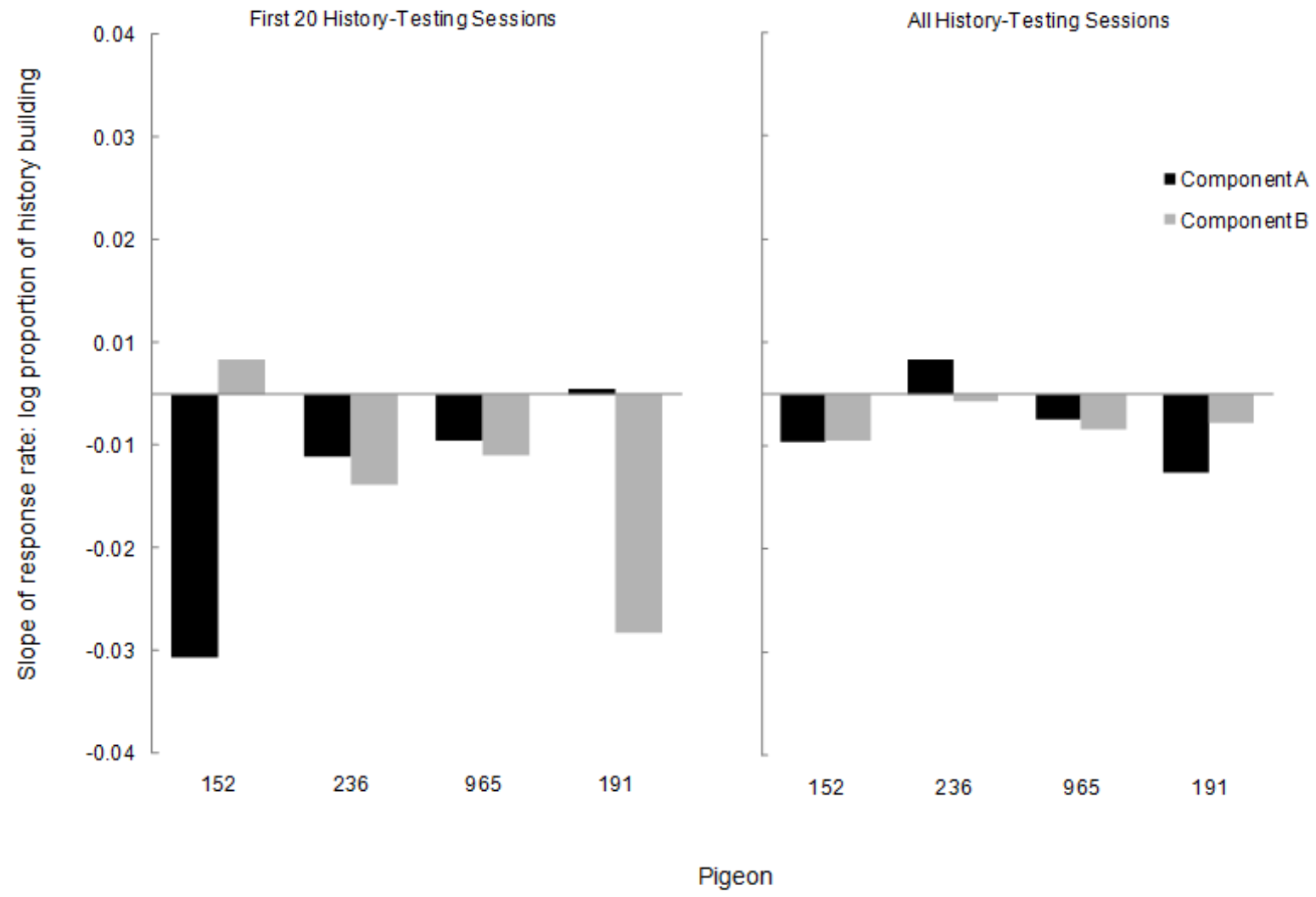

Figure 13: Figure 16. Experiment 2: slope of the log ratios of response rates on the side keys for each pigeon in the first 20 sessions of testing (left panel) and for the all of the test sessions (right panel). To calculate these slopes, the mean of the last 20 sessions of history building was included as the first data point, and individual session data were used for the history-testing condition. 


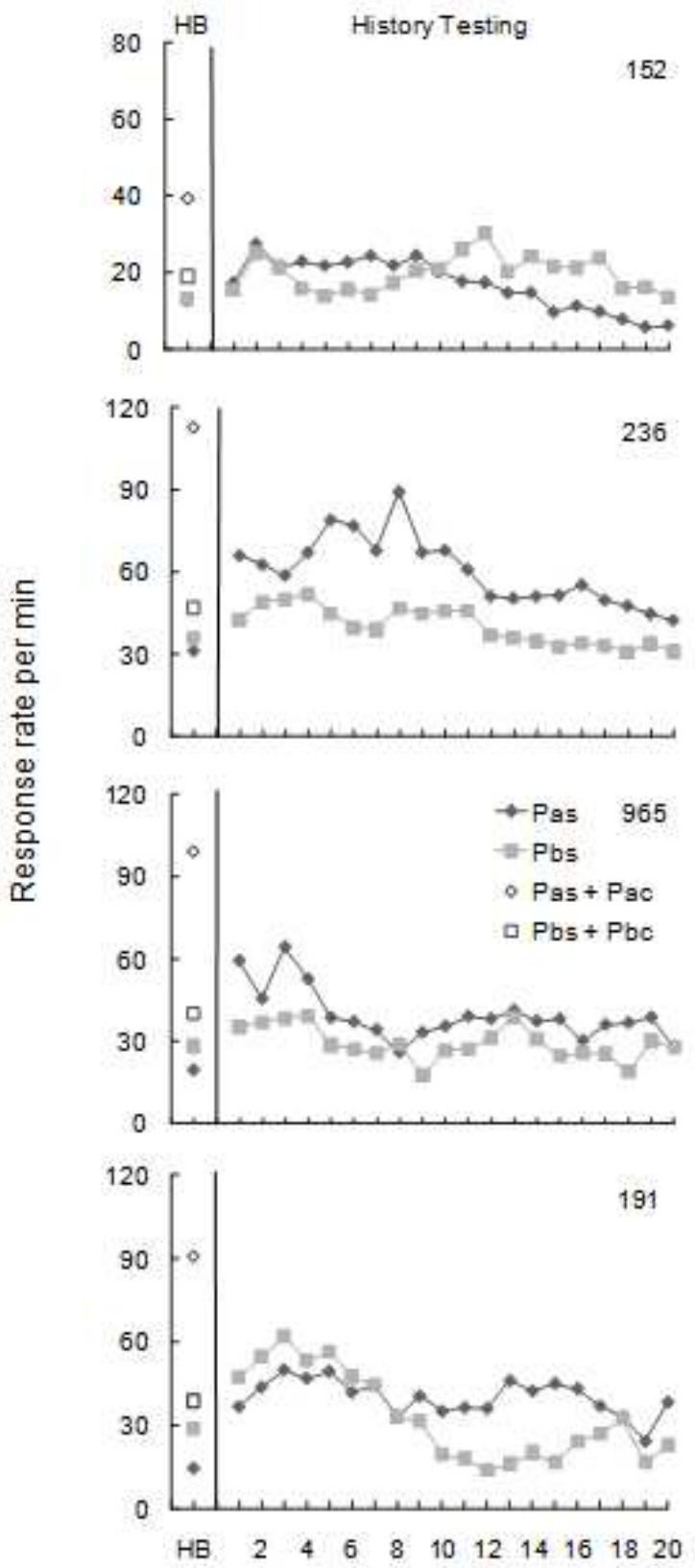

\section{Sessions}

Figure 14: Experiment 2: session-by-session response-rate data for the first 20 sessions.

Pattern of Responding. As can be seen in Figure 18, for each pigeon, positively accelerated responding developed in both components, and in three pigeons the mean QL for the first 10 sessions was greater in Component B than A (though only slightly so in Pigeon 191). In both components the QL continued to increase after these first sessions. In three of the four pigeons the maximum 10-session mean QL in Component B was greater than or equal to that in Component A. 


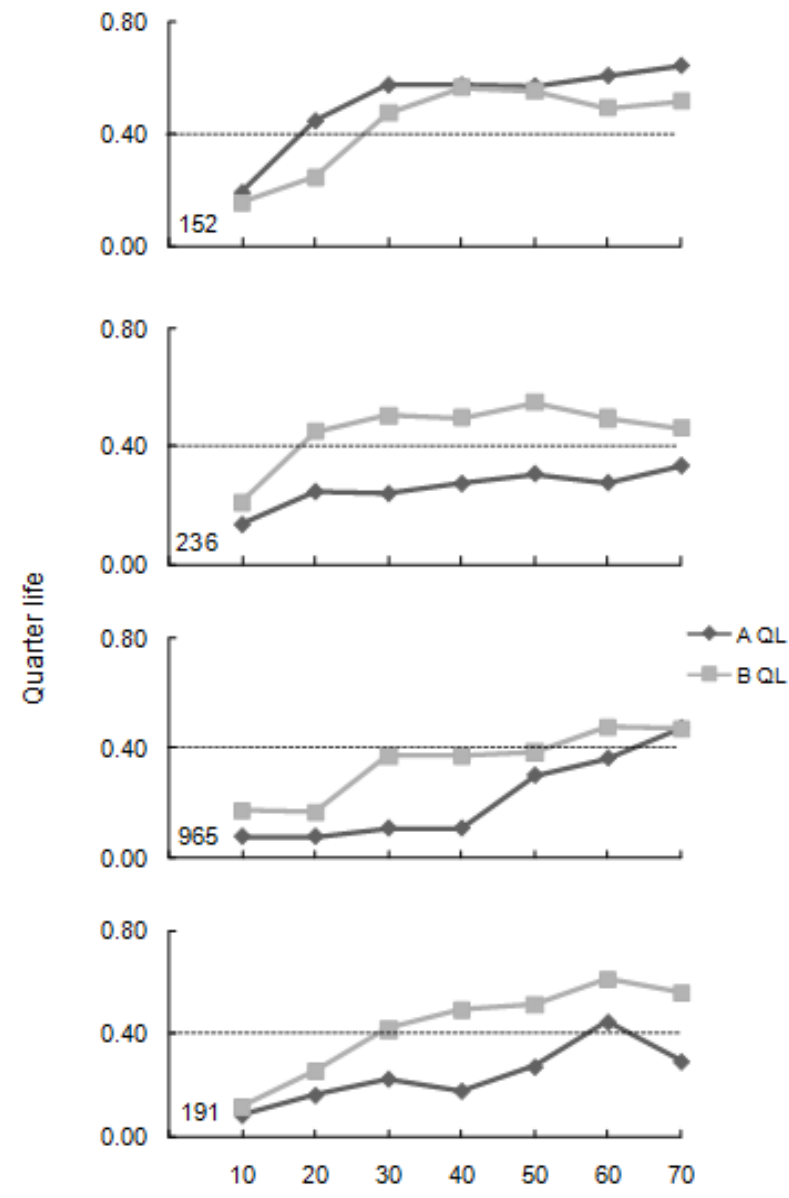

Blocks of 10 Sessions*

Figure 15: Experiment 2: mean QL in each component of the history-testing condition in 10session blocks.

Other Measures of Persistence. Table 11 displays several measures of persistence that allow comparison of features of responding between the components in the history-testing condition. These measures were described in the Results section of Experiment 1. In the last sessions of history building, each pigeon responded at a higher rate on the side key in Component B than A. In history testing each pigeon had a higher rate of keypecking in Component A than B in at least one of the first 10 sessions. Another measure for which results were consistent across pigeons was the $10 \%$ terminal measure of convergence. Pigeon 965 was the only pigeon to reach this criterion within 68 sessions. Finally, in three of the four pigeons, it took fewer sessions for QLs to increase to at least 0.40 (for at least five consecutive days) in Component B than A. There is inconsistency between pigeons on the number of sessions for which the response rate in Component $\mathrm{B}$ is greater than that in Component $\mathrm{A}(\mathrm{B}>\mathrm{A} / \mathrm{total}$ test sessions), number of sessions to convergence of response rate between the two components (10 Resp / min and 10\% Range), and measures of standard deviation of response rates across sessions. 
Table 11: Measures of Persistence for Experiment 2

\begin{tabular}{|c|c|c|c|c|}
\hline \multirow[b]{2}{*}{ Measure of Persistence } & \multicolumn{4}{|c|}{ Subject } \\
\hline & 152 & 236 & 965 & 191 \\
\hline $\mathrm{B}>\mathrm{A} /$ total test sessions & $54 / 70$ & $0 / 71$ & $2 / 70$ & $46 / 70$ \\
\hline Sign change 1 & 10 & 1 & 1 & 7 \\
\hline Sign change 2 & 41 & $>71$ & 9 & 28 \\
\hline 10 Resp /min & 5 & $>71$ & 56 & 5 \\
\hline $10 \%$ Range & 54 & $>71$ & 56 & 8 \\
\hline $10 \%$ terminal: A & $>70$ & $>71$ & 35 & $>70$ \\
\hline $10 \%$ terminal: $\mathrm{B}$ & $>70$ & 68 & 61 & $>70$ \\
\hline 1st Stable (no. sessions) & $8(2)$ & $9(1)$ & $20(2)$ & $6(1)$ \\
\hline 2nd Stable (no. sessions) & $27(1)$ & $17(2)$ & $42(1)$ & $23(1)$ \\
\hline SD: A (1st 10 sessions) & 2.75 & 8.98 & 12.49 & 5.82 \\
\hline SD: B (1st 10 sessions) & 3.72 & 4.27 & 6.88 & 13.20 \\
\hline $\begin{array}{l}\text { Slope of change in SD: A } \\
\text { (10 session blocks) }\end{array}$ & -0.35 & -0.07 & -1.19 & -0.62 \\
\hline $\begin{array}{l}\text { Slope of change in SD: B } \\
\text { (10 session blocks) }\end{array}$ & -0.30 & -0.37 & -0.72 & -1.15 \\
\hline $\begin{array}{l}\mathrm{QL}>=0.40: \mathrm{A} \\
\text { (1st instance) }\end{array}$ & 13 & 44 & 47 & 30 \\
\hline $\begin{array}{l}\mathrm{QL}>=0.40: \mathrm{B} \\
\text { (1st instance) }\end{array}$ & 19 & 10 & 24 & 19 \\
\hline $\begin{array}{l}\mathrm{QL}>=0.40: \mathrm{A} \\
(5 \text { consec days })\end{array}$ & 20 & $>71$ & 62 & 57 \\
\hline $\begin{array}{l}\mathrm{QL}>=0.40: \mathrm{B} \\
(5 \text { conseq days })\end{array}$ & 25 & 14 & 32 & 34 \\
\hline
\end{tabular}




\section{Discussion}

In Experiment 2 reinforcement for alternative responses was delivered in attempt to generate a behavioral history of high- and low-rate responding, using equal schedules of reinforcement. Effects of this history on subsequent responding under a novel contingency were examined in the history-testing condition. Contextual schedules of reinforcement operating on alternative responses were designed to affect response rate on the target, common schedule responses in two components of a multiple schedule. As described earlier, previous studies of behavioral history that have sought to generate response-rate differences with equal rates of reinforcement have typically used rate-controlling schedules (such as DRL, DRH, and FR) in their history-building components. These contingencies may have confounded the effects of historical low- vs. high-rate responding with the schedules used to generate these rates. In the current experiment, rate-controlling schedules were used on alternative response keys, providing a context designed to generate response-rate differences on the side keys, the target responses for history testing. The contextual schedules in Experiment 2 were tandem VI FR in Component A, designed to decrease response rate on the target response (Pas), and tandem VI DRL in a Component B, designed to increase response rate on the target response (Pbs). Large, consistent response-rate differences were not achieved in history building.

Effects of behavioral history were evident in several features of responding in the history-testing condition: 1) between-component response-rate differences of greater than $10 \%$ that persisted for $8,54,56$, and $>70$ sessions in Pigeons 191, 152, 965, and 236, respectively; 2) an initially greater rate of change in response rate in Component A than Component B in three of four pigeons; 3) between-component differences in rate of change of response rate over the course of history testing in three of the four pigeons; 4) failure of response rates in both components to be within $10 \%$ of terminal response rates in the respective component within 67 sessions in three of the four pigeons; and 5) a greater rate of increase in QL in Component B than A over the first 10 sessions and larger QLs in Component $\mathrm{B}$ throughout history testing in three of four pigeons.

Although the history effects that occurred in this study varied across pigeons, the historical differences between the contexts of the two components did have some lasting effects on responding. The primary purpose of this experiment, however, was to investigate effects of historically different rates of responding generated by equal schedules of reinforcement. As shown in Appendix C, in attempt to achieve response-rate differences similar to those reported by McDevitt and Williams (2003), adjustments were made to the schedules of reinforcement in the history-building condition. Similar to Experiment 1, however, response-rate differences on the side keys (Pas vs. $\mathrm{Pbs}$ ) in history building were not consistently large. Because response-rate differences between the commonschedule side keys in history building were not consistent in all pigeons there was again no clear way to evaluate the relative effect of response vs. reinforcement variables on subsequent responding. As in Experiment 1, the question was whether the historical contextual differences between the two components would have different and persistent effects on responding when the contingencies in both components were changed to the same schedule of FI reinforcement.

In the first testing session of the current study three of the four pigeons responded at a higher rate in the presence of the Component A side-key stimulus (that had been presented previously in the context of the tandem VI-FR alternative) than in the presence of the Component B side-key stimulus. The pattern of higher response rates in Component A persisted throughout the experiment for two pigeons (236, and 965). For the other two pigeons, response rate in Component B exceeded that in Component A in most of the remaining sessions (Pigeon 152: 54/70, and Pigeon 191: 46/70 sessions).

The original question of the degree to which historical response rate vs. reinforcement rate exerts persistent control over subsequent responding is best addressed in this experiment with an analysis of the response rates of Pigeons 965 and 191, because these were the two pigeons with the greatest difference between response rates on the side keys in history building. There are few similarities between these two pigeons in history testing. In the case of Pigeon 965, response rates tended to be greater in Component A, and in the case of Pigeon 191, response rates tended to be greater in Component B. In Pigeon 191, response-rate differences were in the same direction (Pbs > Pas) in the first 6 sessions of history testing as they were in the last sessions of history building. In no case, however, is there clear persistence of response-rate differences into the history testing condition. It seems that some feature of the history-building condition affected responding for many sessions into the history-testing condition, however, as in Experiment 1, the determinants of response rate in history testing are not clear. 
Chapter 4: Comparison of Results from Experiments 1 and 2

Despite the use of almost identical procedures, the response-rate differences obtained by McDevitt and Williams (2003) were not obtained in history building in Experiment 1. The lack of differences in response rates in Experiment 1 led to the design of Experiment 2, which, in attempt to exert stronger control over response rates on the target keys, programmed rate-controlling contingencies for the alternative responses in each component. Other than these rate-controlling contingencies Experiments 1 and 2 are almost identical to one another. It is possible, therefore, that differences in results from the two experiments may be a function of the presence or absence of ratecontrolling contingencies on the alternative response key. A discussion of the similarities and differences between results in Experiments 1 and 2 is presented in this section, and this discussion is followed by a consideration of features of the history-building and history-testing conditions that may have influenced responding in both experiments.

The results of Experiments 1 and 2 were similar in several ways: (1) The history-building condition for neither resulted in large response-rate differences on the common-schedule keys; (2) in both experiments there were response rate increases in history testing, and these increases were larger in Component $A$ (with the rich alternative key) than Component B (with the lean alternative key); (3) response-rate differences persisted from 8 to $>67$ sessions, and this range was similarly large in each experiment; (4) between-component differences in the rate of change of response rates were greater early in the testing condition than they were later in the testing condition; (5) in no case did response rates within components reach the criterion for stability at their terminal level in fewer than 67 sessions; (6) there was no relation between the difference in historical response rate in Components $\mathrm{A}$ and $\mathrm{B}$ and the tendency to respond at higher rates in Component B in history testing; and (7) there was a negative relation between the increase in response rate in Component $\mathrm{A}$ in the first session of testing, and the number of sessions of testing for which the response rate was higher in Component B than A. Specifically, the closer response rate was in Component $\mathrm{A}$ in the first session of history testing to the overall rate of responding (Pas $+\mathrm{Pac}$ ) in Component $\mathrm{A}$ from history building, the fewer sessions there were in history testing with greater response rates in Component B. Data from the eight pigeons in Experiments 1 and 2 are displayed in a scatterplot in Figure 19. Plotted on the ordinate for each pigeon is the result of the following calculation: the number of history-testing sessions in which $\mathrm{Pbs}_{\text {test }}$ was greater than $\mathrm{Pas}_{\text {test }}$, divided by the total number of history-testing sessions. Plotted on the abscissa is $\mathrm{Pas}_{\text {test }} /\left(\mathrm{Pas}_{\text {build }}+\mathrm{Pac}_{\text {build }}\right)$ for the first session of history testing. The Pearson product-moment correlation of these values was statistically significant in Experiment $2[r(2)=-0.96, p<.05]$. The pigeons for which response rate in Component $\mathrm{A}$ in the first session of history testing was more similar to the overall response rate from history building were less likely to respond at higher rates in Component B, at any time over the course of history testing.

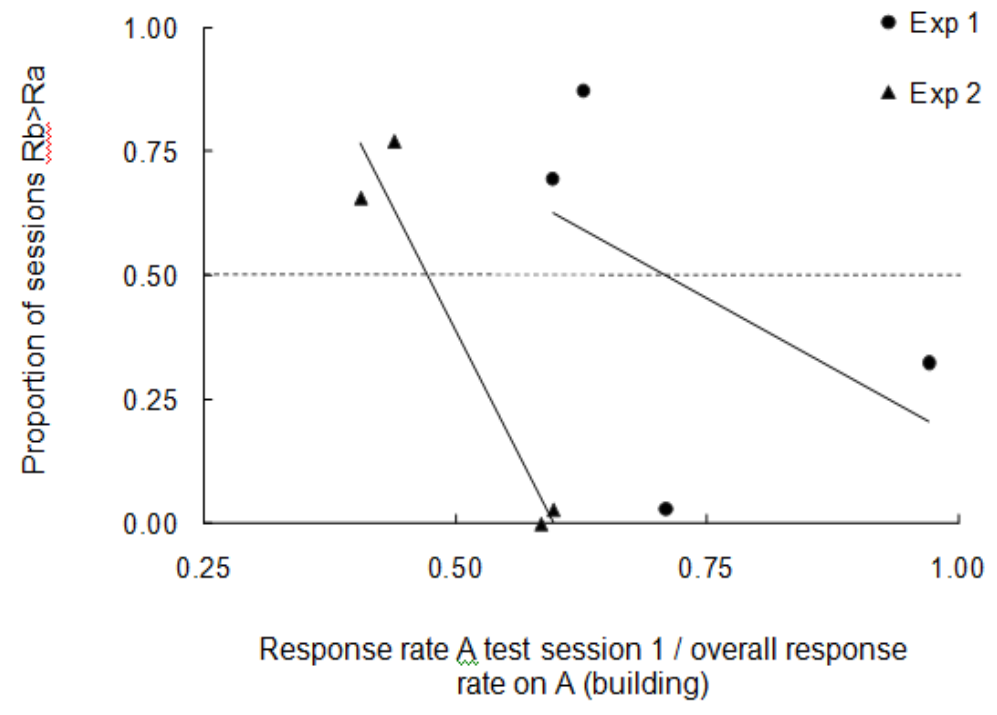

Figure 16: Scatterplot of data from Experiments 1 and 2. On the ordinate for each pigeon is number of history-testing sessions in which $\mathrm{Pbs}_{\text {test }}$ was greater than $\mathrm{Pas}_{\text {test }}$, divided by the total number of history-testing sessions. On the abscissa is $\mathrm{Pas}_{\text {test }} /\left(\mathrm{Pas}_{\text {build }}+\mathrm{Pac}_{\text {build }}\right)$ for the first session of history testing. 
Several of these results indicate inconsistent sources of control in the history-testing condition. Findings 4 and 5 , listed above, suggest that sources of control may be inconsistent over the course of contact with the history-testing condition. For example, it is possible that the differences in response rate were, early in history testing, a result of the overall rate of reinforcement in the history-building condition, and, later in history testing, a result of the interaction between historic response rates and the contingencies related to the new schedule. McLean and Blampied (1995) in their analysis of behavioral resistance to reinforcement suggested a comparison not only between rates of change in the initial sessions of testing, but also of asymptotic level relative to baseline, and temporal distance to this level.

Findings 6 and 7, listed above, show that the control mechanisms are inconsistent between pigeons. It was expected that the differences in response rates would persist to some extent in the history-testing conditions, as in previous experiments in behavioral history (e.g., Freeman \& Lattal, 1992; Metzger, 1992). In Experiments 1 and 2 of the current study, although the difference in response rates on the target keys in history building was generally not large, in each case, Pbs was greater than Pas. In history testing the direction of this difference reversed in the first session in five of eight pigeons, and within the first 10 sessions in all eight pigeons. The presence of these early reversals in response rate suggests that in history testing, something other than simply historic response or reinforcement rate on the target keys was controlling responding. It seems instead that the responding of individual pigeons was affected differently by the contextual schedules of reinforcement in history building. Pigeons 975 and 685 in Experiment 1, and 236 and 965, in Experiment 2 exhibited response rates in the first test session that more nearly approximated the overall rate of responding in Component A in history building. As discussed above and shown in Figure 19, these were the same pigeons with the fewest test sessions in which response rate in Component $\mathrm{B}$ exceeded that in Component A. That this result is related within pigeons to the extent of increase in response rate in Component $\mathrm{A}$ during the first test session suggests that in these pigeons, the concurrent schedules in each component may have functioned as a single schedule, and the overall response (or reinforcement) rate was more predictive of subsequent responding than the rates on the side keys per se. In the other pigeons, the concurrently available alternatives in each component may have functioned more independently from one another, and responding on the target key in these cases was more predictive of responding in the subsequent history-testing condition.

There were two notable differences in the results of Experiments 1 and 2: (1) in Experiment 1, undermatching was seen within the components of the history-building condition, and in Experiment 2, overmatching was seen within the same components; and (2) positively accelerated responding (as measured by QL) tended to be initially greater in Component A in Experiment 1, and was initially greater and developed more quickly in Component B in Experiment 2. Additionally, positively accelerated responding developed more gradually in Component A in Experiment 2 than in Experiment 1. The procedures used in Experiments 1 and 2 were nearly identical to one another, with the exception of the use of rate-controlling contingencies on the alternative response (center) keys in Experiment 2. Therefore, differences in the results of the two studies may be a function of these rate-controlling contingencies. The overmatching finding from Experiment 2, in contrast to Experiment 1, can be taken as evidence that the rate-controlling contingencies in Experiment 2 appear to have controlled more directly response allocation than the concurrent VI schedules in Experiment 1. Additionally, the development of QLs in Component B of Experiment 2 can be interpreted in two ways: (1) the historical DRL contingency on the alternative key in that component accelerated the development of characteristically patterned FI responding in history testing, or (2) responding in Component B was less resistant to change than in Component A. Response rate data do not support the second interpretation, and so it is possible that the rate-controlling contingencies on the alternative keys in each component of history-building in Experiment 2 are responsible for the differential time to development of typical FI patterns of responding in three of the four pigeons. Investigators who have used FR and DRL contingencies on target responses in multiple schedules have reported no differences in the development of QLs across components (e.g., Freeman \& Lattal, 1992; Metzger, 1992). One experiment that compared effects of schedule histories using different groups of subjects, however, found that FI-typical patterns developed more quickly in a group that had a history of DRL schedules, than in a group that had a history of FR schedules (Urbain et al., 1978). Studies of behavioral history that used schedules other than DRL and FR have found that post-reinforcement pausing (PRP) developed more rapidly when an FI component was preceded by an FT component (Lopez \& Menez, 1995); and that FI scallops developed more quickly if the FI condition had been preceded by a different schedule in a previous condition (VR; Wanchisen, Tatham, \& Mooney, 1989) or by more than one other schedule condition (both FR and DRL; Cole, 2001). Cole (2001) asserted that pattern of responding might be a more sensitive measure of schedule history effects than response rate - although the asymptotic patterns might look similar across conditions, historical conditions may influence the rate at which asymptote is reached. In Experiment 2 of the present study, the presence of the DRL contingency on the alternative key may have accelerated the development of response patterns in 
Component B, and the presence of the FR contingency on the alternative key may have retarded the development of response patterns in Component A. It remains unclear why this effect would occur in the current study, with multiple-concurrent schedules in history building condition, and not in previous studies with multiple schedules (e.g., Freeman \& Lattal, 1992; Metzger, 1992).

Figure 20 shows the log of the response rate in each session as a proportion of the average response rate on the respective side key over the last 10 sessions on history testing. In most of these cases, response rate was generally more similar to response rate on the side key in history building in Component B. This finding is more consistent across pigeons in Experiment 2 than 1. Results are difficult to interpret, however, because of the difference in the variability across components, and over the course of the history-testing condition. Greater resistance to change in Component B would be inconsistent with of the findings of previous studies that have shown that lower rate responding is more resistant to change (Blackman, 1968; Metzger, 1992; Lattal, 1989; Nevin, 1974, Experiment 5; Nevin et al., 2001; Tatham et al., 1993; Urbain et al., 1978), but the degree to which resistance Experiments 1 and 2 can be taken to have replicated this finding is questionable.
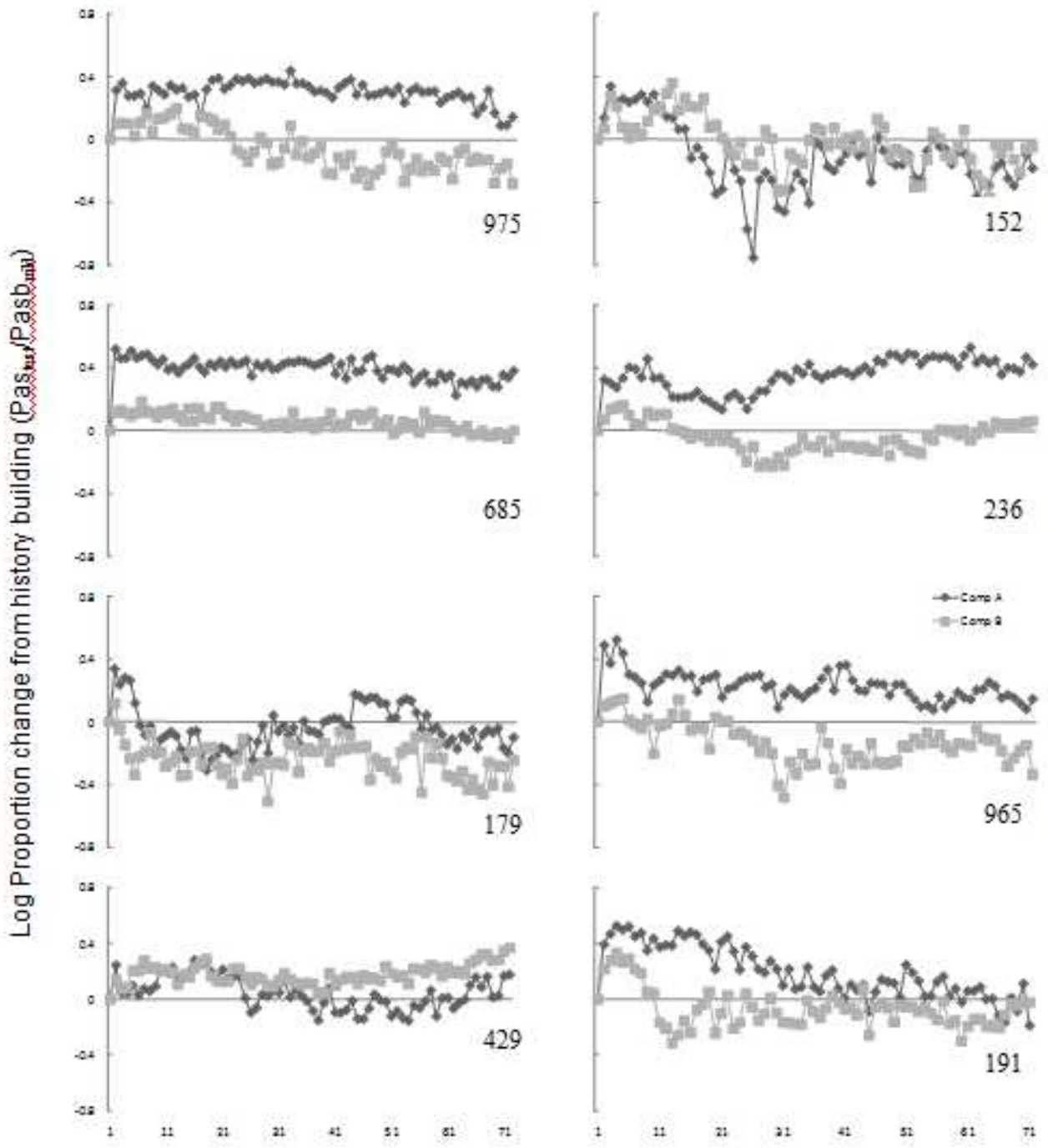

Sessions

Figure 17: Log of the response rate in each session as a proportion of the average response rate on the respective side key over the last 20 sessions on history testing in Experiment 1 and 2. 
Experiments 1 and 2 did not replicate the findings of experiments that used procedures similar to the present history building-conditions, and then tested for preference by presenting the two common-schedule keys together (e.g., Belke, 1992; Bell \& Williams, 2002; McDevitt \& Williams, 2003; McDevitt \& Bell, 2008, continuous-trial probes; Williams \& Bell, 1996, Experiment 1). In these in concurrent-schedule probes more responding occurred on the response key that had been relatively rich in the training context. To the extent that this type of "preference" can be generalized to response rates on response keys presented individually in a multiple schedule, findings from the above studies would predict that, at least in the first part of history testing, a higher rate of responding would occur on the side key in Component B than A. Response rate was greater in Component B than A in the first session of history testing for only one pigeon in Experiment 1, and one pigeon in Experiment 2.

Behavioral momentum theory (cf. Nevin and Grace, 2000; Nevin et al., 1990) would predict the response from the component with the greater amount of reinforcement to be more resistant to disruption, as a function of the Pavlovian contingency between component-specific stimuli and the total amount of reinforcement obtained in each component; in this case, responding in Component A would be more resistant to change. An important difference between the current procedure and those which have led to this prediction is that in the current case, the disruptor was a new schedule of reinforcement. This and several additional issues related to interpreting the data from Experiments 1 and 2 in light of behavioral momentum theory now will be considered.

Differences between history-building and history-testing conditions can be responsible to some extent for effects of historical factors on subsequent behavior, and therefore these differences must be considered when interpreting results. There are several features of the procedures in Experiments 1 and 2 that contribute to difficulty in interpreting results. These features include (1) changes in the number of available operants, (2) changes in stimuli, and (3) changes in the rate and periodicity of reinforcement.

First, in each component of both experiments, the number of operative, illuminated keys changed from two in history building, to one in history testing. This change affected the pattern of responding in each component. Whereas pigeons previously moved between two keys, pecking each of them at different times; in history testing the behavior of pecking a lighted center key was no longer available. Additionally, the behavior of moving back and forth between pecking two illuminated, operative keys was no longer available. Although moving about the chamber was not measured, pecking the center key in history testing occurred at near-zero rates for all pigeons. This change in conditions can be considered to be greater in Component A than B, because in Component A more reinforcement was delivered for pecking the center key, and more pecking was allocated to that key. Along these lines, effects of changing the number of available operants should be greater in Component A. In most cases, response rate increased more in Component A than B. Could changing the number of available (illuminated and operative) response keys in history testing have contributed to this disproportional increase in response rate? There is evidence that the preference probe tests used by Belke (1992) and others were influenced by the pattern of switching between response keys. In probe trials, when the common schedule keys were concurrently available there was more responding on the key that had been relatively rich in the training condition. Doubt has been raised as to whether this responding indicated the relative value of the stimuli as a function of their historical context per se, as Belke (1992) and McDevitt \& Williams (2003) have suggested, or whether the "preference" of that key was confounded with the carryover of an historical pattern of responding; a tendency to move away from the key that had been relatively lean and toward the key that had been relatively rich (Gibbon, 1995; Grace \& Savastano, 1997; McDevitt \& Bell, 2008; Nevin \& Grace, 2000; Zentall, Weaver, \& Sherburne, 1996; Williams \& Bell, 1996, Experiment 2).

Responding is inversely related to the rate of concurrently available reinforcement (Catania, 1963; Rachlin \& Baum, 1972). The greater increase in response rate in most pigeons in Component A could be expected in light of this finding. Perhaps the increased response rate seen in most pigeons in the history-testing condition in Experiments 1 and 2 of the present study resulted from a reorganization of responding within components. Response rates on the side keys in history testing could have resulted from a reallocation of responding from the center key (and also, perhaps, the switching response) to the side key. Thus, in addition to the persistence of responding that had been reinforced on the side key was some portion of other responding that had previously occurred in the same component. This interpretation is similar to the behavioral reallocation hypothesis of behavioral contrast in multiple schedules (Hinson \& Staddon, 1978; McLean, 1992), which hypothesizes all multiple schedules to be multipleconcurrent schedules, in which extraneous responding is maintained by extraneous reinforcement. These extraneous variables compete with the target response and are responsible for the behavior contrast that can occur when the reinforcement schedule in one component is changed. For example, if one component of a multiple VI VI is changed to EXT, the subject will be free to engage in "extraneous" behavior in that component, without competition from the target response. This engagement in extraneous behavior in the EXT component will reduce the value of extraneous 
reinforcement in the unchanged component. As a result, the subject will engage less in the extraneous response in the unchanged component, thereby reducing the competition for the target response, and the target response will increase. In the present set of experiments, extraneous reinforcement was programmed, and extraneous responding was measured. It makes sense that response rate would increase should the concurrently available reinforcement be eliminated, and that this increase would be greater in the component with the historically greater amount of concurrently available reinforcement. The persistence of high rates of responding in Component A does seem to be an effect of behavioral history, but because of the complexity of the responding that that led to the response-rate differences in history testing, these effects of history not lend themselves to easy analysis. Nevin and Grace (2000) noted this type of problem in their discussion of one apparent disagreement between momentum theory and preference, stating, "In general, it may prove difficult to compare resistance to change between performances differing in temporal pattern or topography of responding, and apparent failures of agreement with preference may arise for this reason" (Nevin \& Grace, 2000, p. 84).

The second procedural feature that must be considered in interpreting the results of Experiments 1 and 2 is the change in the stimuli in each component from history building to testing. This change in stimuli included both the number of keylights present and the frequency and distribution of reinforcement in each component. These differences provide a further challenge to an analysis of history effects because, like the difference in the number of operants, these changes could have had different effects across the two components.

One way in which the stimuli correlated with the past contingencies were different from history building to testing was in the number of keylights illuminated within a component. The change from two keylights to one may have had a greater effect on responding in Component A than B. In Component A, there was more reinforcement, and therefore, more responding on the center key, and the center-keylight stimulus was not present in the history-testing condition. In Component B, there was less reinforcement, and therefore, less responding on the center key, and the center-keylight stimulus was not present in the history-testing condition. It is possible that, given the different rates of reinforcement related to the center keys across components, the combination of stimuli that had a discriminative function on responding was different in each component, and thus, the effect of behavioral history could be greater in Component B than A. As Sidman (1960) has said, "historical factors will intrude into an investigation to the extent that the stimuli in the experimental situation resemble those of past contingencies" (p. 386). It is possible that in Component B, the experimental situation of the test condition resembled to a greater degree the past contingencies from Component B than A.

Another way in which the stimuli correlated with the past contingencies were different from history building to testing was in the frequency and distribution of the IRI. Like the change in the number of stimuli, this difference varied across components. In the case of IRIs, the overall frequency of reinforcement delivery was greater in Component A, and so the change to FI $40 \mathrm{~s}$ in the history-testing condition may have resembled the history-building conditions less in Component A then B. In a study of effects of reinforcement history across two components of a multiple schedule, Okouchi and Lattal (2006) showed that behavioral persistence was greater in a component that had similar mean IRIs, than in a component that had different mean IRIs in history-building and -testing conditions. Although the present experiments were not designed to determine the function of these particular stimulus changes, potential effects of differential changes across components should be considered in the analysis of responding in history testing. Previous research has shown that it can be difficult to predict what features of a stimulus array will come to control responding in an individual subject (cf. Reynolds, 1961) but that the topography of stimulus control can be affected by the amount of reinforcement gained in presence of particular features (cf. Dube \& McIlvane, 1997). These findings can be taken to encourage caution in interpreting the degree to which the stimulus conditions were functionally similar across history building and history testing and the degree to which this similarity was equal across components.

This difficulty in determining stimulus control topography should be taken into account when considering the complicating third procedural feature of Experiments 1 and 2: the consequent function of the changes in reinforcement frequency and periodicity. Studies of behavioral momentum have found in many cases that different results occur with different disruptors (cf. Harper \& McLean, 1992). Disruptors that affect the degree of deprivation, such as feeding the subject prior to the session, have yielded response rate changes that are consistent with the predictions of behavioral momentum theory. Contrarily, disruptors that alter directly the rate of reinforcement obtained in test sessions, such as testing in EXT or another schedule of reinforcement, frequently have yielded response rate changes that are inconsistent with the predictions of behavioral momentum theory (e.g., Harper \& McLean, 1992; McLean \& Blampied, 1995). Some studies that have used a different schedule of reinforcement in testing have found that response rate was less resistant to disruption in a component with a richer schedule of 
reinforcement in the training condition. To account for this difference, Nevin \& Grace (2005) included a quantitative factor for the generalization decrement caused by the greater amount of change in reinforcement rate during testing in the formerly richer component, maintaining their argument that behavioral resistance to disruption was the product of the contingency between contextual stimuli and historic rate of reinforcement, and not a function of the change in reinforcement rate contacted in the testing condition. With respect to the results of Experiments 1 and 2 in the current report, the important consideration is that changes in the rate of reinforcement in testing conditions have been shown to affect differentially resistance to change of rich and lean components of multiple schedules, regardless of whether these effects result from a stimulus-reinforcer or a stimulus-response-reinforcer contingency.

The primary purpose of this study was to attempt to disentangle effects of historical response- and reinforcementrate on subsequent responding. Specifically, the question was: to what degree does historical response rate per se affect persistence in the face of changed contingencies? In previous studies this question has been addressed with schedules of reinforcement that directly controlled response rate, while reinforcement rate was equal across two groups or components of a multiple schedule. These studies found that low-rate responding resulted in more persistence, but this was confounded with rate-controlling contingencies such as DRL, DRH, or FR schedules. The present study attempted to generate different response rates across components by manipulating the context of reinforcement, rather than contingencies that specified response rates. Several problems were identified with the procedures used to manipulate the context of reinforcement in Experiments 1 and 2, including most importantly, the failure to establish sufficiently large differences in response rate across components in all pigeons. The difficulties encountered in Experiments 1 and 2 limited the degree to which their results bear on the question of effects of historic response rate on behavioral persistence. In Experiment 3 yet a different procedure was used to generate response-rate differences with the same schedule of reinforcement across two components: the context of reinforcement was not defined by the availability of reinforcement for pecking an alternative key, but by the proportion of response-independent food delivery. 


\section{Chapter 5: Experiment 3}

In Experiment 3 the context of reinforcement for side-key responses was not provided by the availability of an alternative response, but by the presentation of response-independent food in one component. The procedure used in Experiment 3 was based on the finding that response rate can be decreased by increasing the proportion of responseindependent food within a component (Lattal 1974). This proportion of response-independent food can be increased while the interfood interval is held constant between components (cf. Nevin et al., 1990, Experiment 1, Conditions 5 and 6). Although there was no alternative response in Experiment 3, response-independent food in one component provided an alternative source of reinforcement in one of two multiple-schedule components. The purpose of including response-independent food was to decrease response rate on one side key, while maintaining identical schedules of food delivery across the two side keys. The history-building condition in Experiment 3 controlled for two potential problems that were present in the first two experiments: (a) a change in stimulus conditions from two response keys to one response key, and (b) differential changes in the overall amount of reinforcement between the two components when pigeons went from the history-building to the history-testing conditions. In Experiment 3 these potential problems were controlled for by the presentation of the same stimuli in history building and testing conditions, and the same total amount of reinforcement in both components. To the extent that historical response rate per se, independent of reinforcement schedule factors, controls future response rate in the face of changed contingencies, response-rate differences should persist under the changed contingencies.

\section{Method}

\section{Subjects and Apparatus}

The subjects were four experimentally naive male White Carneau pigeons maintained at $80 \%$ of their ad libitum body weight. The pigeons were housed separately in a colony room with continuous access to water and digestive grit. The pigeons were not the same pigeons used in the Experiments 1 and 2. The apparatus was identical to that used in Experiment 1.

\section{Procedure}

Preliminary Training. Preliminary training sessions for Experiment 3 were identical to those for Experiment 1.

Multiple Schedule. Many procedural details of the multiple schedule sessions in Experiment 3 were the same as in Experiment 1. These identical features include the number of sequences and components, the frequency and duration of sessions, the presession blackout, and the ICIs. Distributions for all variable (VI and VT) schedules were generated according to the progression described by Fleshler and Hoffman (1962). As in Experiment 1, a history-building condition which was designed to build response-rate differences was followed by a history-testing condition. Table 12 shows the number of sessions of each condition for each pigeon.

Table 12: Number of Sessions for Each Pigeon in Experiment 3

\begin{tabular}{cccc}
\hline Pigeon & Pretraining & History Building & History Testing \\
\hline 609 & 15 & 62 & 73 \\
929 & 15 & 60 & 73 \\
567 & 15 & 70 & 70 \\
924 & 13 & 60 & 80 \\
\hline
\end{tabular}

History Building. Once magazine training, key-peck shaping, and schedule leaning were complete, Pigeons $609,929,567$, and 924 were exposed to the history-building condition. The history building condition for this experiment was composed of a multiple (mixed VI VT) / (VI) schedule of reinforcement. One side key was illuminated in each component of history building. Components were $60 \mathrm{~s}$ in duration. The upper portion of Figure 21 illustrates the history-building condition. The inclusion of response-independent food in Component A was 
designed to engender lower response rates on the side key than on the side key in Component B (cf. Lattal, 1974), while maintaining identical schedules of food delivery across the two components.

The upper portion of Figure 21 illustrates the history-building condition at the terminal schedule values. Stimulusposition-schedule combinations for each pigeon are detailed in Table 13. The arrangement for Pigeon 609 provides an example: In Component A, the left-white key was illuminated. At the start of the first component, and after each food delivery, the computer determined, according to a preprogrammed probability, whether or not a response would be required for the next food delivery. Intervals after which food was delivered only after a keypeck, and intervals after which food was delivered regardless of keypecking hereafter will be referred to as VI trials, and VT trials, respectively. A single Fleshler-Hoffman distribution was used in the scheduling of both VI and VT trials. Component A is illustrated in the top row of Figure 21. In Component B, VI-30-s reinforcement was effected on the right-white side key (second row of Figure 21). The VT trials constituted a contextual feature designed to decrease the rate of responding in Component A.

\title{
Experiment 3
}

\section{History Building}

\author{
Component $\mathrm{A}$ \\ Component B

\section{History Testing}
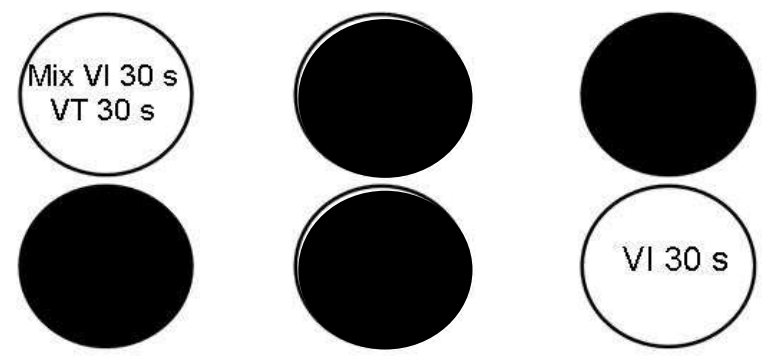

Component A
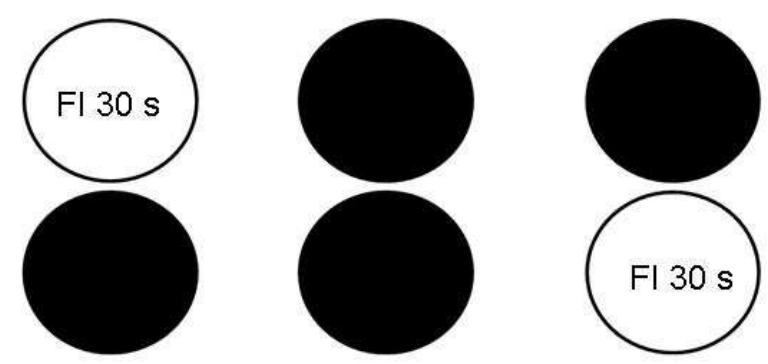

Figure 21. Illustration of history-building and history-testing conditions in Experiment 3.

In the first sessions of history building the probability of a response-independent trial in Component B was adjusted individually for each pigeon in an attempt to maximize the difference in response rates between the side keys in the two components, while still maintaining responding in the mixed VI VT component. Appendix D shows the number of sessions conducted with each pigeon at each probability level. Regardless of the proportion of VT food deliveries, the mean scheduled interfood interval on each of the two side keys was always $30 \mathrm{~s}$. As in Experiment 1, the historybuilding condition did not end for a pigeon until there were at least 20 consecutive sessions at the terminal values (and a minimum total of 60 sessions had occurred). 
Table 13: Stimulus-Position-Component Combinations for Each Pigeon in Experiment 3

\begin{tabular}{ccc}
\hline Pigeon & Component A & Component B \\
\hline 609 & White (Left) & White (Right) \\
929 & White (Right) & White (Left) \\
567 & White (Left) & White (Right) \\
924 & White (Right) & White (Left) \\
\hline
\end{tabular}

Note. The center key was dark and inoperative in both components.

History Testing. A history-testing condition followed history building. As illustrated in the lower portion of Figure 21, history testing consisted of a two-component multiple FI 30-s FI 30-s schedule of reinforcement, and the stimuli presented on the side keys were identical to those in the history-building condition. These FI values were equal to the mean interfood intervals in history building.

Following the 90-s presession blackout, two FI components alternated in the same manner as in history building; thus, components were approximately $60 \mathrm{~s}$ in duration. Each component ended after the end of the delivery of the second reinforcer. The history-testing condition was conducted: (a) until a minimum of 70 sessions were completed and (b) for the last 3 (minimum) there was a consistent difference in response rate across components (e.g. response rate on the left component was higher for three consecutive days), or (c) for a maximum of 80 sessions.

$$
\text { Results }
$$

\section{History Building}

Response Rate. Table14 shows the mean and range response rate averaged across the last 20 sessions of history building for each component. Response rate was greater for each pigeon in Component B than Component A. These differences are summarized in Table 14 as proportional response rates and are also displayed in Figure 22. Response-rate differences across the components were large in all pigeons, ranging from 0.59 to $0.79(\mathrm{M}=0.72)$.

Table 14: Mean (and Range) Response Rate per Min over the Last Twenty Sessions of the History-Building Condition in Experiment 3

\begin{tabular}{cccc}
\hline & Component A & Component B & \\
\cline { 2 - 3 } Subject & Mixed VI30-VT30 & VI30 & $\begin{array}{c}\text { Proportional } \\
\text { Response Rate }\end{array}$ \\
\hline 609 & $35(11-75)$ & $120(103-133)$ & 0.77 \\
929 & $66(30-90)$ & $94(77-107)$ & 0.59 \\
567 & $30(17-57)$ & $113(103-126)$ & 0.79 \\
924 & $34(17-50)$ & $98(32-116)$ & 0.74 \\
\hline
\end{tabular}

\footnotetext{
${ }^{a}$ Proportional Response Rate $=\mathrm{B} /(\mathrm{B}+\mathrm{A})$.
} 


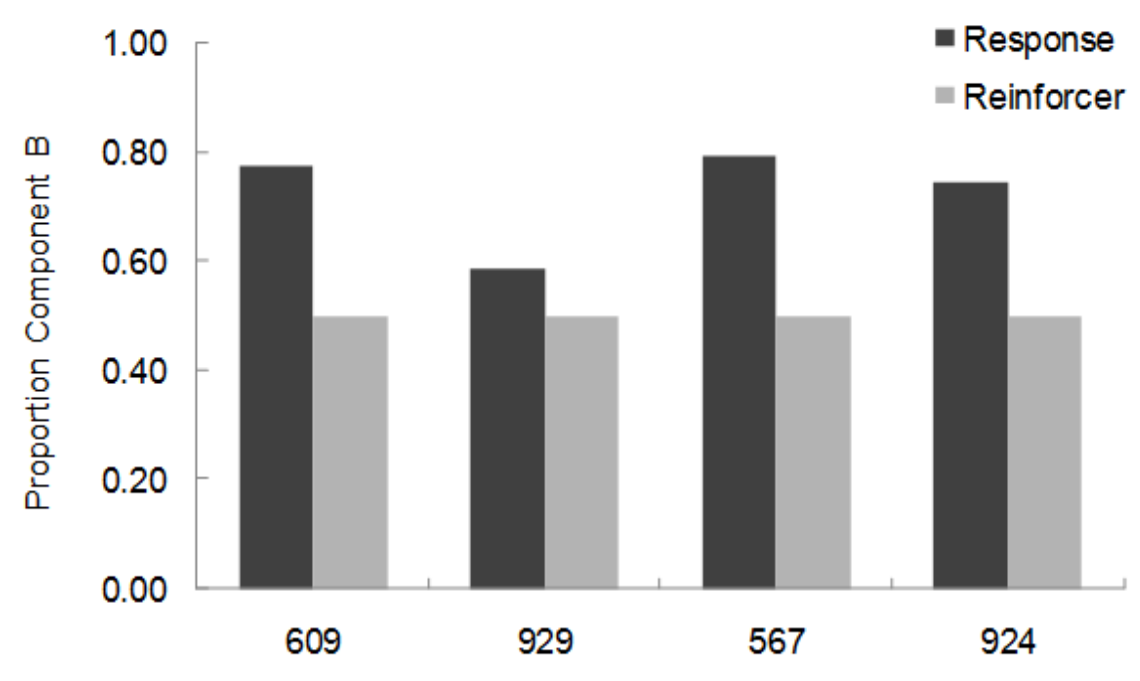

Pigeon

Figure 22. Proportional response rates and reinforcement rates in the history-building condition of Experiment 3. The quotient of the response rate (dark grey) and reinforcement rate (light grey) in Component $\mathrm{B}$ divided by the sum the response rates in both components.

Reinforcer Rate. Mean rates of response-dependent and response-independent reinforcers across the two components are shown in Table 15. Reinforcer rates in Component B as a proportion of reinforcer rates in both components in are shown in Table 15 and Figure 22. The rate of food delivery was equal across the two components (as indicated by values of 0.50 in the rightmost column of Table 15).

Table 15: Mean (and Range) Reinforcement Rate per Hour over the Last Twenty Sessions of the History-Building Condition in Experiment 3

\begin{tabular}{|c|c|c|c|c|c|}
\hline \multirow[b]{2}{*}{ Pigeon } & \multicolumn{2}{|c|}{ Component A } & \multirow{2}{*}{$\begin{array}{c}\text { Component B } \\
\text { VI30 }\end{array}$} & \multicolumn{2}{|c|}{ Proportional SR Rate } \\
\hline & VI30 & VT30 & & $\begin{array}{l}\text { Response } \\
\text { Dep. Food }\end{array}$ & All Food \\
\hline 609 & $0(0-0)$ & $116(116-116)$ & $115(108-116)$ & 1.00 & 0.50 \\
\hline 929 & $0(0-0)$ & $117(110-140)$ & $116(109-135)$ & 1.00 & 0.50 \\
\hline 567 & $6(4-8)$ & $110(98-113)$ & $115(109-116)$ & 0.95 & 0.50 \\
\hline 924 & $28(19-30)$ & $83(45-90)$ & $110(34-116)$ & 0.80 & 0.50 \\
\hline
\end{tabular}

${ }^{a}$ Proportional Reinforcement Rate (Response Dependent Food) $=$ VI reinforcers B / (VI reinforcers B + VI reinforcers A). ${ }^{b}$ Proportional Reinforcement Rate $($ All Food $)=$ VI + VT reinforcers B / (VI reinforcers B + VT reinforcers B + VI reinforcers A).

History Testing

Response Rate. Response rate data for each pigeon in Experiment 3 are shown in Figure 23. Each data point represents the mean response rate over a block of 10 sessions (except in pigeons for which the total number of sessions was not a multiple of 10. In these cases, the last data point is the mean of the last 13 sessions; see figure caption for details). The left portion of each graph displays response rates in each component in the last twenty 
sessions of history building, and the right portion displays the response rates in history testing. In general, the mean rate of responding in the first 10 sessions increased in Component $\mathrm{A}$, and decreased in Component $\mathrm{B}$, relative to the history-building condition. Response rates in the two components varied widely across pigeons. Figure 24 shows the $\log$ ratio of response rates in the first session of history testing as a proportion of the mean response rate in the same component from the last 20 sessions of history building. In Pigeons 609 and 567, response rate increased in Component A relative to response rate during history building, and decreased in Component B. In the other two pigeons, the opposite relation was seen: response rate decreased in Component A and increased in Component B; but the magnitude of these changes was small.

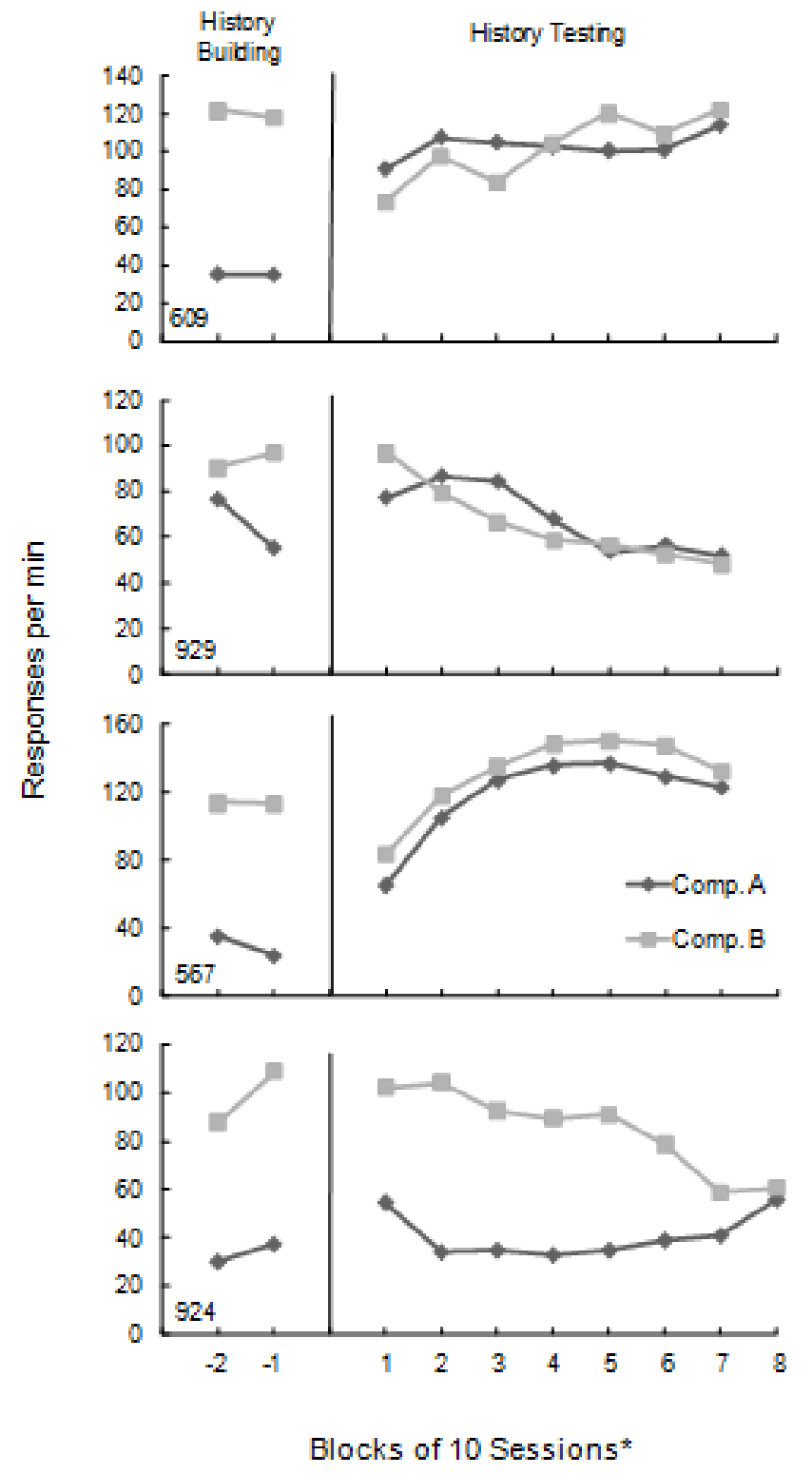

Figure 23. Response rate data for each pigeon in Experiment 3. The left portion of each graph displays response rates in the last twenty sessions of history building, and the right portion displays the response rates in the two components in history testing.

*Each data point represents the mean response rate over a block of 10 sessions, with the following exceptions: For both 609 and 929, the last data point shows the mean of the last 13 sessions. These exceptions apply to all figures for Experiment 3 that display data in 10-session blocks. 


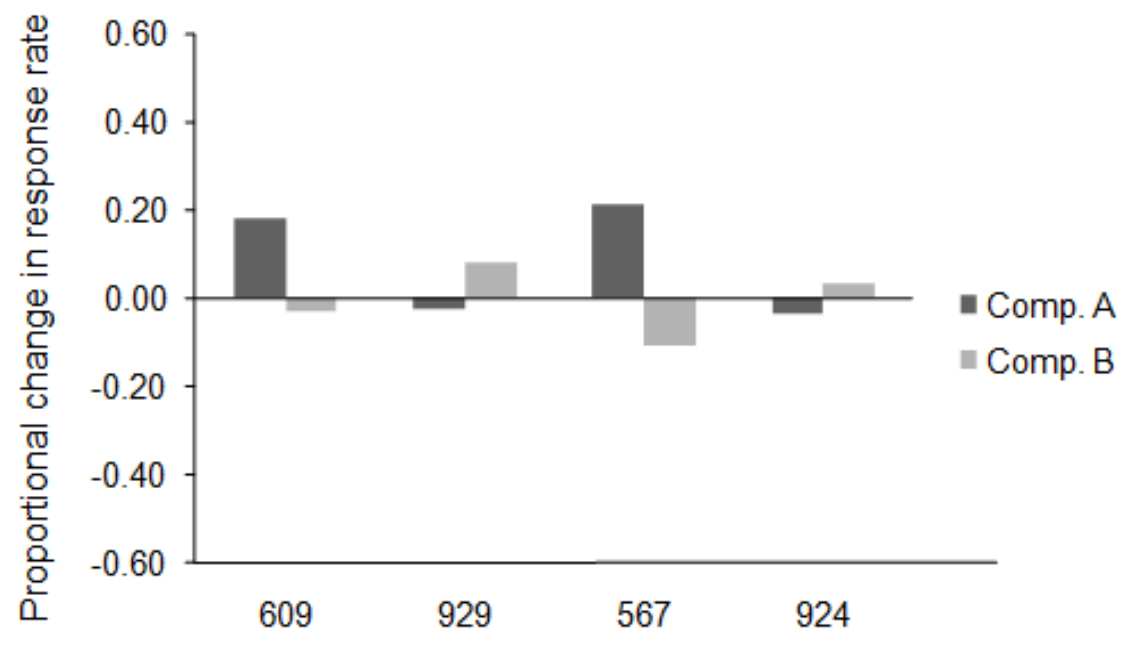

Pigeon

Figure 24. Experiment 3: Log ratio of response rates in the first session of history testing as a proportion of the mean response rate in the same component from the last 20 sessions of history building.

Response-Rate Differences. Figure 25 shows the difference in response rates in Components A and B, as a proportion of the sum of the response rates in both components. These data allow a comparison of response-rate differences between components, without the influence of differences in the level of responding, which varied widely across pigeons. This comparison of response rates in 10-session blocks shows that throughout the historytesting condition, Pigeons 567 and 924 responded at a higher rate in Component B than A, though only in Pigeon 924 were differences in proportional response rates similar to those in the history-building condition. 


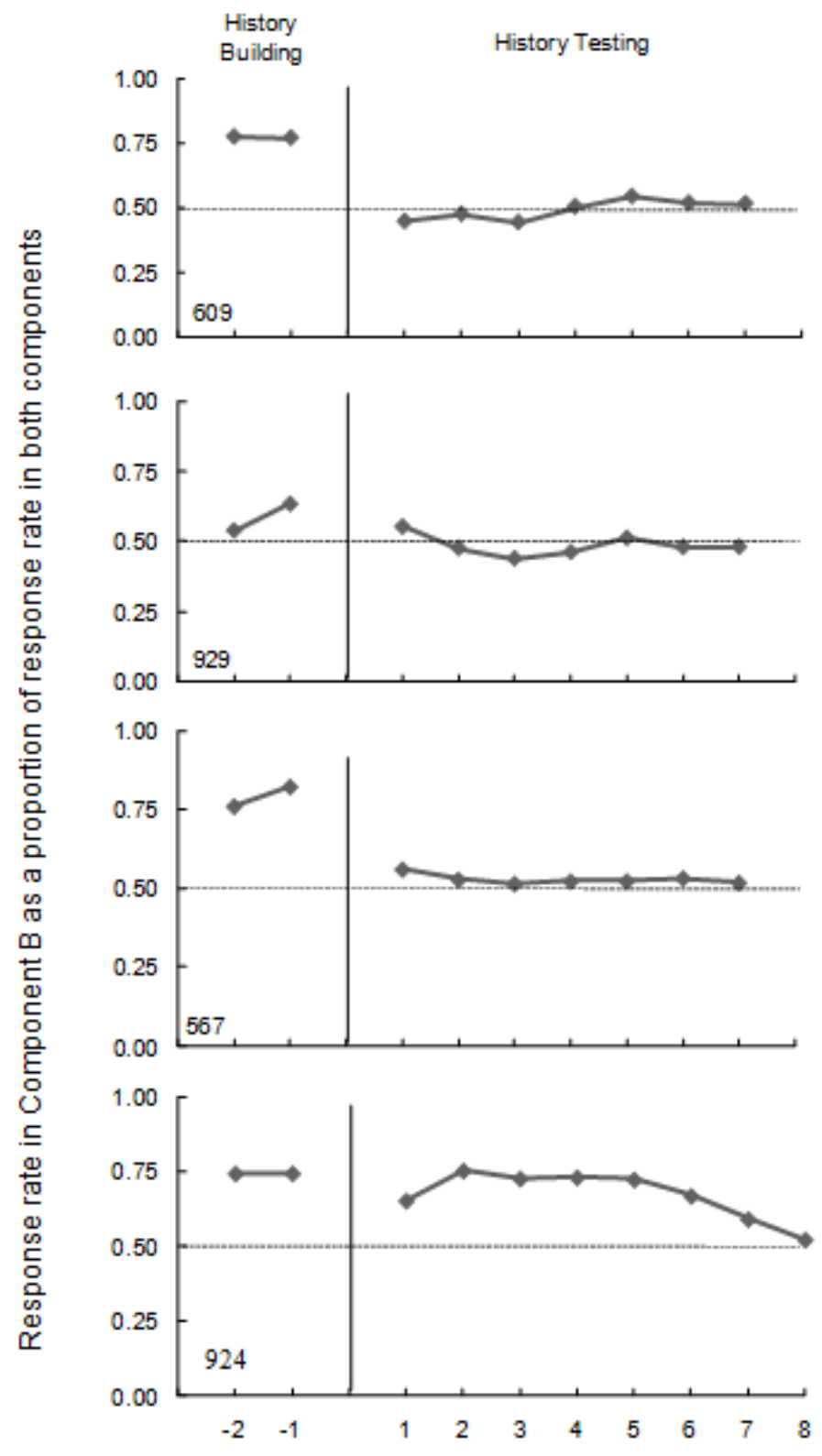

Blocks of 10 Sessions*

Figure 25. Experiment 3: difference in response rates in Components A and B. In the left portion of each panel are the response rates on the side key in Component B as a proportion of side key responding in both components and the response rates for overall responding in Component $\mathrm{B}$ as a proportion of all responding in both components.

Rate of Change. The rationale for comparing rate of change of reponse rate across components was described in the Results section for Experiment 1. Figure 26 shows the log proportion of the response rate in each component of history testing. The amount of change at the end of 10 sessions was greater in Component A than Component B in each pigeon, and this difference between components in the magnitude of change from baseline persisted throughout history testing in two pigeons, 609, and 567. 


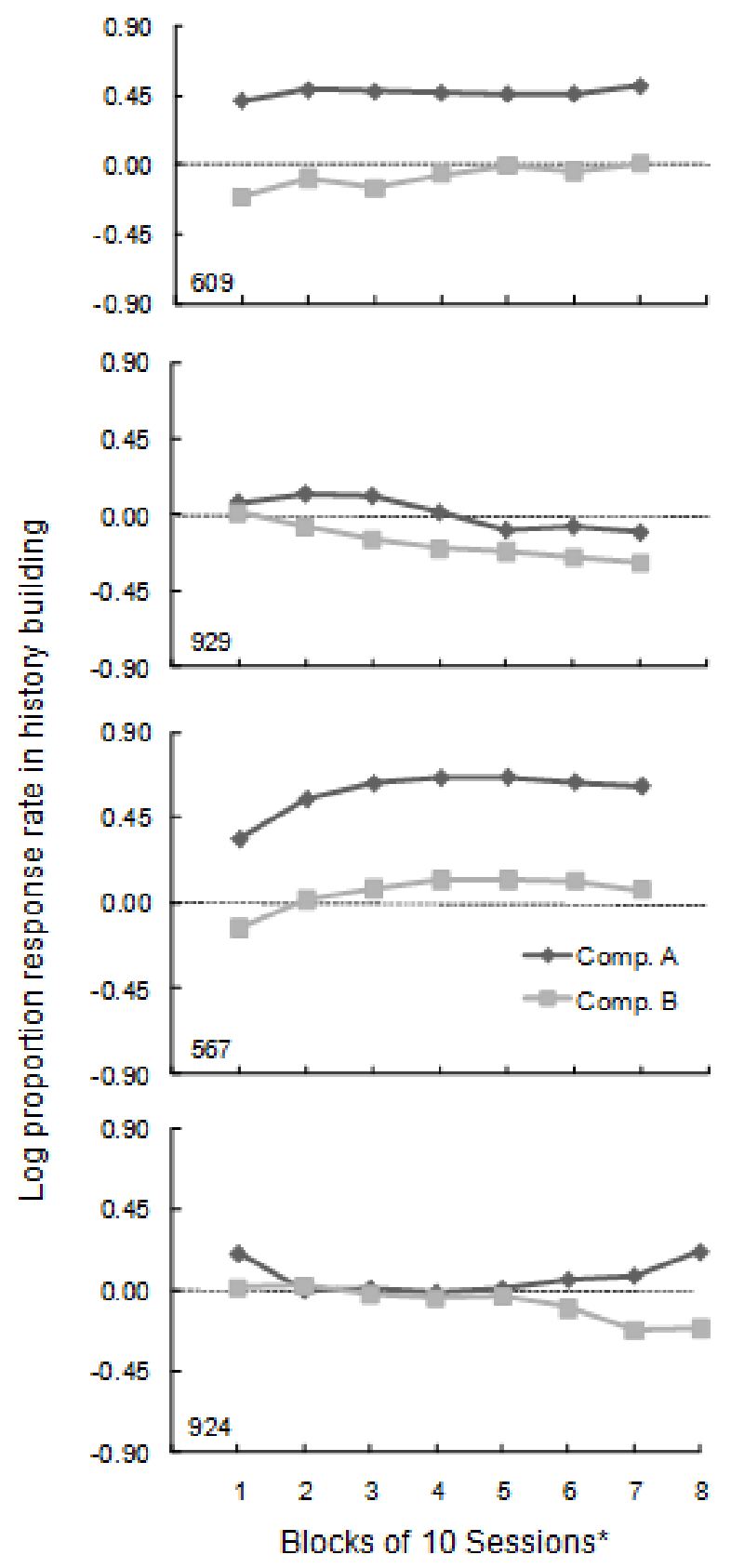

Figure 26. Experiment 3: log proportion of the response rate in each component of history testing. Proportions are calculated for each component by dividing response rate in history testing by the mean response rate on the side key in the final 20 sessions of history building, and then taking the $\log$ of this. Log-proportion data are presented in 10-session blocks. The slope of each line illustrates the degree to which response rate changed over the course of history testing. The level of the data, with respect to the ordinate, indicates the degree to which response rate is greater or less than it was in history building. A result of " 0 " on the ordinate would occur were response rate in history testing equal to response rate for the same component in history testing (i.e., equal response rates would yield a ratio of 1 , and the $\log$ of 1 is 0 ). 
Figures 27 and 28 allow a comparison of the rate of change in response rate in the two components. Figure 27 shows the slope of the log ratios of response rate for each pigeon in the first 20 sessions of testing (left panel) and for the all of the test sessions (right panel). In the first 20 sessions, proportional response rates changed more in Component A than in Component B in three of the pigeons (609, 567, and 924). Session-by-session response-rate data for the first 20 sessions are displayed in Figure 28. In all four pigeons, there was more overall change in response rate over the first 20 sessions than over the entire course of the testing condition.

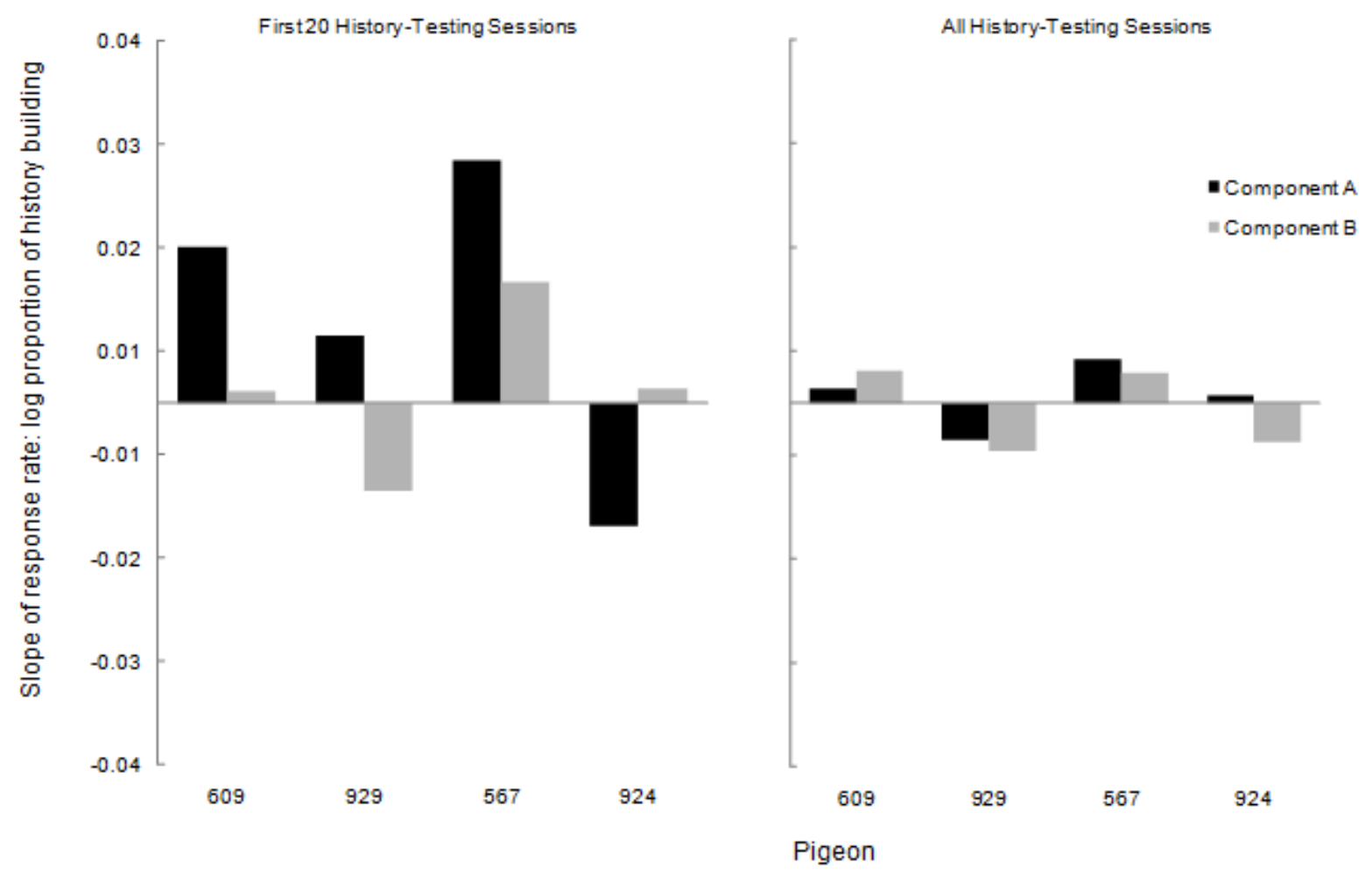

Figure 27. Experiment 3: slope of the log ratios of response rates on the side keys for each pigeon in the first 20 sessions of testing (left panel) and for the all of the test sessions (right panel). To calculate these slopes, the mean of the last 20 sessions of history building was included as the first data point, and individual session data were used for the history-testing condition. 


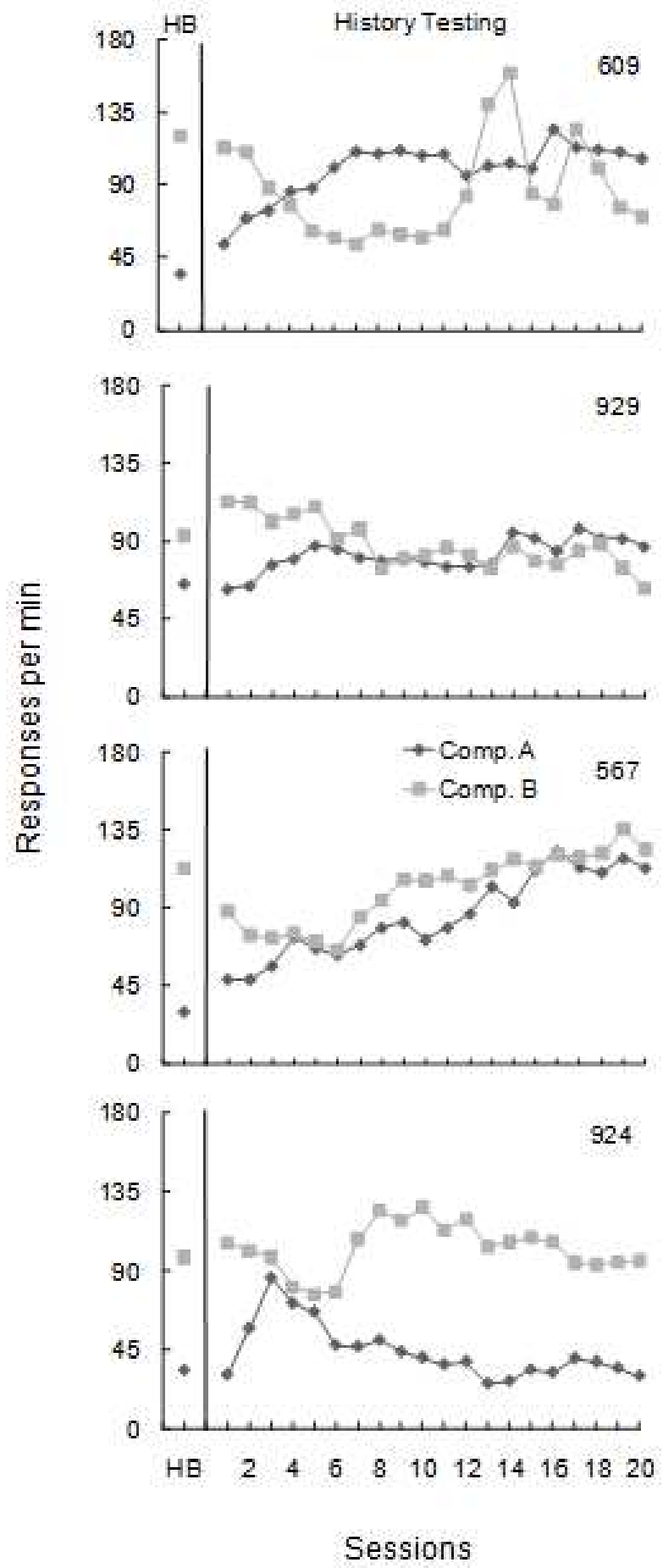

Figure 28. Experiment 3: session-by-session response-rate data for the first 20 sessions.

Pattern of Responding. As can be seen in Figure 29 positively accelerated responding was slow to develop in three of the four pigeons. This was especially the case in Pigeon 929, for which the 10-session mean QL never exceeded 0.29 in either component. In each pigeon the mean QL for the first 10 sessions was greater in Component A than B (though only slightly so in Pigeon 929). In both components the QL continued to increase after these first 
sessions. In three of the four pigeons the maximum 10-session mean QL in Component A was greater than or equal to that in Component B.
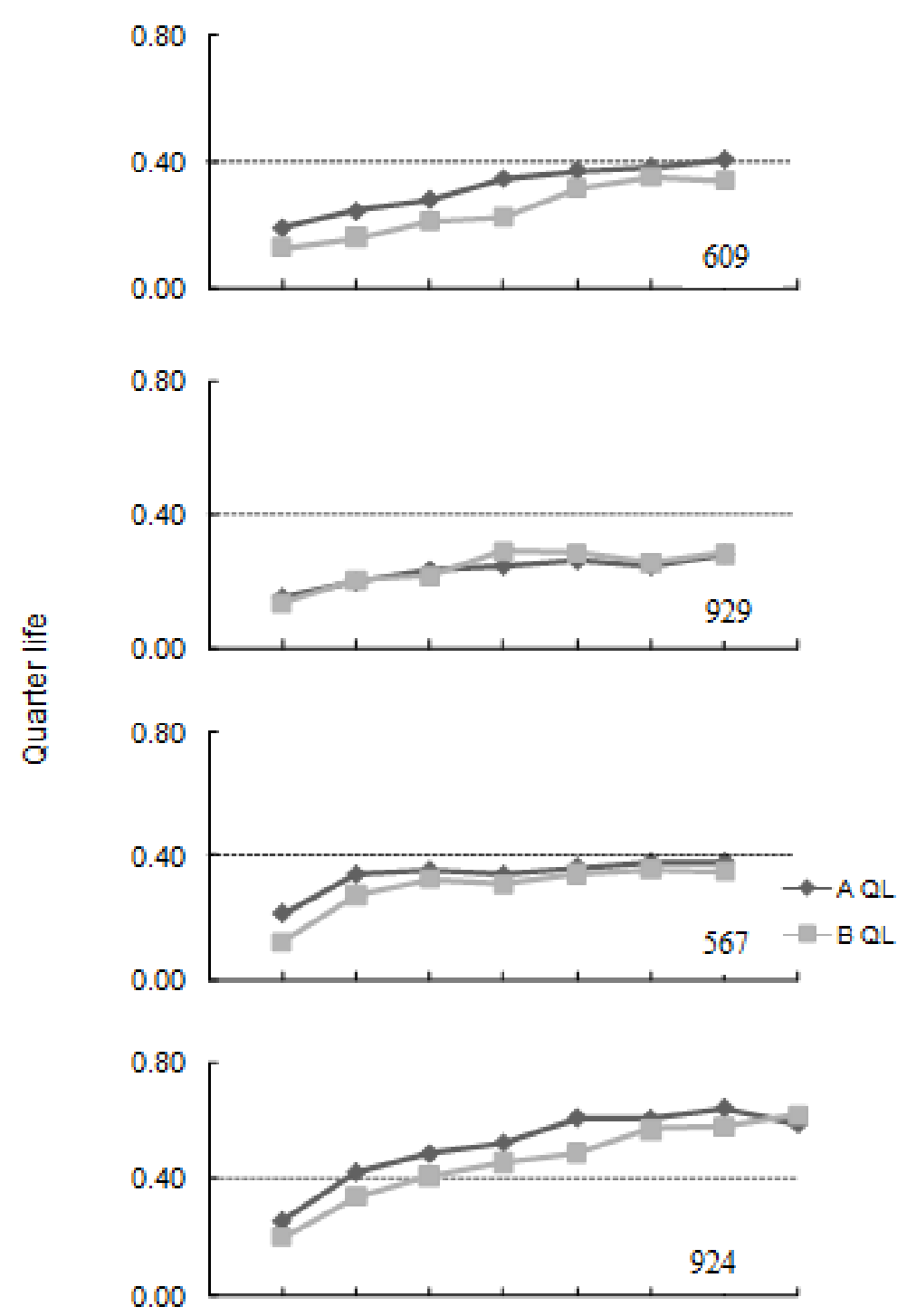

Blocks of 10 Sessions*

Figure 29. Experiment 3: mean QL in each component of the history-testing condition in 10session blocks.

Other Measures of Persistence. Measures shown in Table 16 were described in the Results section for Experiment 1. Table 16 allows a comparison across pigeons of measures of persistence in history testing. In each pigeon, response rates in history testing met stability criteria in both components within 20 sessions. Also in each pigeon, standard deviations in response rate were greater in component $\mathrm{B}$ than $\mathrm{A}$ in the first 10 sessions of history testing, and the rate of decrease in standard deviation was greater in component B than A. Finally, in three of the four pigeons, it took fewer sessions for QLs to increase to at least 0.40 for at least five consecutive days in Component A than B, and in the fourth pigeon, QLs never reached the 0.40 criterion. There is inconsistency between pigeons on (a) the number of sessions for which the response rate in Component $B$ is greater than that in Component A (B $>A /$ total test sessions), (b) the number of sessions until a change in the sign of the differences 
between response rate in $\mathrm{A}$ and $\mathrm{B}$, and (c) the number of sessions to convergence of response rate between the two components (10 Resp / min and 10\% Range).

Table 16: Measures of Persistence for Experiment 3

\begin{tabular}{|c|c|c|c|c|}
\hline \multirow[b]{2}{*}{ Measure of Persistence } & \multicolumn{4}{|c|}{ Subject } \\
\hline & 609 & 929 & 567 & 924 \\
\hline $\mathrm{B}>\mathrm{A} /$ total test sessions & $39 / 73$ & $28 / 73$ & $65 / 70$ & $77 / 80$ \\
\hline Sign change 1 & 4 & 8 & 16 & 70 \\
\hline Sign change 2 & 13 & 9 & 17 & 71 \\
\hline $10 \operatorname{Resp} / \mathrm{min}$ & 64 & 47 & $>70$ & 74 \\
\hline $10 \%$ Range & 31 & 10 & 19 & 74 \\
\hline $10 \%$ terminal: A & 11 & 50 & 23 & $>80$ \\
\hline $10 \%$ terminal: $\mathrm{B}$ & 41 & 61 & 20 & 78 \\
\hline 1st Stable (no. sessions) & $10(2)$ & $11(4)$ & $19(38)$ & $16(1)$ \\
\hline 2nd Stable (no. sessions) & $19(1)$ & $18(3)$ & $59(9)$ & $20(1)$ \\
\hline SD: A (1st 10 sessions) & 20.26 & 8.04 & 11.54 & 16.36 \\
\hline SD: B (1st 10 sessions) & 22.48 & 14.11 & 14.50 & 18.44 \\
\hline $\begin{array}{l}\text { Slope of change in SD: A } \\
\text { (10 session blocks) }\end{array}$ & -1.23 & -0.90 & -0.48 & -0.38 \\
\hline $\begin{array}{l}\text { Slope of change in SD: B } \\
\text { (10 session blocks) }\end{array}$ & -3.07 & -1.40 & -0.50 & -0.62 \\
\hline $\begin{array}{l}\mathrm{QL}>=0.40: \mathrm{A} \\
(1 \mathrm{st} \text { instance })\end{array}$ & 32 & $>73$ & 20 & 14 \\
\hline $\begin{array}{l}\text { QL > = 0.40: B } \\
\text { (1st instance) }\end{array}$ & 50 & $>73$ & 21 & 19 \\
\hline $\begin{array}{l}\mathrm{QL}>=0.40: \mathrm{A} \\
(5 \text { consec days })\end{array}$ & 69 & $>73$ & 60 & 22 \\
\hline $\begin{array}{l}\mathrm{QL}>=0.40: \mathrm{B} \\
(5 \text { conseq days })\end{array}$ & $>73$ & $>73$ & $>70$ & 30 \\
\hline
\end{tabular}




\section{Discussion}

In Experiment 3 contextual differences in schedules of reinforcement across two components of a multiple schedule were programmed in attempt to generate behavioral histories of high- and low-rate responding, using equal schedules of reinforcement. Effects of these two behavioral histories on subsequent responding under novel contingencies were examined in a history-testing condition. Although most of the food deliveries (75-100\%) in one component were provided independently of responding, mean IRIs across the two components were equal. The inclusion of response-independent food in one component resulted in relatively low response rates in the same component; as a result, the differences in response rates in the two components were large.

There was no evidence that response rates in the low-rate component (Component A) were more resistant to change than response rates in the high-rate component (Component B). Effects of behavioral history were evident, however, in several features of responding in the history-testing condition: 1) between-component response-rate differences of greater than 10\% that persisted for 10, 19, 31, and 74 sessions in Pigeons 929, 567, 609, and 924, respectively; 2) an initially greater rate of change in response rate in Component A than Component B in all four pigeons; 3) betweencomponent differences in rate of change of response rate over the course of history testing in all pigeons; and 4) a greater rate of increase in QL in Component A than B over the first 10 sessions in all pigeons, and generally larger QLs in Component A in three of four pigeons. Although the history effects that were found in this study varied across pigeons, the historical differences between the contexts of the two components did have some lasting effects on responding.

Previous studies of behavioral history that have sought to generate response-rate differences with equal rates of reinforcement have typically used rate-controlling schedules (such as DRL, DRH, and FR) in their history-building components. These contingencies may have confounded the effects of historical low- vs. high-rate responding with the schedules used to generate these rates. In Experiments 1 and 2 responding on an alternative response key was used in an attempt to disentangle effects of historic response-rate differences from the effects of historic ratecontrolling contingencies. The current experiment was an attempt to generate response-rate differences across two components with equal rates of food delivery while avoiding some of the problems engendered by three types of changes that pigeons encountered in Experiments 1 and 2 when they were transitioned from history building to history testing. These changes were in: (a) the number of available operants, (b) the stimulus conditions, and (c) the overall rate of reinforcement.

Experiment 3 was designed to eliminate or minimize procedural features of Experiments 1 and 2 that were found to be potentially problematic. In Experiment 3, there was only one response key in each component - no reinforcement was arranged for any response other than pecking the side keys. It is possible, however, that the VT food in Component A led to an increase of some other response through adventitious reinforcement (Skinner, 1948; Staddon \& Simmelhag, 1971). If superstitious responding did develop in the component with VT food, it seems it would not have as potent an effect on subsequent responding as would responding that had been reinforced for pecking a separate key. One reason for this supposition is that superstitious responding is known to be transient, with response topographies shifting over the course of exposure to reinforcement schedules (Skinner, 1948, Staddon \&

Simmelhag, 1971). Another reason for the supposition is that when adventitious reinforcement maintains a response, it is not always delivered immediately after that response, and responding maintained by delayed reinforcement is less resistant to change (Bell, 1999; Grace, Schwendiman \& Nevin, 1998). It follows that effects on responding resulting from a history of superstitious behavior maintained by adventitious reinforcement likely would be less durable than effects resulting from a history of responding maintained by immediate reinforcement on a separate response key. The use of one response key in each component of history building in Experiment 3, therefore, handled the problem of the availability of two operants in each component in Experiments 1 and 2.

In Experiment 3 the difference in stimulus conditions between history-building and history-testing conditions were minimal. Whereas in Experiments 1 and 2 there were changes in the number of available operants, the number of keylights, the overall mean IRI, and the periodicity of reinforcement in each component, in Experiment 3 there were changes only in periodicity and response dependency. Changes in periodicity from VI to FI have not been shown to diminish behavioral history effects; on the contrary, as discussed earlier, FI schedules tend to extend the lifespan of history effects (Freeman \& Lattal, 1992; Poling et al., 1980). The change from response-independent to responsedependent schedules of food presentation was likely the most salient stimulus change the pigeons experienced in the first testing sessions of Experiment 2. These minimal stimulus changes were less likely to diminish history effects in Experiment 3 than the changes in stimulus conditions in Experiments 1 and 2. 
Finally, in Experiment 3, there was no change in the overall rate of reinforcement in either component between history building and history testing. This lack of change in IRI from history building to history testing contrasts with the large differences in overall rate of reinforcement in each component of history building in Experiments 1 and 2, relative to rate of reinforcement in history testing. The preservation of reinforcement rates from history building to history testing in Experiment 3, therefore, circumvented the problem of changes in the overall rate of reinforcement in Experiments 1 and 2.

The primary purpose of this experiment was to investigate effects of historically different rates of responding generated by equal schedules of reinforcement. The history-building condition in Experiment 3 yielded responserate differences in two components of a multiple schedule with equal rates of reinforcement, and these response-rate differences allowed an evaluation of the contributions of response-rate differences on responding under a subsequent history-testing condition.

Contrary to studies that have found lower-rate responding to be more resistant to change (Blackman, 1968; Metzger, 1992; Lattal, 1989; Nevin, 1974, Experiment 5; Nevin et al., 2001; Tatham et al., 1993; Urbain et al., 1978), the results of the present experiment did not support these findings. Contrarily, several measures indicated lower-rate responding (Component A) to be less persistent than higher-rate responding (Component B). For example, the slope of the $\log$ ratio of response rate in history testing to response rate in history building was steeper in Component A than B in three of four pigeons. This steeper slope indicates less resistance to change in the component with lowerrate responding. Additionally, positively accelerated responding developed more rapidly in Component A than B in the same three pigeons, and in the fourth pigeon scalloped response patterns were never robust over the course of the 73-session history-testing condition. Quicker acquisition of these response patterns indicate earlier control by the FI contingencies, further supporting the conclusion that lower-rate responding was less resistant to the history-testing contingencies. These findings of similar resistance to change of responding maintained by equal rates of reinforcement align with those of Nevin et al. (1990, Experiment 1, Conditions 5 and 6). Nevin et al. compared resistance to change across two components with equal rates of food delivery. In concert with Nevin et al. (1990, Experiment 1, Conditions 5 and 6), in the present experiment, in Component A low-rate responding was generated by a proportion of response-independent food, and in Component B higher-rate responding was generated with VI reinforcement. In contrast to the findings of Nevin et al. (1990), in the current study response rates increased in history testing in the component with VT food. This difference may be related to procedural differences between the two studies. The most important difference between the procedures in Nevin et al. (1990) and the present study was the type of manipulation used in the testing conditions. In Experiment 3, the testing condition consisted of (a) a change in the in the strength of the contingency in Component A, specifically an increase in response-contingent food proportions from $0-25 \%$ of all food deliveries to $100 \%$ of all food deliveries, and (b) a change in the periodicity of reinforcement in both components. With the exception of their resistance to extinction test, Nevin et al. (1990) did not change the parameters of reinforcement; and so in those test conditions they manipulated neither contingency nor periodicity of reinforcement. Although the results from the history-building condition of Experiment 3 are consistent with those of Nevin et al. (1990), they did not replicate findings from studies that have suggested that when reinforcer rates are equated, lower-rate responding is more resistant to change. The common finding that lowrate responding is more resistant to change may be more directly a function of the particular schedules used, and not of response rates per se. That is, differences in resisitance to change of behavior are related to the contingencies on response rate, and not to the response rates themselves.

Although effects of behavioral history did persist for each pigeon through some portion of the history-testing condition in Experiment 3, as in Experiments 1 and 2, there are clearly inconsistencies in mechanisms that controlled responding within and across pigeons. The General Discussion will include a treatment of the literature related to the differential resistance to change of high and low response rates, and in light of this literature will address data from the three experiments entailed in the current report. 


\section{Chapter 6: General Discussion}

The investigative lens of the experimental analysis of behavior (EAB) commonly is focused on effects of current contingencies on responding. In most research in this tradition, there is an attempt to minimize effects of prior experience, allowing the isolation of variables manipulated in real time during experimental sessions. Conditions are held constant until behavior approaches asymptote, and subsequent conditions are designed either as replications or points of comparison. This form of inquiry into variables affecting steady states of responding has been fruitful. An analysis of factors that influence behavior as it passes between these steady states is also important; these are known as transition states. The study of transition states includes the analysis of the rate of change of behavior, and the determinants of this rate of change. Nevin (1974) advanced the understanding of behavior with his position that response strength is best expressed as resistance to change. Subsequent research on resistance to change has flourished. Another approach to the study of transition states is seen in the behavioral history literature. Early researchers who examined effects of behavioral history were interested in individual differences in human schedule performance (e.g., Holland, 1958). In these studies, subjects typically were exposed to extended periods of one schedule of reinforcement, and then were tested on another schedule, typically FI. The rate and degree of change in response rate was taken to indicate the effect of historical schedules. These studies of effects of behavioral history allow an additional layer of complexity in the analysis of behavior. Indeed, if EAB is to build a framework of understanding that is relevant to human behavior at large, it is necessary to broaden the temporal lens and investigate effects of historical determinants of present behavior. The study of effects of behavioral history should be of particular importance to radical behaviorists, who view determinants of behavior to be the history of consequences. The study of behavioral history allows behavior analysts to take one step further into the past in their examination of effects of environmental events on behavior. A better understanding of effects of behavioral history on subsequent behavior may help researchers better understand and impact human learning, allowing educators, therapists, policy makers, and individuals to maximize efficacy and efficiency of behavior management approaches, and improve methods to help individuals transfer or generalize desirable behavior from one setting to another.

Nevin (1974; Nevin et al., 1990; Nevin \& Grace, 2000), his colleagues, and others have established that reinforcement parameters are related directly to resistance to change: greater rate or magnitude of reinforcement in a situation leads to less change when that behavior is met with some disruptor in a similar situation. Other factors that lead to increased resistance to change have not been as well established. Several studies have provided evidence that responding that occurs at a low rate is more resistant to change than responding that occurs at a high rate, when the schedules of reinforcement for the two responses are equal (e.g., Blackman, 1968; Doughty \& Lattal, 2001; Freeman \& Lattal, 1992; Lattal, 1989; Metzger, 1992; Nevin, 1974, Experiment 5; Nevin et al, 2001; Okouchi \& Lattal, 2006; Reed \& Doughty, 2005; Tatham et al., 1993; Urbain et al., 1978). A question raised by Lattal (1998) and others has been whether the more important relation in these cases is between behavioral persistence and historical response rates per se, or the contingencies that have generated the high and low response rates. Lattal (1998; Doughty \& Lattal, 2001) has argued that the contingencies that generate high and low response rates that are likely the critical factor in determining behavioral persistence, rather than the rate of responding itself. Most of the studies listed above used rate-controlling contingencies such as DRL, DRH, or FR to generate response-rate differences. The current study differed from these by attempting to generate response-rate differences using differences between two responses in the context of reinforcement for each response, while imposing identical contingencies on the two responses.

In the three experiments described in this report there was no evidence that lower-rate responding was more resistant to change. One barrier to interpretation was a difficulty with generating response-rate differences in Experiments 1 and 2, using procedures based on those of McDevitt and Williams (2003), who obtained proportional response-rate differences of 0.78 . Those researchers, however, did not report response rates, only proportional rates, and further investigation showed that this proportional response-rate difference was greater than that obtained in any other study reporting the use of similar procedures. In Experiment 3, a different strategy was used to generate response-rate differences, and in this case response-rate differences were obtained. Still, there was no evidence that lower-rate responding was more persistent.

The remainder of this discussion focuses on four topics. First is a discussion of procedures and results of the present experiments, in comparison to previous research, including (a) experimental design in behavioral history research, (b) resistance to change of low-rate responding, (c) the use of context to generate rate differences, and (d) the analysis of history effects. Second is a treatment of the broader theoretical framework: (a) implications for the study of behavioral history effects and resistance to change, and (b) relations between response rate and response unit. 
Third is a consideration of potential applied implications of (a) factors related to reinforcement context and persistence, and (b) factors related to discriminative stimulus function. And fourth is a presentation of conclusions and a review of implications of the present inquiry into historical response rates, context, and behavioral persistence.

\section{Procedures and Results of the Present Experiments}

\section{Experimental Design in Behavioral History Research}

Traditionally, studies of behavioral history have employed groups of subjects, and exposed each group to a different historical condition. After history building was completed, a history-testing condition was conducted. The primary dependent measure typically was rate or pattern of responding, and results were compared across groups. A significant disadvantage of using a group design in studies of behavioral history was that in many cases the level of behavior varied widely across subjects, so that differences between group mean were difficult to detect. Freeman and Lattal (1992) were innovative in their use of multiple schedules, which allowed them to build parallel histories within individual subjects with a multiple schedule history-building condition, and to test effects of these histories in a subsequent multiple-schedule history testing condition. Although the use of multiple schedules had long been used in studies of resistance to disruption (e.g., Nevin, 1974), it had not been demonstrated prior to Freeman and Lattal (1992) whether responding after a long history-testing condition would continue to be differentiated across components of a multiple schedule history-testing condition. As discussed in the Introduction section of the current paper, the methods used by Freeman and Lattal (1992) were successful, and allowed the comparison, in some cases, of small but consistent differences in responding within individual subjects. Several other studies from the behavioral history tradition have demonstrated that multiple schedules provide a useful method for comparing effects of different behavioral (e.g., Metzger, 1992; Ono \& Iwabuchi, 1997) or reinforcement histories (Okouchi \& Lattal, 2006). The procedures developed by Freeman and Lattal, extended conditions of history building and history testing under stimulus control in multiple schedules, provided the foundation for the design of the three experiments entailed in the current report.

\section{Resistance to Change of Low-Rate Responding}

Greater behavioral persistence or resistance to change as a function of low-response-rate history have been found both in typical behavioral history studies, with extended history-building and -testing conditions, and in typical resistance to change studies that have used the common disruption procedures, such as prefeeding, responseindependent food within the experimental test session, and EXT. Several studies, using a variety of procedures to generate response-rate differences and a variety of tests for behavioral persistence, have supported the finding that low-rate responding is more resistant to change. Low-rate responding generated by (a) IRT contingencies (Blackman,1968; da Silva et al., 2008; Freeman \& Lattal, 1992; Lattal, 1989; Metzger, 1992; Nevin, 1974, Exp 5; Okouchi \& Lattal, 2006; Tatham et al., 1993; Urbain et al., 1978), (b) interval schedules (Lattal et al., 1998, but response-rate differences were not obtained consistently; Nevin et al., 2001) (c) signaled reinforcement (Reed \& Doughty, 2005; Roberts, Tarpy, \& Lea, 1984), (d) larger or more complex response units (Doughty \& Lattal, 2005), or (e) response feedback (Dickson, Hayashi, Lightner, \& Lattal, 2009) was more resistant than higher-rate responding to (a) conditioned suppression (Blackman, 1968), (b) increasing schedule requirements (Lattal et al., 1998), (c) changes in the rate or distribution of SR (Metzger, 1992; Okouchi \& Lattal, 2006; Tatham et al., 1993; Urbain et al., 1978), (d) tests for response resurgence (da Silva et al., 2008) and (e) and the more common resistance-to-change disrupters (Dickson et al., 2009; Lattal, 1989; Nevin et al., 2001). There have been cases, however, in which lower-rate responding was not more resistant to change. Low-rate responding generated by (a) unsignaled delay of reinforcement (Bell 1999, Grace, Schwendemin, \& Nevin, 1998; Podlesnik et al., 2006; Podlesnik \& Shahan, 2008), or (b) response-independent food, (Nevin et al., 1990; Podlesnik \& Shahan, 2008; see also Experiment 3 in the present report) was not found to be more resistant to change. Finally, one study found that that low-rate responding generated by IRT contingencies was not more resistant to the more common resistance to change disruptors (Fath, Fields, Malott, \& Grossett., 1983; but see discussion of these procedures and results in Lattal, 1989). These differences in the resistance to change of low-rate responding generated by different schedules of reinforcement underscore the importance of the schedule that generates that response rate (Lattal, 1989; Doughty \& Lattal 2001).

As suggested by Lattal (1989) it is probably not the response rates themselves, but the contingencies that determine them that are responsible for the relation between response rates and resistance to change. Lattal suggested three potential strategies for distinguishing between differences in resistance to change resulting from response-rates per $s e$, and resulting from the contingencies that determine response rates. One such strategy is to compare effects on resistance to change of different contingencies that generate response-rate differences, while keeping reinforcement 
rate equal. Another is to compare effects of schedules of reinforcement that generate similar response rates with different contingencies of reinforcement (e.g., da Silva et al., 2008; Okouchi \& Lattal, 2006). The third strategy suggested by Lattal is to study resistance to change in behavior that occurs at different rates without having been exposed to an "explicit behavioral history designed to establish different response rates (1989, p. 201)." The three experiments in the current study result from an attempt to employ this third strategy, as they were designed to generate response-rate differences with contextual schedules of reinforcement, either on a separate response key (Experiments 1 and 2) or on a VT schedule (Experiment 3). The remainder of the current section entails an examination of data obtained using the first two of these strategies. Subsequent to that examination will be a discussion of the third strategy and findings from the experiments detailed the current study.

Low-Rate Responding Generated by DRL Schedules. Several authors have described comparisons of resistance to change or persistence of responding generated by different contingencies, but with the equal rates of reinforcement. Nevin et al., (1974, Experiment 5) found that when reinforcement rates were equated, responding under DRL was more resistant to disruption than was responding under DRH. Nevin tested preference for DRL vs. DRH schedules in the same pigeons and that the degree of preference for DRL terminal links was correlated within subjects with the degree to which DRL responding was resistant to change. The relation between resistance to change and preference in concurrent-chain schedules has been well supported and replicated (Grace \& Nevin 1997; Nevin \& Grace, 2000), and as a result, both are considered to be related measures of response strength (Nevin \& Grace, 2000). As discussed earlier, in an explicit test of response rate and resistance to change, Lattal (1989) replicated Nevin's (1974, Experiment 5) finding that low-rate responding was more resistant to change.

In a comparison of resistance to change of response sequences, Doughty \& Lattal (2001) found that responding was more resistant to disruption when pigeons were required to vary sequences of left- and right-key responses (vary condition), and less resistant to disruption when pigeons were required to respond in a fixed sequence of responses (repeat condition). This comparison was made using a multiple-chain schedule, and similar reinforcement rates were obtained across components. Response rate was lower in initial links that led to the vary condition than in initial links that led to the repeat condition. These authors argued that theories of response strength should take into account factors other than rate of reinforcement, and they proposed that response classes with more members are stronger than response classes with fewer members. As Doughty \& Lattal (2001) discussed, this interpretation can be applied to the difference in resistance to change of DRL-maintained responding: "perhaps under these low-rate contingencies, responses other than the one necessary for reinforcement are strengthened, whereas only the response necessary for reinforcement is strengthened under the high-rate contingencies. In other words, under each of these contingencies the nominal or descriptive response class is similar in number but the functional response class is not (p. 213)." In the case of the present set of experiments it is possible that, in contrast to experiments in which DRL schedules were used to generate low response rates, the functional response classes generated by the contingencies were similar across components; as a result lower-rate keypecking was not more resistant to change that was higherrate keypecking.

In another study that showed greater resistance to change of DRL responding, electric shock was used as a disruptor (Blackman, 1968). Low and high response rates were generated by a multiple DRL/FI-limited-hold (LH) schedule, and similar reinforcement rates were obtained across components. At lower shock intensities, when a tone that terminated in shock was added as a disruptor, response rates in the DRL component increased. As shock intensity increased, response rate gradually decreased. In comparison, barpressing in the FI-LH component was suppressed at lower shock intensities. Blackman suggested that the increase in response rate at low shock intensities resulted from adventitious punishment of responding (other than barpressing) that had been generated by the DRL contingency. With low response rates, the onset of the tone was most likely to follow behavior other than barpressing. Blackman postulated that the punishment of this other responding was responsible for the increase in response rates, which translated to less disruption of the barpress response. Doughty and Lattal's (2001) argument that response classes with more members have greater response strength does not contradict this interpretation. Both pausing and the nominal operant response (in this case, barpressing) are required for reinforcement on the DRL schedule, whereas only barpressing is required for reinforcement in the FI-LH schedules. The functional response class in the DRL component, therefore, has more members than the functional response class in the FI-LH component, and as a result DRL responding should be more resistant to change.

Greater resistance to change of low-rate behavior generated by DRL also has been found with disrupters that potentially increase response rates both in tests of persistence in the face of changed reinforcement contingencies and tests of resistance to resurgence after a condition that reduces responding. When behavior previously maintained by DRL and FR (or DRH) schedules with equal mean IRIs is reinforced on FI schedules, the former DRL 
responding changes more slowly (Freeman \& Lattal, 1992; and Metzger, 1992; Urbain et al, 1978). Tatham et al. (1993) extended the generality of the finding that lower-rate DRL responding is more resistant to change by comparing persistence under FI-30-s schedules of responding generated by three different DRL-schedule histories. These authors found that DRL-60-s responding was more persistent in rats than DRL-30-s or DRL-10-s responding. In these studies of behavioral history resistance to change was greatest when response rate was lowest, and when DRL schedules required the longest pauses. Consistent with this generalization were the results of da Silva et al. (2008, Experiment 2), which established high and low rate responding in pigeons in a concurrent tandem VIDRL/tandem VI-FR, with similar rates of reinforcement across response alternatives. Next, in a responseelimination condition food was delivered on a differential-reinforcement-of-other-behavior (DRO) schedule. Finally, responding on each of the two keys was measured under EXT. In this last condition behavior generated with a DRL requirement resurged less than did behavior maintained by similar rates of reinforcement, but with an FR requirement. It is possible that less resurgence was measured on the former DRL key because both pausing and responding were reinforced under the temporally remote DRL condition, but it is also possible that response allocation was influenced by a carryover effect of switching between keys engendered in the first condition of the experiment. In their Experiment 3 da Silva and her colleagues adjusted tandem schedules (VI DRL and VI DRH) to generate similar response rates with different rates of reinforcement and found little difference in resurgence between the two alternatives. These authors concluded that the relation was stronger between response-rate differences and resurgence than between reinforcer-rate differences and resurgence. It is possible that in Experiment 3 the two functional responses were more similar between alternatives than in Experiment 2. Again, it may be the size of the functional response unit or class (and not the response rate, per se) that is a critical factor in determining subsequent behavior; which, in this case, is measured during a test for resurgence. Additional research is necessary to determine the mechanism responsible for this difference in relative resurgence across experiments.

Low-Rate Responding Generated by Interval Schedules. In addition to DRL contingencies, interval schedules have been shown to generate an inverse relation between response rate and resistance to change. Compared to ratio schedules, yoked interval schedules tend to result in lower rates of responding. Nevin et al. (2001) showed that in addition to generating lower rates of responding than ratio schedules, responding on yoked VI schedules was more resistant to disruption by prefeeding, free food during ICIs, and EXT. Additionally, in a concurrent-chains choice procedure, initial links of concurrent-chain schedules that were followed by yoked VIs were preferred over VRs. This finding of greater resistance to change of yoked VI than VR responding was supported by Lattal et al. (1998) who found that pigeons would continue pecking longer in yoked VI components than in VR components as reinforcement schedules were progressively leaned. Although it should be noted that Lattal and his colleagues did not obtain consistent differences in response rate, this fact only adds to the strength of the argument that it is not response-rate differences per se that are responsible for greater resistance to change of low-rate responding, but the schedules that generate these differences. It is not clear how well these comparisons of interval- and ratio-maintained responding are described by Doughty and Lattal's (2001) finding that response classes with more members are more resistant to change. In the ratio and interval schedules used in these studies, reinforcement is always delivered immediately following a single response. It is possible that greater variability in IRTs under the interval schedule reflects a larger response class, but this supposition is speculative. In discussing the results of Lattal et al. (1998) and Nevin et al. (2001), Shull, Grimes, and Bennett (2004) emphasized the importance of considering the functional response unit. Response-rate differences are not the same when bouts of responding are counted, as opposed to single responses. Shull and colleagues argue that the greater post reinforcement pausing engendered by ratio schedules may indicate avoidance of bout initiation. It is possible that an aversive element of ratio schedules is responsible for less preference for and less resistance to change in the ratio schedules. Again, additional research on factors related to resistance to change is needed. The important point in relation to the current set of experiments is that the structure of the response unit generated by a particular schedule of reinforcement may be a critical determinant of responding in a test for behavioral persistence.

Low-Rate Responding Generated by Additional Stimuli. Another manipulation that has resulted in both low-rate responding and greater resistance to change is the presentation of stimuli in one context and not another. Lower-rate responding, for example, occurs in contexts in which reinforcement is delivered simultaneous with or immediately subsequent to brief auditory or visual signal, and this responding is also more resistant to change than responding in an equivalent condition with no signal, or with uncorrelated signals (Reed \& Doughty, 2005; Roberts, Tarpy, \& Lea, 1984). A similar effect has been found in a study of response feedback across two components of a multiple-FI-60-s/FI-60-s schedule. In one component each response was followed by a brief flash of light (response feedback) and in the other component there were no added stimuli (Dickson et al., 2009). The three pigeons in this experiment responded at a lower rate in the component with feedback. Responding in the feedback component was 
also more resistant to prefeeding. Reed and Doughty (2005) extended Doughty and Lattal's (2001) interpretation of response strength by assuming that the signal in their study led to responses other than the one that was recorded. They postulated that the presence of these unmeasured responses resulted in a larger functional response class in the component with the signal, and this larger response class was more resistant to change.

Low-rate Responding Generated by Unsignaled Delay to Reinforcement. Studies that have used unsignaled delayed reinforcement to reduce response rates have not found that the resulting lower-rate responding is more resistant to change than responding maintained by immediate reinforcement with approximately equal IRIs (Bell, 1999; Grace et al., 1998; Podlesnik et al., 2006; Podlesnik \& Shahan, 2008). This result seems to challenge the argument that operant classes with more members are more resistant to change, because it has been shown that unsignaled delay increases the prevalence of other responding, for example, hopper-observing behavior (Schaal, Shahan, Kovera, \& Reilly, 1998). If increasing "other" behavior increases the size of the operant class and resistance to change when signals are added (as in Dickson et al., 2009; Reed \& Doughty, 2005; Roberts et al., 1984) why doesn't an increase in "other" behavior result in greater resistance to change when there is an unsignaled delay to reinforcement? Either these other responses are not members of the functional response class, or resistance to change can be seen only in the response that is more reliably contiguous with reinforcement. It is possible that increased resistance to change would be seen if the other response, the one that more frequently immediately precedes reinforcement, were used to measure response strength. Several studies have shown that responding that is nearest reinforcement in chained schedules is the most resistant to change (Doughty \& Lattal, 2001, in the "vary" component; Nevin, Mandell, \& Yarensky, 1981; Williams, Ploog, \& Bell, 1995). Perhaps it is not only the number of members of a functional response class, but also the contiguity of the particular response with reinforcement that affects the resistance to change of a particular response within a response class. These differences in the resistance to change of low-rate responding generated by different schedules of reinforcement underscore the importance of focusing on the role of the schedule that generates rate (Lattal, 1989; Doughty \& Lattal 2001), and the specific effects of that schedule on the organization of responding (Schaal et al., 1998). Perhaps response rate is irrelevant to resistance to change, as it appears to have been in the current study.

Resistance to Change of Behavior with Equal Response Rates and High and Low Reinforcement Rates. The second strategy suggested by Lattal (1989) for distinguishing between effects of response rates and contingencies on resistance to change was to compare effects of schedules of reinforcement that generate similar response rates with different contingencies of reinforcement (e.g., da Silva et al., 2008, Experiment 3; Okouchi \& Lattal, 2006). Okouchi and Lattal (2006) used this strategy in their study of reinforcement history effects. They built a history of similar response rates with different rates of reinforcement using multiple-tandem-VI-DRL schedules, and tested history effects using FI schedules with IRIs that were equal to either the richer or leaner historical schedule. Unlike previous studies of behavioral history effects, in this study behavioral resistance to the schedule change was measured in terms of divergence of response rates, rather than convergence of response rates. In general, responding with a history of relatively rich rates of reinforcement changed more rapidly than did responding with a history of relatively lean rates of reinforcement. In comparison to history studies which compare persistence of high vs. low response rates (e.g., Freeman \& Lattal, 1992), history effects in Okouchi and Lattal (2006) are small. These authors concluded that historical response rates contribute more to history effects than do historical reinforcement rates. A similar conclusion was drawn by da Silva et al. (2008) which compared resurgence of responding after histories of different response rates, with similar reinforcement rates (Experiment 2) or different reinforcement rates, with similar response rates (Experiment 3). Responding was established with a concurrent-tandem-VI-30-s-DRL / tandem-VI-30-s-DRH schedule, and reinforcement rates differences were programmed using a Stubbs-Pliskoff (1969) procedure whereby the same VI schedule was operative in for each response key, but reinforcement was set up on only one response key. For example, the key with the DRL component may be scheduled to deliver reinforcement two times as often as the key with the DRH component. After responding was judged to be stable, it was eliminated with a DRO schedule. Finally, EXT was effected and responding resurged. Relative differences in resurgence were not related to relative reinforcement rates. As noted above, however, greater resurgence had been related to greater response rate in Experiment 2 of the same study. In summary, experiments that established similar response rates and different reinforcement rates across two conditions have resulted in less differential responding under subsequent tests for effects of this history than have previous experiments that established response-rate differences. It is possible that these studies that generated similar response rates also generated similar-sized functional response units. Evidence of this possibility has not been borne in the literature to date, and the use of both DRL and DRH contingencies in da Silva et al. (2008) may have been less likely to result in similar functional units, as these differential contingencies on response rate are similar to those that generated response-rate differences in many previous studies of behavioral history. 
Greater history effects consistently have occurred in tests that followed a period of response-rate differences using different contingencies on responding, including rate-controlling schedules of reinforcement, variability requirements, interval schedules, added stimuli, and unsignaled delay to reinforcement, than in tests that followed a period of reinforcement-rate differences and similar response rates. Although there is evidence that the schedules that generate these response-rate differences determine these history effects, it remains unclear what features of these schedules or what features of the responses they generate, are most strongly related to the endurance of effects of historical conditions of responding.

The third strategy suggested by Lattal (1989) for disentangling the relative contributions of reinforcement and response rate history to response rate in subsequent conditions was to manipulate response rates without directly manipulating reinforcement rates. The following discussion of experiments that relate to this third strategy will address the experiments from the current paper and other studies in which response rate was manipulated using of additional sources of reinforcement.

\section{The Use of Context to Generate Response-Rate Differences}

A few studies of behavioral persistence have attempted to separate response rates using putatively identical contingencies. These contingencies are putatively identical because in each case, some environmental event has been arranged in attempt to manipulate response rates. As Lattal (1989) noted, if response rates are different it could be assumed that these differences result from functionally different contingencies. Under such a functional definition of "contingency," the experiments described in this report could be considered to fit into Lattal's first category of solutions to the problem for determining the role of historical response rate on resistance to change; because response rate was different across components, and reinforcement rate was the same for the target response across components. No matter what definitions are used for contingency or context, the current experiments provide data that bear on the problem of delineating the relative roles of behavioral and reinforcement history in determining response strength by affecting response rates with contextual schedules of reinforcement in a history-building condition. These contextual schedules of reinforcement were arranged on an alternative response key in Experiments 1 and 2, and delivered independent of responding in Experiment 3. Reinforcement for the target response was always immediate, and was delivered on the same schedules in Experiments 1 and 2, and with equal interfood intervals across components in Experiment 3. These manipulations should not have constrained the target response rate as directly as manipulations of reinforcement contingencies programmed for the target response would have. The discussion of the relative contributions of response and reinforcement rate continues below with consideration of the three experiments entailed in the current study, and a few related experiments.

Accounting for differential persistence in Experiments 1 and 2. In Experiments 1 and 2 there was a failure to generate consistently large response-rate differences in the history-building condition. Small response-rate differences, however, did occur for each pigeon. For each pigeon in Experiments 1 and 2, response rate on the target response key (the side key) was greater in Component B, the component for which the reinforcement rate for the target response was rich, relative to the reinforcement rate for the alternative response (the center key). In general, resistance to change was greater in Component B; this finding is inconsistent with studies that have found historically lower-rate responding to be more persistent. Additionally, greater persistence in Component B is inconsistent with predictions from the behavioral momentum literature. If all of the reinforcers delivered in each component are considered, responding in Component A should be more resistant to change.

How can we account for the greater persistence in Component B, the component with a relatively lean reinforcement schedule on the center key? One account is offered by the assumptions made by Belke (1992). That is to say, response strength on the side key in Component B should be greater than on the side key in Component A, because in Component B the side key was relatively rich within its component, and in Component A, the side key was relatively lean within its component. This assumption that the side key in Component B acquired greater value as a function of its historic status as the relatively rich response option is challenged, however, not only by the fact that this argument is one that ascribes hypothetical "value" to the response key stimuli, but also by the possibility that response rate in preference tests similar to those used by Belke was affected by the pigeons' historic patterns of moving toward and away from the side keys (Gibbon, 1995; Grace \& Savastano, 1997; McDevitt \& Bell, 2008; Nevin \& Grace, 2000; Zentall, Weaver, \& Sherburne, 1996; Williams \& Bell, 1996, Experiment 2).

A second account for the greater persistence on the Component-B side key in Experiments 1 and 2 is related to Staddon's discussion of interim responding (Hinson \& Staddon, 1978; Staddon \& Simmelhag, 1971), and Harper and McLean's (1992) discussion of behavioral reallocation. Following this account, when the center key (the interim response) was no longer available in history testing, responding that would have occurred on this key in history 
building was reallocated in each component to the side key. More responding occurred on the center key in Component A than B, and therefore, when this responding was no longer available, response rate on the side key in history testing increased more in Component A than B.

A third account for the greater persistence on the Component-B side key is related to Doughty and Lattal's (2001) finding that more complex response classes are more resistant to change, and Tatham et al.'s (1993) finding that responding maintained by DRLs with longer IRT requirements are more persistent than responding maintained by DRLs with shorter IRTs. It is possible that the concurrent schedules in history building resulted in a larger response unit or a more complex response class with respect to the Component B side key, as compared to the Component A side key. In history building, pigeons allocated more pecking to the side key than the center key in Component B, and more pecking to the center key than the side key in Component $\mathrm{A}$. If longer stretches of time were spent on the center key in Component A than Component B, the obtained delay to reinforcement after switching to the side key in Component A would have been shorter than in Component B. That is, reinforcement on the side key in Component A would have been more likely to have been set up sooner after the elapse of the COD requirement than in Component B. Less complex or smaller response units may have developed for the side key in Component A, as a result of a relatively brief delay to reinforcement, and these smaller response units were less persistent. This suggestion is speculative, but further research could contribute fruitfully to the specification of contextual features (i.e., alternative sources of reinforcement) that lead to topographically different functional response units and the relation between the structure of these units and resistance to change.

A fourth account for greater persistence in Component B is related to the common finding that behavioral resistance to change depends in part upon the similarity of stimulus conditions in the baseline and testing conditions (Freeman \& Lattal, 1992; Harper, 2001; McIlvane \& Dube, 2001; Metzger, 1992; Nevin \& Grace, 2001; Okouchi, 2003; Okouchi \& Lattal, 2006). According to Harper (2001), in the vernacular of the behavioral momentum literature, if stimulus conditions are changed in functionally different ways across components between baseline and testing conditions, then unequal disruptive forces have been applied across components. The use of unequal disruptive forces limits the interpretation of behavioral resistance to change. Even if a stimulus change appears to be comparable from the perspective of the experimenter, for example, a change from illuminated keys to one illuminate key, such a change may be functionally different with respect to the degree to which it impacts behavior across components. In the case of Experiments 1 and 2, the stimulus conditions were changed when the history testing condition began. This change may have been functionally greater in Component $\mathrm{A}$ than $\mathrm{B}$, because the pigeons allocated more responding to the center key in Component $\mathrm{A}$, and the center key was not illuminated nor available in history testing. If the topography of stimulus control were different across components, for example, if the center key had been a more important component of the controlling stimulus class in Component A than B, then the elimination of the center key stimulus may have been more disruptive in Component A than B. Another stimulus change that was different between Components $\mathrm{A}$ and $\mathrm{B}$ was the overall schedule of reinforcement. The greater change in overall rate of reinforcement in Component A could have decreased the likelihood of stimulus generalization of historical responding in that component.

Each of the four accounts expounded above provide an explanation for the greater change in response rate on the side key in Component A in the testing conditions of Experiments 1 and 2. Only two of the four accounts, however, can account for the direction of this change, that is, for the greater increase in response rate in Component $\mathrm{A}$. The first and fourth accounts do not explain why response rate would increase more in Component A than B.

Reinforcement rate decreases in the test condition, and there is no explanation on the basis of the historic relative value of the side keys or the relative similarity of the stimulus conditions in history building and history testing that justify the increase in response rate in history testing. The third account could explain the greater increase in response rate in Component A using a response-organization account. For example, response rate would increase in Component $\mathrm{A}$ if the response rate during bouts of responding were greater on side-key $\mathrm{A}$ than $\mathrm{B}$, and the history testing condition resulted in longer bouts. The second account seems to be the best account for the differential increase in response rate seen in Components A and B. The account based on reallocation of responding predicts an increase in response rate on the side key if the other alternative is no longer available.

Accounting for Differential Persistence in Experiment 3.Like Experiments 1 and 2, Experiment 3 used context to generate response-rate differences. Experiment 3 did this by delivering a portion of the reinforcers on a VT-30-s rather than a VI-30-s schedule in Component A. Response-independent food deliveries accounted for 75\% to $100 \%$ of all food deliveries in Component A, whereas reinforcers were delivered on a VI-30-s schedule in Component B. The response-independent food resulted in a lower response rate in Component A. When both components were changed so that reinforcement was delivered on an FI-30-s schedule, response rate tended to 
increase more in Component $\mathrm{A}$ than $\mathrm{B}$, and therefore responding in Component $\mathrm{B}$ was considered to be more persistent. As in Experiments 1 and 2, this finding was inconsistent with the common finding that lower-rate responding is more resistant to change (e.g., Lattal, 1989; Tatham et al., 1993). It is also inconsistent with the behavioral momentum literature, which would predict no difference in resistance to change across components with equal rates of food delivery across components (Nevin \& Grace, 2001; Nevin et al., 1990).

How well do the four accounts for the greater persistence in Component B in Experiments 1 and 2 apply to the same result in Experiment 3? The account related to Belke (1992) could apply if we consider the VT food to be a contextual schedule of reinforcement. The rate of reinforcement for pecking the key was higher in Component B, and therefore the response key in Component B should be of greater value than in Component A. It follows that more pecking should occur on the key in Component B. This explanation, however, does not justify the direction of the change, an increase in response rate in Component $\mathrm{A}$, and a decrease in response rate in Component $\mathrm{B}$.

The reallocation account also works for Experiment 3. Responding that occurred immediately prior to VT food deliveries could have increased in the history-building condition. In history testing, these responses would have been extinguished, because all reinforcers in history testing were contingent on keypecking. According to this account the alternative or interim behavior that would have been reinforced in the history-building condition would have been reallocated to the reinforced topography, keypecking. Because there was no alternative reinforcement in the historybuilding condition in Component $\mathrm{B}$, there was less change in response rate in the history-testing condition. This account explains both the greater change in response rate in Component $\mathrm{A}$, and the direction of the change: an increase in response rate. The response reallocation account is, however, more difficult to apply in Experiment 3 than in Experiments 1 and 2, because the supposed alternative responding is unmeasured. There is no direct evidence that any other response has increased as a function of response-independent food delivery. A suggestion for a procedure that would allow for the measurement of interim responding is offered by Lattal and Boyer (1980). These authors defined "pausing" as a period of $5 \mathrm{~s}$ with no keypecks, and found that they could manipulate the allocation of responding between pecking and pausing by shifting contingencies under a concurrent FI/Tandem-VTDRO schedule, in a manner similar to other concurrent schedules with two response alternatives. The resulting ratio of time spent pecking to time spent pausing fit nicely with the matching law (Herrnstein, 1970). One suggestion for clarifying the role of competition from interim responding is to use a history-building condition in which pausing can be explicitly reinforced and measured in one component. The use of such a procedure could lead to stronger support for a reallocation account of the increase in response rate in Component A. Using a concurrent VI/TandemVT-DRO schedule in one component would allow for the measurement of both types of responses, and an analysis of potential reallocation of responding from pausing to pecking.

The application of a response-organization account to the problem of greater persistence in Component B is tricky. It might be expected that responding in Component A would have been more variable than in Component B; adventitious reinforcement could have lead to the development of a wider variety of responses that, initially by chance, preceded response-independent food and thus were strengthened (cf. Skinner, 1948, but Skinner used FT). The resultant greater variability in response topography could be expected to be associated with greater resistance to change, provided they could be considered to be part of the same operant response class (Doughty \& Lattal, 2001). Keypecking in Component A, however, was not more persistent than keypecking in Component B. This finding is in concert with experiments that have used response-independent food or delay to reinforcement in one component of a multiple schedule.

Although Nevin et al. (1990) found that adding VT food resulted in greater resistance to change, this effect was only present when there was a greater rate of food delivery in the VT component. they did not conclude that VT food affected resistance to change when interfood intervals were equal across VI+VT and VI components of a multiple schedule (Conditions 5, 20\% VT and 6, 80\% VT). Response rate was, however, less resistant to change in the VIVT component in two of the three pigeons (Figure 3., Nevin et al., 1990). The current Experiment 3 and Podlesnik and Shahan (2008; Experiment 2, 80\% VT) found differential resistance to change between components with equal rates of food delivery when a proportion of these were delivered independent of responding. In both Podlesnik and Shahan (2008; Experiment 2), and Experiment 3 from the current study the component with response-independent food was less resistant to change; and in most other studies where low-rate behavior is more resistant to change, the response-reinforcer dependency always is present in both.

It is difficult to interpret the results of Experiment 3 through the lens of response organization, because there is no measure of the functional response unit in the component with VT food, nor is there any clear empirical precedent on which to build an hypothesis regarding its size or complexity. Responding under VT schedules is necessarily uncontrolled by the experimenter, and there is a wide range of behavior that could result, as can be seen in the range 
of response rates in history building across pigeons in Experiment 3. The resistance-to-change results of each Experiment 3, Podlesnik \& Shahan (2008, Experiment 2, response-independent condition), and probably also of Nevin et al. (1990, Experiment 1, Conditions 5 and 6 in) may best be accounted for by a consideration of responsereinforcement contiguity. Responding in components with response-independent food is affected in resistance to change tests similarly to responding in components with unsignaled delay to reinforcement. Each of these manipulations results in behavior that is less resistant to change, as compared to responding maintained by reinforcement that immediately and contingently follows responding (Podelesnik \& Shahan, 2008, Experiment 2). It is important to note, however, that this diminished resistance to change of behavior maintained by delayed or response-independent reinforcers is seen only in studies in which the rate of reinforcement is equal across components.

As discussed previously, in studies of resistance to change of behavior maintained by unsignaled, delayed reinforcement, authors have noted that one plausible explanation for the diminished resistance to change is a change in the structure of the response, or the increase in a potentially competing response, namely, the response that occurs closer in time to the reinforcer (Grace et al., 1998). The size of the functional response unit under unsignaled, delayed reinforcement is larger; a peck followed by a pause is required for reinforcement, rather than a single peck, as in a simple VI schedule. Doughty and Lattal (2001) showed that larger or more complex functional units are more resistant to change, but also found that responding that occurs closer in time to the reinforcer is more resistant to change. In this case, pausing, or any behavior a pigeon tends to engage in between the last peck before a reinforcer and start of reinforcement, should be more resistant to change than pecking.

Following the response-organization account, in resistance tests that function to decrease response rate, behavior maintained by delayed reinforcement (e.g., pecking) should decrease more rapidly than behavior maintained by immediate, contingent reinforcement (e.g., pausing). This quicker decrease potentially could be attributed to three factors: the greater resistance to change of pausing, the lesser resistance to change of pecking, and the greater resistance to change of this pattern of peck-then-pause-responding. A similar response-organization-based explanation can be applied to the diminished resistance to change of behavior maintained in the context of responseindependent reinforcer delivery. Pecking does not always precede food in such a context, but some other response might, and as a result, all of these responses are likely to occur in testing conditions. If the functional unit of responding is larger as a result of the response-independent food delivery, the entire response unit should be more resistant to change. It does not necessarily follow, however, that the measured response, pecking in this case, should be more resistant to change than the response that occurs when the pigeon is not pecking.

To what extent is the greater resistance to change of this "other" behavior responsible for the quicker or greater change in response rate? One way to investigate this problem might be to repeat the baseline conditions of resistance to change studies that have compared responding under conditions of delayed or response-independent reinforcement with responding under a simple, immediate, contingent schedule, such as VI. Next, instead of testing for resistance to change using the usual disruptors that typically decrease response rate, a different schedule of reinforcement may be implemented as a test condition. If the "other" behavior is more resistant to change, this could be expected to retard the increase in rate of pecking. If the other behavior is not more resistant to change as a function of having been more contiguous with responding, then this could be expected to promote the increase in rate of pecking. Of course this method would bring a new potential problem to these tests - that is, the changed-to schedule would have to be selected carefully. The introduction of a different schedule of reinforcement could result in rapid changes in responding, and these rapid changes could prevent differences from being discerned between the pre-conditions.

In summary, regarding the response-organization account of resistance to change, the way in which VT food affects the functional response unit is an open question. Effects of VT reinforcement vary across pigeons and across time, as there is no predetermined relation between responding and food delivery times in VT. Taken together with the resistance to change data from experiments with delayed reinforcement, it seems that the contiguity between a response and a reinforcer is an important determinant of the resistance to change of that response. This conclusion is inconsistent with the stimulus - reinforcer relation account of resistance to change forwarded by Nevin and some of his colleagues (Nevin et al., 1990; Nevin \& Grace, 2000). The stimulus-reinforcer relation account has been challenged by experiments that have used equal rates of reinforcement across components or groups and have found lower-rate responding (generated in a variety of procedures) to be more resistant to change. The stimulusreinforcer account of response strength has also been challenged by experiments that have found responding that is maintained by delayed reinforcement or in the context of response-independent reinforcement to be less resistant to change. Nevin and his colleagues later acknowledged that other factors play a role in resistance to change, but that 
the role of overall reinforcement rate in a context is the most strongly related variable in determining resistance to change (e.g., Nevin et al., 2001). The dominant role of reinforcement rate as the determinant of resistance to change is well supported in the resistance to change literature (e.g., Nevin et al., 1990; Podlesnik \& Shahan, 2008). The degree to which resistance to change depends upon the immediacy of reinforcement could be clarified in further research, by testing resistance to change of behavior maintained on multiple-mixed or -concomitant VI-VT/VI schedules with different proportions of VT food, and equal interfood intervals across components. The available evidence supports the conclusion that the structure of the functional operant and the contiguity between responding and reinforcement do affect behavioral persistence, but the influence of these variables can be overwhelmed by effects of differences in reinforcement rate. It is a general finding that lower-rate responding more resistant to change when the reinforcement rate is equal, and reinforcement is immediate and contingent on the target response.

The final account for greater persistence in Component B in Experiments 1 and 2 is the stimulus similarity account. This model applies well to Experiment 3. There was no change in the stimulus lights in the chamber in either component between the history-building and the history-testing conditions, and there was no change in the mean interfood interval. There was, however, in both components, a change in the temporal distribution of reinforcers. Perhaps most importantly, there was a large change in the degree of contingency between keypecking and reinforcement in Component A, and there was no change in the degree of contingency in Component $\mathrm{B}$. According to a stimulus similarity account, this greater change in contingency in Component A would predict greater changes in response rate in Component $\mathrm{A}$, and quicker adaptation to the novel schedule in the history testing condition.

Taken together, evidence from studies that have fit Lattal's (1989) three suggested methods of delineating relative contributions of behavioral and reinforcement history supports the argument that the schedules that generate response-rate differences, rather than the rate of responding per se, are responsible when low-rate responding is found to be more resistant to change. The present study contributes to this body of evidence by using contextual schedules of reinforcement to manipulate response rates in two components of a multiple schedule in a historybuilding condition. This use of contextual schedules provided a novel test of the persistence of low-rate responding in the face of changed contingencies. In previous studies of behavioral history, rate-controlling schedules such as DRL have been used to generate response-rate differences. Evidence from the experiments discussed above indicates that response rate and behavioral persistence are affected through a change in the structure or organization of the response unit. Additional research should continue to address effects of a variety of historical reinforcement schedules, contexts, contingencies on response rate, degrees of dependency, and degrees of immediacy on persistence in the face of changed conditions. Further investigation of the degree of change in response rate and response organization across a range of disruptors, schedule changes, and context changes would contribute to and more complete understanding of response strength and better specification of factors that lead to behavioral persistence.

\section{Analysis and Interpretation of History Effects}

In the current study, the use of context to manipulate response rates in two components of a multiple schedule in a history-building condition provided a novel test of the persistence of low-rate responding in the face of changed contingencies. In previous studies behavioral history, rate-controlling schedules have been used to generate response-rate differences. Typically, in the history-testing conditions that followed, response rates in each component converged gradually as responding came into contact with the new contingencies. In these studies, once response rates converged, they continued to occur at similar and relatively stable asymptotic rates. In the current set of experiments, and especially in Experiments 1 and 2, the use of contextual schedules of reinforcement to generate response-rate differences resulted in behavior that was highly variable in the history-testing condition. Additionally, response rates between components overlapped relatively early in the testing condition. The resulting sign changes and the difference between response rates in the two components did not appear to reflect convergence as it has been characterized in previous studies of behavioral history. The sign of the difference in response rates changed several times in most pigeons prior to the behavior in each component reaching asymptote. Responding in the historytesting condition, therefore, appeared to continue to be affected by the historical condition after the response rates changed direction. This variability in responding in the history-testing condition indicated that responding was affected by inconsistent sources of control over the course of that condition.

Although in Experiments 1 and 2 there was no relation between response-rate differences between the Component A and B side keys in history building and the number of days response rate in B was greater than response rate in A in history testing, in Experiment 3 this relation was apparent. In Figure 30, a scatterplot for each of the three experiments shows the proportion of sessions in which response rate was greater in Component $\mathrm{B}$ (the component that had been designed to engender higher response rates) than A (the component that had been designed to 
engender lower response rates) as a function of the proportional response rate $(\mathrm{B} / \mathrm{B}+\mathrm{A})$ on the side keys in history building. In Experiment 3 there is a stronger relation between response-rate differences in history building and proportion of days with greater response rate in Component B in history testing. It appears that the availability of alternative reinforcement for pecking the center key affected subsequent responding differently than did the presence of VT food in Experiment 3, or the rate-controlling contingencies on single keys in previous studies of behavioral history. Because changes in response rate were not as straightforward as in previous studies of behavioral history, additional measures of persistence were used. In addition to rate of change over short and long ranges of sessions and temporal distribution of responding, the current study included measures of variability and rate of change of variability. Response rates were used to calculate the number of sessions in history testing for which response rate was higher in Component $\mathrm{B}$ than $\mathrm{A}$, the number of sessions to the first two sign changes, the number of sessions to convergence, the number of sessions to asymptote, and the number of sessions to stability. The asymptotic level for each component was experimentally defined as the mean of the last 10 sessions. In many pigeons, by the end of the history-testing session responding was still variable enough that the criteria for the " $10 \%$ Terminal" measure were never met. That is, for some pigeons, responding was never within $10 \%$ of the asymptotic level for at least five consecutive days. The use of a variety of measures of change in responding yields a more complete picture of how responding changed as it contacted the new contingencies.

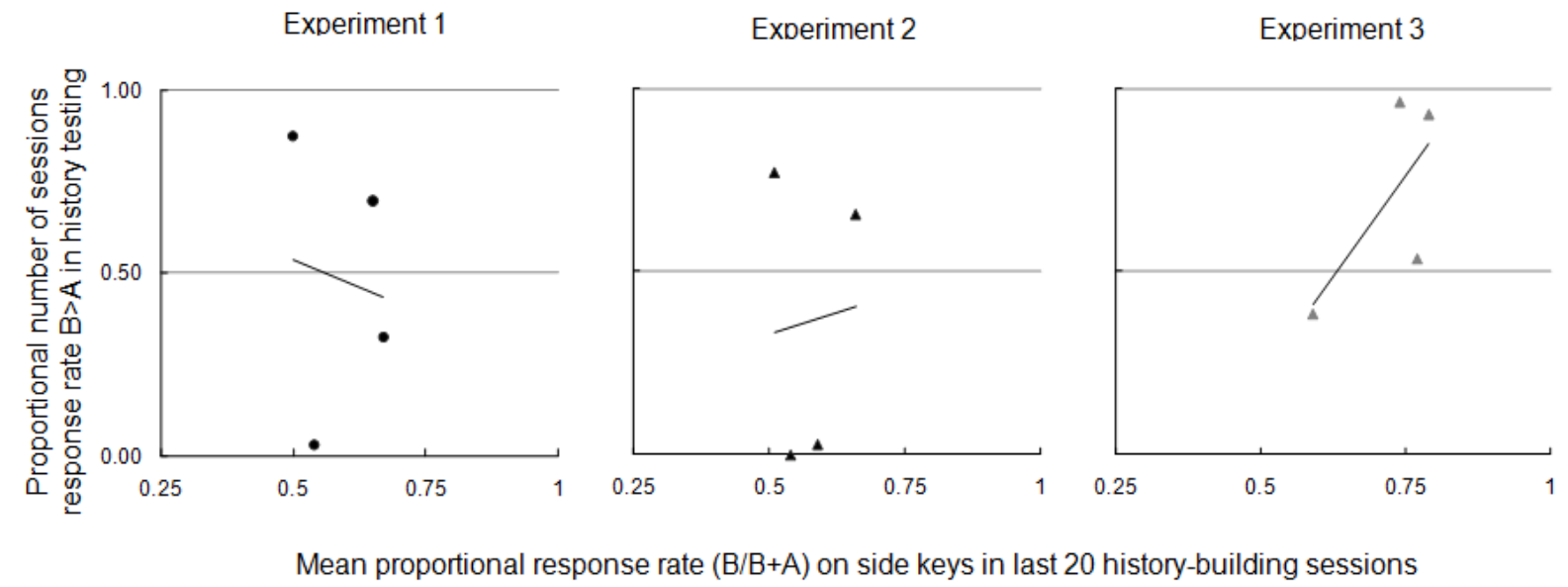

Figure 30. A scatterplot for each of the three experiments shows the proportion of sessions in which response rate was greater in Component $\mathrm{B}$ than $\mathrm{A}$ as a function of the proportional response rate $(\mathrm{B} / \mathrm{B}+\mathrm{A})$ on the side keys in history building.

There has been evidence for multiple or conflicting mechanisms of control in studies of resistance to change. In some cases behavior has changed directions during the test, for example by increasing before decreasing (e.g., Blackman, 1968; Lattal, 1989; Nevin et al., 2001; McLean \& Blampied, 2005). This kind of result indicates more complex control of responding than simply the difference between a high and low rate of reinforcement. For example, Blackman (1968) found that responding that was previously reinforced on a DRL schedule increased in the presence of a shock-correlated tone. He hypothesized that pausing was punished by this tone, and that this resulted in an increase in barpressing, even under conditions of punishment. As the shock intensity was increased, the rate of barpressing decreased. It is important in these cases in which an experimental manipulation may result in the increase or decrease of response rate to use the absolute difference from baseline when calculating a difference score (see also Grace \& Nevin, 1997; Nevin et al., 2001).

Figure 31 shows for each pigeon in each experiment a measure of persistence in the first 10 sessions of history testing: the log ratio of response rate in history testing and the mean response rate in the last 6 sessions of history building. Because the manipulation used in the test did not result in response rate changes in the same direction, the absolute differences from the history-building response rate were used to calculate difference scores for each pigeon. Figure 32 displays the absolute difference scores for each pigeon as a function of the log ratio of historical response rates $[\log (\mathrm{Pas} / \mathrm{Pbs})]$. The absolute difference score was determined first by calculating the within-component $\log$ ratio of history building response rate for each session of history testing. The absolute value was taken for each of 
these ratios, and the result was summed within components. Finally, the sum from Component B was subtracted from the sum from Component A. A positive result indicates more change in Component A, and thus greater resistance to change in component $\mathrm{B}$. The relation between absolute difference scores and response-rate differences between components in the history-building condition relation is shown for the first 5 sessions for each pigeon, which is common in resistance to change studies (open symbols), and for the first 70 sessions (67 for pigeon 975; closed symbols). The higher the value on the ordinate axis, the greater the resistance to change of Component B, relative to Component A. Differences between relative resistance in the first few sessions of history testing and later sessions indicate inconsistent mechanisms of control. Although it may be difficult to delineate to what degree a single manipulation may be affecting response rate when factors that may increase or decrease response rate are simultaneously effected, it is important to consider measures that may reflect these influences. Also, as McLean and Blampied (1995) have noted, the rate of change in response rate between two components may be different from the extent of change. Further investigation of historic variables that control the extent of change in response rate would be interesting, especially if these variables differ from those that control rate of change.

Experiment 1
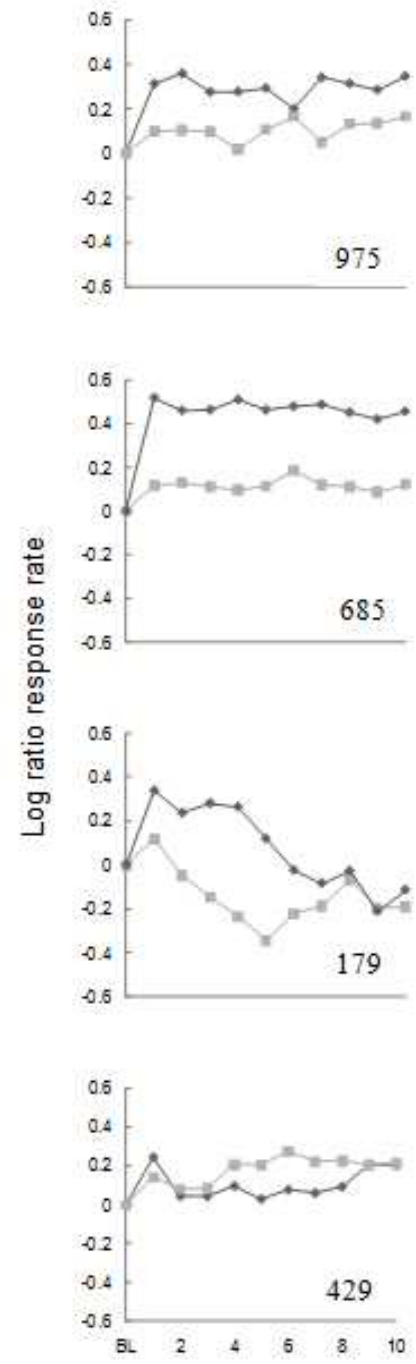

Experiment 2
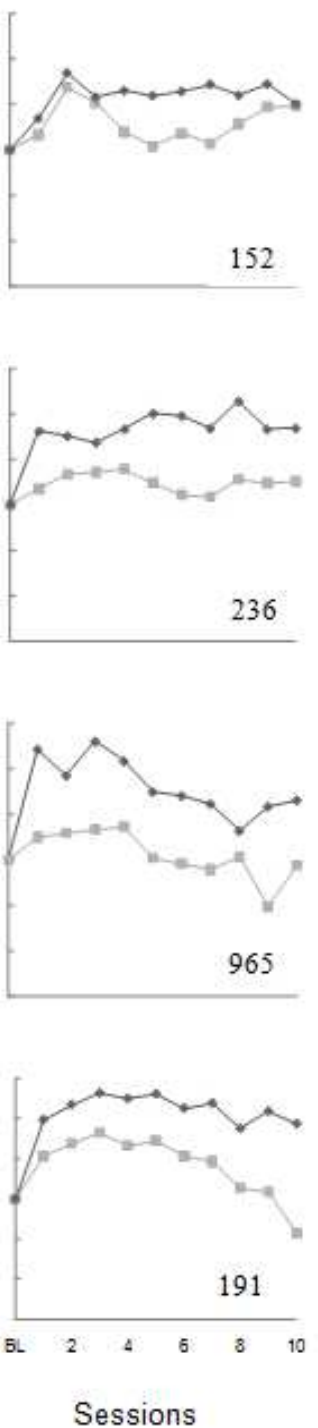

Experiment 3
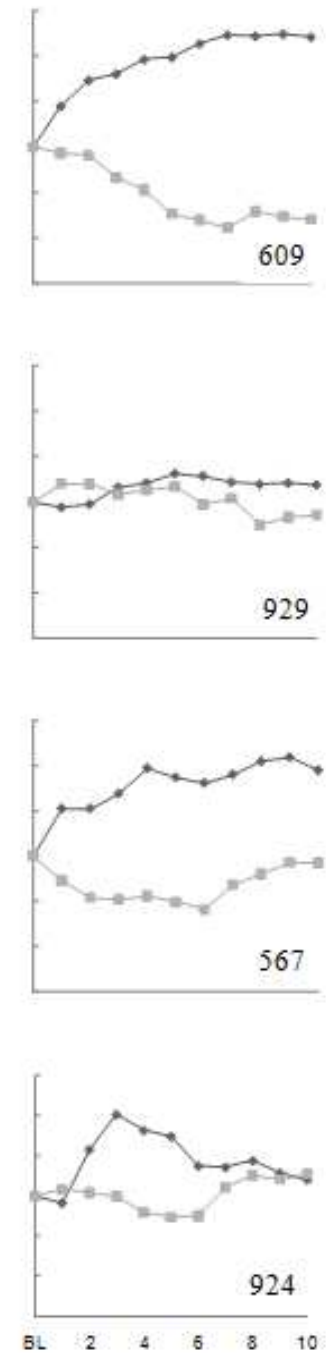

Figure 31. Log ratio of response rate as a proportion of the same-component response rate in the last 6 sessions of history building for the first 10 sessions for each pigeon in each experiment. The darker diamonds show the data for Component $\mathrm{A}$, and the lighter squares show the data for Component B. 


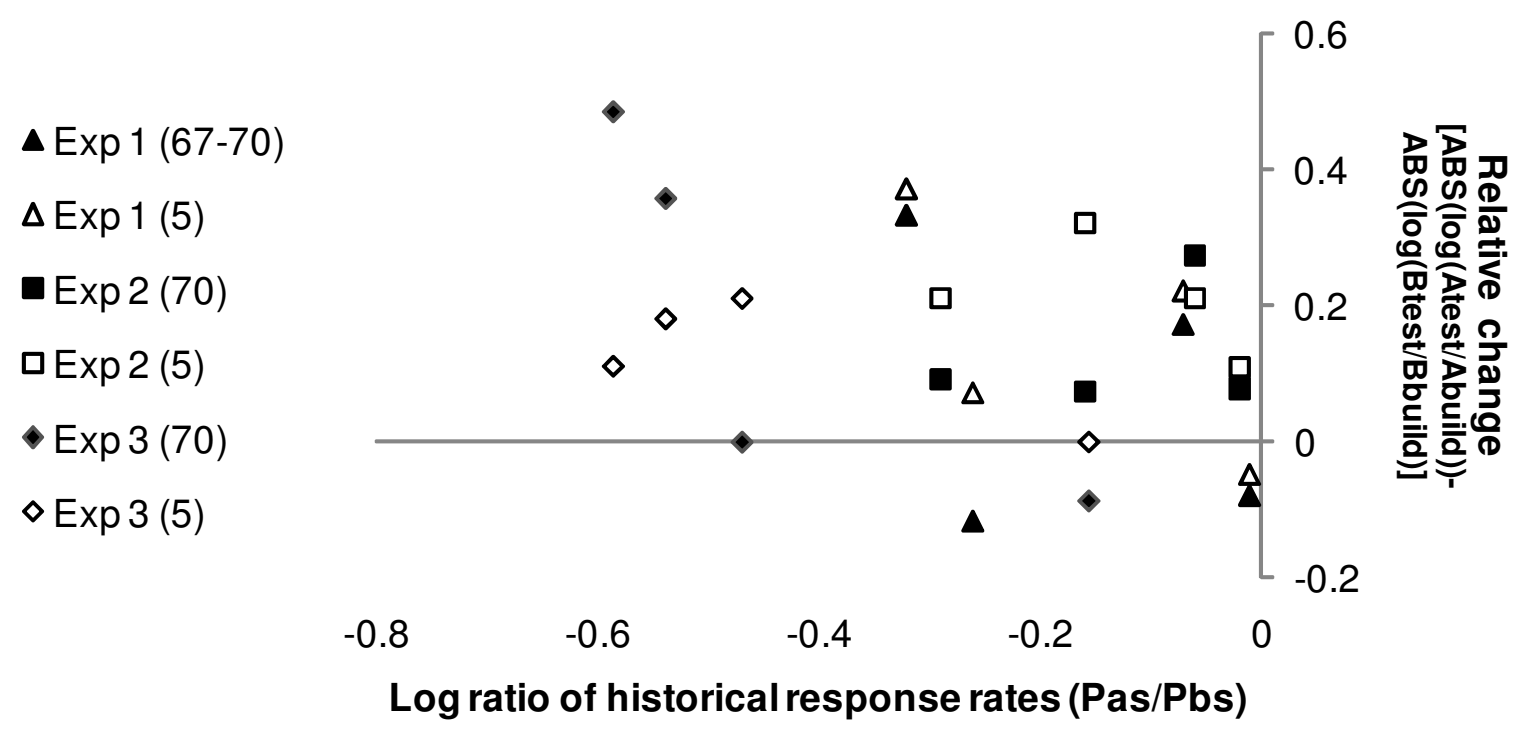

Figure 32. Absolute difference scores for each pigeon as a function of the log ratio of historical response rates $[\log (\mathrm{Pas} / \mathrm{Pbs})]$. Open symbols represent data from the first 5 sessions of history testing, and closed symbols represent data from the first 70 sessions (the first 67 sessions for pigeon 975).

Effects of remote history can be measured in a number of ways, and research that extends the analysis to features of responding that are affected by remote reinforcement or behavioral histories would add to the understanding of adaptation to new environments, response strength, and behavioral persistence. In the current study effects on subsequent responding were more difficult to characterize than they have been in many previous studies of behavioral history. Nonetheless, understanding of how historical context and changes in context affect subsequent behavior are important, as these variables exist and affect behavior both in and out of the laboratory. The current study attempted to address this difficulty in interpreting history effects by using additional measures of persistence, thereby suggesting new potential lenses through which effects of behavioral history may be viewed.

\section{Implications for Theory and Application}

In contrast with several previous studies (e.g., Blackman, 1968; Doughty \& Lattal, 2001; Freeman \& Lattal, 1992; Lattal, 1989; Metzger, 1992; Nevin, 1974, Experiment 5; Nevin et al., 2001; Okouchi \& Lattal, 2006; Reed \& Doughty, 2005; Tatham et al., 1993; Urbain et al., 1978), the current study did not find lower-rate responding to be more persistent than higher-rate responding. Additionally, the study posed challenges to behavioral momentum theory (Nevin \& Grace, 2001). Multiple-schedule components with a higher overall rate of reinforcement were not more resistant to change than components with a lower overall rate of reinforcement. As has been argued by many authors in discussions of previous studies of responding under multiple-concurrent schedules, it seemed that historical patterns of responding across alternatives affected subsequent responding (cf. Gibbon, 1995; Grace \& Savastano, 1997; McDevitt \& Bell, 2008; Nevin \& Grace, 2000; Williams \& Bell, 1996, Experiment 2; Zentall, Weaver, \& Sherburne, 1996). Future research may clarify relations between historical events and subsequent responding by manipulating the context of reinforcement in the historical condition, controlling for differences in the structure of the functional response unit across components and conditions, and by testing under a variety of conditions, including various schedules of reinforcement. In addition to clarifying issues of behavioral persistence and the functional response unit, further research may also contribute to the effective application of behavioral principals in clinically and socially relevant contexts.

The relation between reinforcement rate and resistance to change has been investigated in a variety of settings with individuals with developmental disabilities (e.g., Ahearn, Clark, Gardenier, Chung, \& Dube, 2003; Dube \& McIlvane, 2001; Dube, McIlvane, Mazzitelli, \& McNamara, 2003; Mace et al., 1990). The general finding from the behavioral momentum literature, that behavior with a history of a higher rate of reinforcement is more resistant to change, has been supported in translational and applied studies with people with developmental disabilities. The analysis of effects of reinforcement and behavioral history on subsequent clinically, educationally, and vocationally 
significant behavior is especially relevant in the design of treatment for individuals with developmental disabilities. Two persistent difficulties related to the issue of behavioral persistence in the treatment of persons with developmental disabilities are the (a) adaptation of responding (differently from a previous condition) when the contingencies change, and the (b) generalization of responding (similarly to a previous condition) when the environment changes. A third difficulty in clinical and educational intervention is the (c) persistence of responding (in performing a job-related task, for example), despite a temporary change in the level of supervision or schedule of reinforcement. Problems of suboptimal adaptation, generalization, and persistence not only are relevant to the treatment of individuals with developmental disabilities, but also are relevant to treatment of those with other disorders (e.g., attention-deficit hyperactivity disorder, obsessive-compulsive disorder), and to individuals who require no clinical intervention, but whose performance could be improved by more effective training and management. To this end, an improved understanding of historical factors that increase the likelihood that responding will adapt, generalize, or persist in a changed environment could be useful in designing superior clinical, instructional, and managerial practices. The study of behavioral history is critical to this understanding.

In light of the current study, it appears that response rate in an historical condition is not an important factor in determining the rate of change of response rate in a subsequent condition. The particular schedule that generates historical response rates, however, does appear to be an important factor in determining the rate of change of response rate. Improved understanding of how these schedules affect subsequent behavior is important for clinical, educational, and industrial application of behavior analysis. Although there is much evidence in the literature that reinforcement rate in an historical condition is an important factor in determining the rate of change in response rate in a subsequent condition, the current set of experiments does not replicate the general finding that behavior maintained in a context with a higher overall rate of reinforcement leads to greater resistance to change (e.g., Nevin et al., 1990). Nevin and his colleagues argue that the stimulus-reinforcer relation is the critical determinant of behavioral persistence in a given context. A critical question, in light of the absence of a behavioral-momentum type finding in Experiments 1 and 2, is "what defines context:" in what ways must the historical and test conditions be similar to one another for the general findings from behavioral momentum theory to hold? From the practitioner's standpoint, the specification of conditions under which more or less behavioral persistence will occur is of utmost importance. When should there, and when should there not be a concern that a treatment will lead to increased resistance to change? The identification of these conditions is critical to identifying limits to the generality of findings from studies of behavioral history and resistance to change.

Final Remarks

The present set of experiments has provided a novel approach to investigating effects of historical response rate on behavioral persistence: reinforcement context, rather than contingencies that directly controlled response rate, was used with equal rates of reinforcement to generate high- and low-rate responding. There was no evidence in this study that lower-rate responding was more persistent. To the contrary, responding in the component designed to generate higher-rate responding was generally more resistant to change. These findings support and extend previous literature in which there is evidence that factors other than reinforcement rate and response rate contribute to behavioral persistence (e.g., Doughty \& Lattal, 2001; Grace, et al., 1998; Podlesnik et al., 2006; Podlesnik \& Shahan, 2008). These factors include features of the particular schedule of reinforcement used to build the behavioral history, and the topography (or organization) of the resulting functional response unit. Response organization and behavioral persistence appear to intersect in an important way, and this intersection deserves further exploration (e.g., Podlesnik et al., 2006; Shull et al., 2004). Finally, it seems that attempting to bridge empirically what researchers have learned from the study of behavioral history and behavioral momentum is potentially a fruitful endeavor. 


\section{References}

Ahearn, W. H., Clark, K. M., Gardenier, N. C., Chung, B. I., \& Dube, W. V. (2003). Persistence of stereotypic behavior: Examining the effects of external reinforcers. Journal of Applied Behavior Analysis, 36, 439 448.

Belke, T. W. (1992). Stimulus preference and the transitivity of preference. Animal Learning and Behavior, 20, 401406.

Bell, M. C. (1999). Pavlovian contingencies and resistance to change in a multiple schedule. Journal of the Experimental Analysis of Behavior, 72, 81-96.

Bell, M. C., \& Williams B. A. (2002).Preference and resistance to change in concurrent variable-interval schedules. Animal Learning and Behavior, 30, 34-42.

Blackman, D. (1968). Conditioned suppression or facilitation as a function of the behavioral baseline. Journal of the Experimental Analysis of Behavior, 11, 53-61.

Catania, A. C. (1963). Concurrent performances: Reinforcement interaction and response independence. Journal of the Experimental Analysis of Behavior, 6, 253-263.

Cole, M. R. (2001). The long-term effect of high- and low-rate responding histories on fixed interval responding in rats. Journal of the Experimental Analysis of Behavior, 75, 43-54.

da Silva, S.P., Maxwell, M.E., \& Lattal, K.A. (2008). Concurrent resurgence and behavioral history. Journal of the Experimental Analysis of Behavior, 90, 313-331.

Dickson, C. A., Hayashi, Y., Lightner, A., \& Lattal, K. A. (2009, May). Effects of feedback following a spatially defined response in pigeons. Paper presented at the $35^{\text {th }}$ annual convention of the Association for Behavior Analysis International, Phoenix, AZ.

Doughty, A. H., \& Lattal, K. A. (2001). Resistance to change of operant variation and repetition. Journal of the Experimental Analysis of Behavior, 76, 195-215.

Dube, W. V., \& McIlvane, W. J. (2001). Behavioral momentum in computer-presented discriminations in individuals with severe mental retardation. Journal of the Experimental Analysis of Behavior, 75, 15-23.

Dube, W. V., \& Mcllvane, W. J. (1997). Reinforcer frequency and restricted stimulus control. Journal of the Experimental Analysis of Behavior, 68, 303-316.

Dube, W. V., McIlvane, W. J., Mazzitelli, K., \& McNamara, B. (2003). Reinforcer rate effects and behavioral momentum in individuals with developmental disabilities. American Journal on Mental Retardation, 108, 134-143.

Fath, S. J., Fields, L., Malott, M. K., \& Grossett, D. (1983). Response rate, latency, and resistance to change. Journal of the Experimental Analysis of Behavior, 39, 267-274.

Freeman, T. J. \& Lattal, K. A. (1992). Stimulus control of behavioral history. Journal of the Experimental Analysis of Behavior, 57, 5-15.

Gibbon, J. (1995). Dynamics of time matching: Arousal makes better seem worse. Psychonomic Bulletin \& Review, 2, 208-215.

Grace, R. C., \& Nevin, J. A. (1997). On the relation between preference and resistance to change. Journal of the Experimental Analysis of Behavior, 67, 43-65.

Grace, R. C., \& Savastano, H. I. (1997). Transfer tests of stimulus value in concurrent chains. Journal of the Experimental Analysis of Behavior, 67, 93-115.

Grace, R. C., Schwendiman, J. W., \& Nevin, J. A. (1998). Effects of unsignaled delay of reinforcement on preferences and resistance to change. Journal of the Experimental Analysis of Behavior, 69, 247-261.

Harper, D. (2000). Problems with the concept of force in the momentum metaphor. Behavioral and Brain Sciences, 23,100 . 
Harper, D. N. \& McLean, A. P. (1992). Resistance to change and the law of effect. Journal of the Experimental Analysis of Behavior, 57, 317-337.

Herrnstein, R. J. (1970). On the law of effect. Journal of the Experimental Analysis of Behavior, 13, 243-266.

Hinson, J. M., \& Staddon, J. E. (1978). Behavioral competition: a mechanism for schedule interactions. Science, 202, 432-434.

Holland, J. G. (1958). Counting by humans on a fixed-ratio schedules of reinforcement. Journal of the Experimental Analysis of Behavior, 1, 175-181.

Lattal, K. A. (1974). Combinations of response-reinforcer dependence and independence. Journal of the Experimental Analysis of Behavior, 22, 357-362.

Lattal, K. A. (1989). Contingencies of response rate and resistance to change. Learning and Motivation, 20, 191203.

Lattal, K. A., Reilly, M. P., \& Kohn, J. P. (1998). Response persistence under ratio and interval reinforcement schedules. Journal of the Experimental Analysis of Behavior, 70, 165-183.

LeFrancois J. R., \& Metzger, B. (1993). Low-response conditioning history and fixed-interval responding in rats. Journal of the Experimental Analysis of Behavior, 59, 543-549.

Lopez, F., \& Menez, M. (2005). Effects of reinforcement history on response rate and response pattern in periodic reinforcement. Journal of the Experimental Analysis of Behavior, 83, 221- 241.

Mace, F. C., Lalli, J. S., Shea, M. C., Lalli, E. P., West, B. J., Roberts, M., et al. (1990). The momentum of human behavior in a natural setting. Journal of the Experimental Analysis of Behavior, 54, 163-172.

McDevitt, M. A., \& Williams, B. A. (2003). Arousal, changeover responses, and preference in concurrent schedules. Journal of Experimental Analysis of Behavior, 80, 261-272.

McIlvane W. M., \& Dube, W. V., 2001 Behavioral momentum and multiple stimulus control topographies. Behavioral and Brain Sciences, 23, 109.

McLean, A. P. (1992). Contrast and reallocation of extraneous reinforcers between multiple-schedule components. Journal of the Experimental Analysis of Behavior, 58, 497-511.

McLean, A. P., \& Blampied, N. M. (1995). Resistance to reinforcement change in multiple and concurrent schedules assessed in transition and at steady state. Journal of the Experimental Analysis of Behavior, 63, 1-17.

Metzger, B. A. (1992). Stimulus control of behavioral history and subsequent fixed interval performance. Unpublished doctoral dissertation, West Virginia University, Morgantown.

Nader, M. A., \& Thompson, T. (1987). Interaction of methadone, reinforcement history, and variable-interval performance. Journal of the Experimental Analysis of Behavior, 48, 303-315.

Nevin, J. A. (1974). Response strength in multiple schedules. Journal of the Experimental Analysis of Behavior, 21, 389-408.

Nevin, J. A. (1979). Reinforcement schedules and response strength. In M.D. Zeiler \& P. Harzem (Eds.), Reinforcement and the organization of behaviour (pp. 117-158). Chichester, England: Wiley.

Nevin, J. A. (1992). An integrative model of the study of behavioral momentum. Journal of the Experimental Analysis of Behavior, 57, 301-316.

Nevin, J. A., \& Grace, R. C. (2000). Behavioral momentum and the law of effect. Behavioral and Brain Sciences, $23,73-130$.

Nevin, J. A., Grace, R. C., Holland, S., \& McLean, A. P. (2001). Variable-ratio versus variable- interval schedules: Response rate, resistance to change, and preference. Journal of the Experimental Analysis of Behavior, 76, 43-74.

Nevin, J. A., Mandell, C., \& Yarensky, P. (1981). Response rate and resistance to change in chained schedules. Journal of Experimental Psychology: Animal Behavior Processes, 7, 278-294. 
Nevin, J. A., Tota, M. E., Torquato, R. D., \& Shull, R. D. (1990). Alternative reinforcement increases resistance to change: Pavlovian or operant contingencies. Journal of the Experimental Analysis of Behavior, 53, 359379 .

Okouchi, H. (2003). Stimulus generalization of behavioral history. Journal of the Experimental Analysis of Behavior, 80, 173-186.

Okouchi, H., \& Lattal, K. A. (2006). An analysis of reinforcement history effects. Journal of the Experimental Analysis of Behavior, 86, 31-42

Ono, K., \& Iwabuchi, K. (1997). Effects of histories of differential reinforcement of response rate on variableinterval responding. Journal of the Experimental Analysis of Behavior, 67, 311-322.

Podlesnik, C. A., Jimenez-Gomez, C., Ward, R. D., \& Shahan, T. A. (2006). Resistance to change of responding maintained by unsignaled delays to reinforcement: A response-bout analysis. Journal of the Experimental Analysis of Behavior, 85, 329-347.

Podlesnik, C. A., \& Shahan, T. A. (2008). Response-reinforcer relations and resistance to change. Behavioural Processes, 77, 109-125.

Poling, A. Krafft, K., \& Chapman, L. (1980). d-Amphetamine, operant history, and variable-interval performance. Pharmacology, Biochemistry, and Behavior, 12, 559-562.

Pliskoff, S. S., Shull, R. L., \& Gollub, L. R. (1968). The relation between response rates and reinforcement rates in a multiple schedule. Journal of the Experimental Analysis of Behavior, 11, 271-284.

Reed, P., \& Doughty, A. H. (2005). Within-subject testing of the signaled-reinforcement effect on operant responding as measured by response rate and resistance to change. Journal of the Experimental Analysis of Behavior, 83, 31-45.

Rachlin, H., \& Baum, W. M. (1972). Effects of alternative reinforcement: Does the source matter? Journal of the Experimental Analysis of Behavior, 18, 232-241.

Reynolds, G. S., (1961). Attention in the pigeon. Journal of the Experimental Analysis of Behavior, 4, 203-208.

Roberts, J. E., Tarpy, R. M., \& Lea, S. E. G. (1984). Stimulus-response overshadowing: Effects of signaled reward on instrumental responding as measured by response rate and resistance to change. Journal of Experimental Psychology: Animal Behavior Processes, 10, 244-255.

Schaal, D. W., Shahan, T. A., Kovera, C. A., \& Reilly, M. P. (1998). Mechanisms underlying the effects of unsignaled delayed reinforcement on key pecking of pigeons under variable-interval schedules. Journal of the Experimental Analysis of Behavior, 69, 103-122.

Shahan, T. A., \& Lattal, K. A. (1998). On the functions of the changeover delay. Journal of the Experimental Analysis of Behavior, 69, 141-160.

Shull, R. L., Grimes, J. A., \& Bennett, J. A. (2004). Bouts of responding: The relation between bout rate and the rate of variable-interval reinforcement. Journal of the Experimental Analysis of Behavior, 81, 65-83

Sidman, M. (1960) Tactics of scientific research: Evaluating experimental data in psychology. Basic Books: New York.

Skinner, B. F. (1938). The behavior of organisms. New York: Appleton-Century-Crofts.

Skinner, B. F. (1948). 'Superstition' in the pigeon. Journal of Experimental Psychology, 38, 168-172.

Staddon, J. E. R., \& Simmelhag, V. (1971). The "superstition" experiment: A reexamination of its implications for the principles of adaptive behavior. Psychological Review, 78, 3-43.

Stubbs, D. A., \& Pliskoff, S. S. (1969). Concurrent responding with fixed relative rate of reinforcement. Journal of the Experimental Analysis of Behavior, 6, 887-895.

Tatham, T. A., Wanchisen, B. A., \& Yasenchack, M. P. (1993). Effects of differential-reinforcement-of-low rate schedule history on fixed-interval responding. The Psychological Record, 43, 289-297.

Urbain, C., Poling, A., Millam, J., \& Thompson, T. (1978). $d$-amphetamine and fixed-interval performance: Effects of operant history. Journal of the Experimental Analysis of Behavior, 29, 385-392. 
Wanchisen, B. A., Tatham, T. A., \& Mooney, S. E. (1989). Variable-ratio conditioning history produces high- and low-rate fixed-interval performance in rats. Journal of the Experimental Analysis of Behavior, 52,167-179.

Weiner, H. (1964). Conditioning history and human fixed-interval performance. Journal of the Experimental Analysis of Behavior, 7, 383-385.

Weiner, H. (1969). Conditioning history and the control of human avoidance and escape responding. Journal of the Experimental Analysis of Behavior, 12, 1039-1043.

Williams, B. A., \& Bell M. C. (1996). Changeover behavior and preference in concurrent schedules. Journal of the Experimental Analysis of Behavior, 65, 513-526.

Williams, B. A., Ploog, B. O., \& Bell, M. C. (1995). Stimulus devaluation and extinction of chain schedule performance. Animal Learning \& Behavior, 23, 104-114.

Zentall, T. R., Weaver, J. E., \& Sherburne L. M. (1996). Value transfer in concurrent-schedule discriminations by pigeons. Animal Learning and Behavior, 24, 401-409. 
Appendices

Appendix A: Component Sequences for History Building and History Testing

Component Sequence

32 components

Sequence 1

Sequence 2

Sequence 3

Sequence 4

Sequence 5

Sequence 6

28 components

Sequence 1

Sequence 2

Sequence 3

Sequence 4

Sequence 5

Sequence 6

24 components

Sequence 1

Sequence 2

Sequence 3

Sequence 4

Sequence 5

Sequence 6
01101110010010011101010110001100

00110001110110001110011000110110

01011100110010100011100101101001

11001001100111000110010010101110

11001011000101100101001110001101

10010011011001011000101011100011

0110111001001001110101011000

0011000111011000111001100011

0101110011001010001110010110

1100100110011100011001001011

1100101000110110010100111001

1001001101100101100010101110

011011100100100111000101

001100011101100011100110

010111001100101000111001

110010011001110001101100

110010110001011001010110

100100110110010110001011

Note. The characters " 0 " and " 1 " denote the Left and Right components as described in the procedure sections for the individual experiments. 


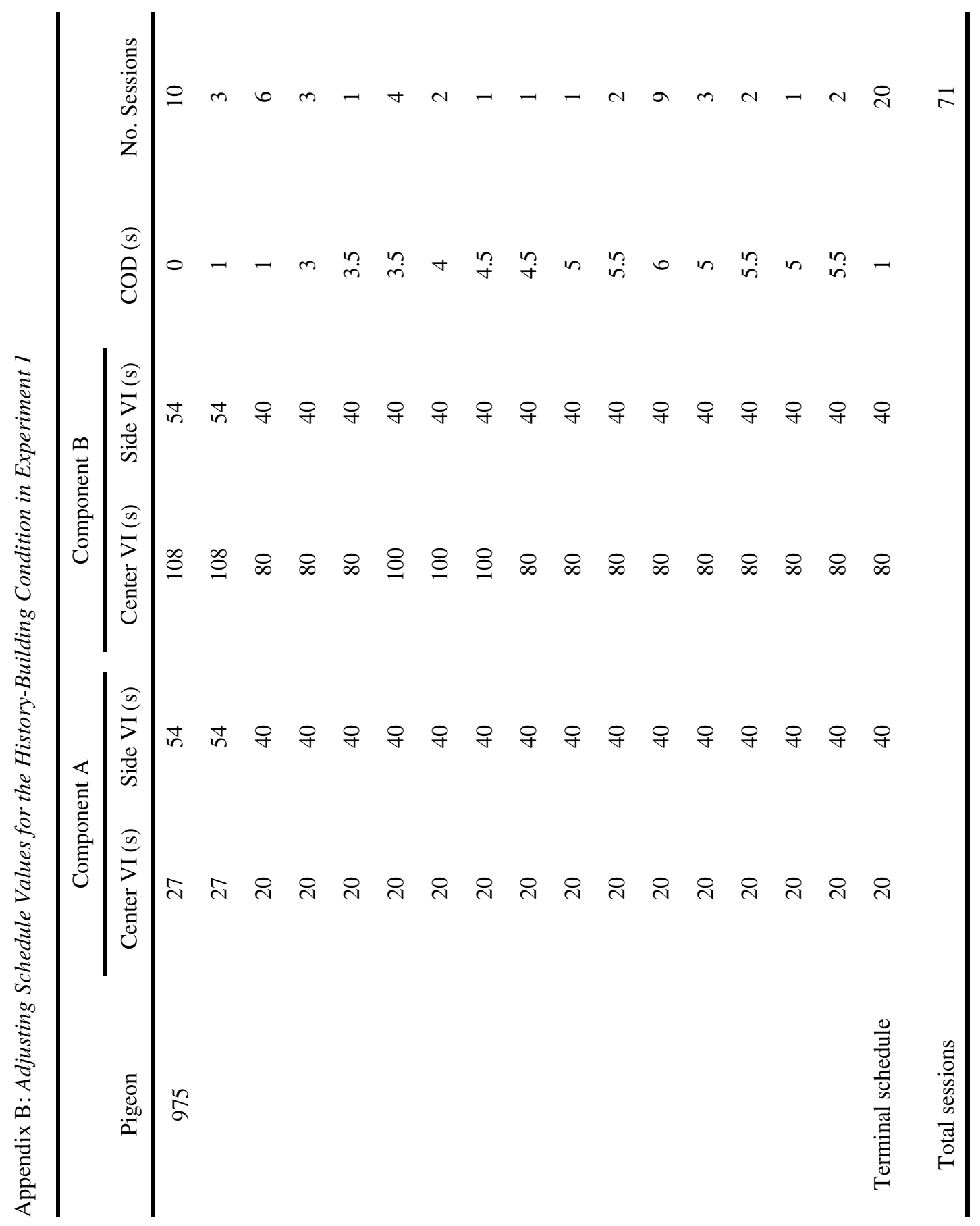




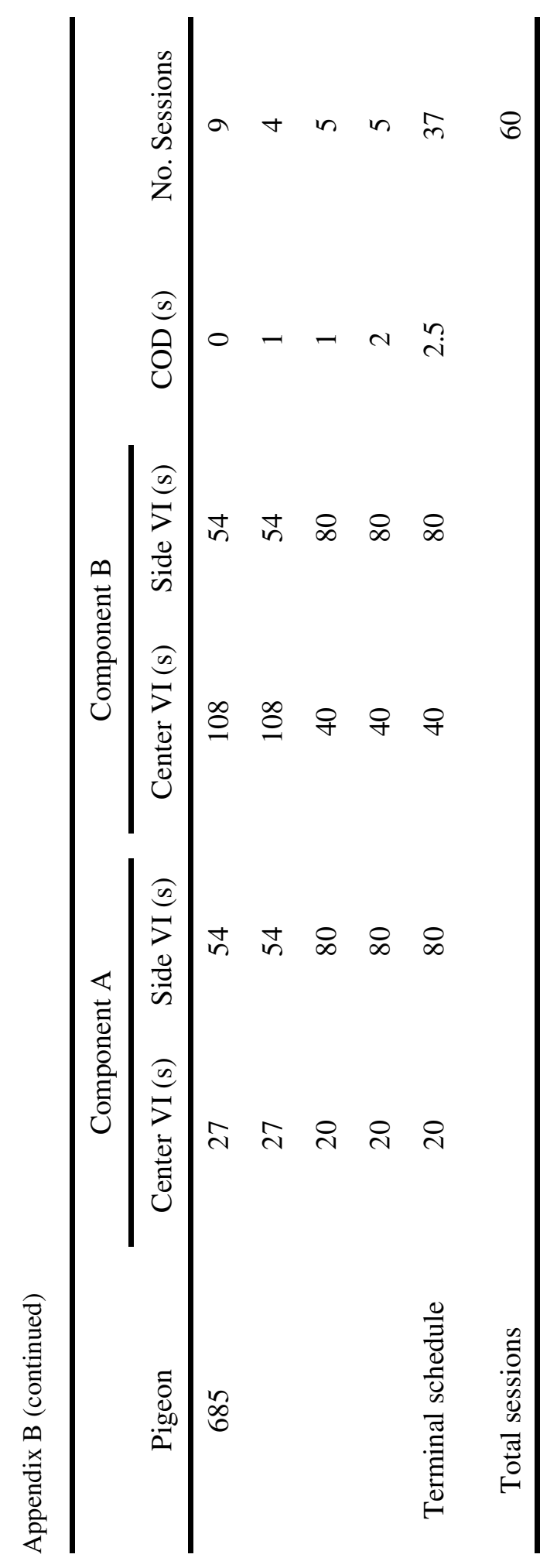




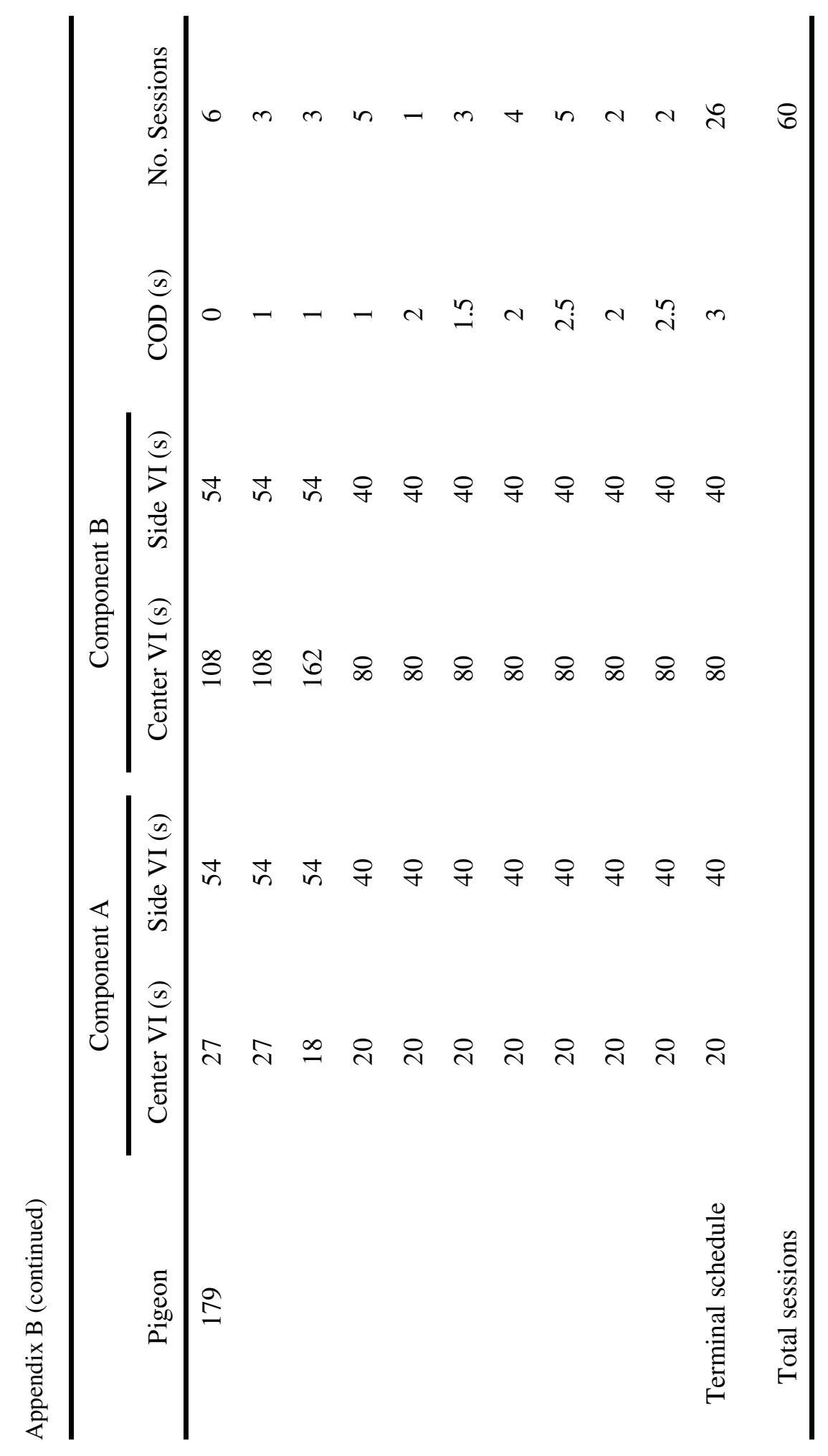




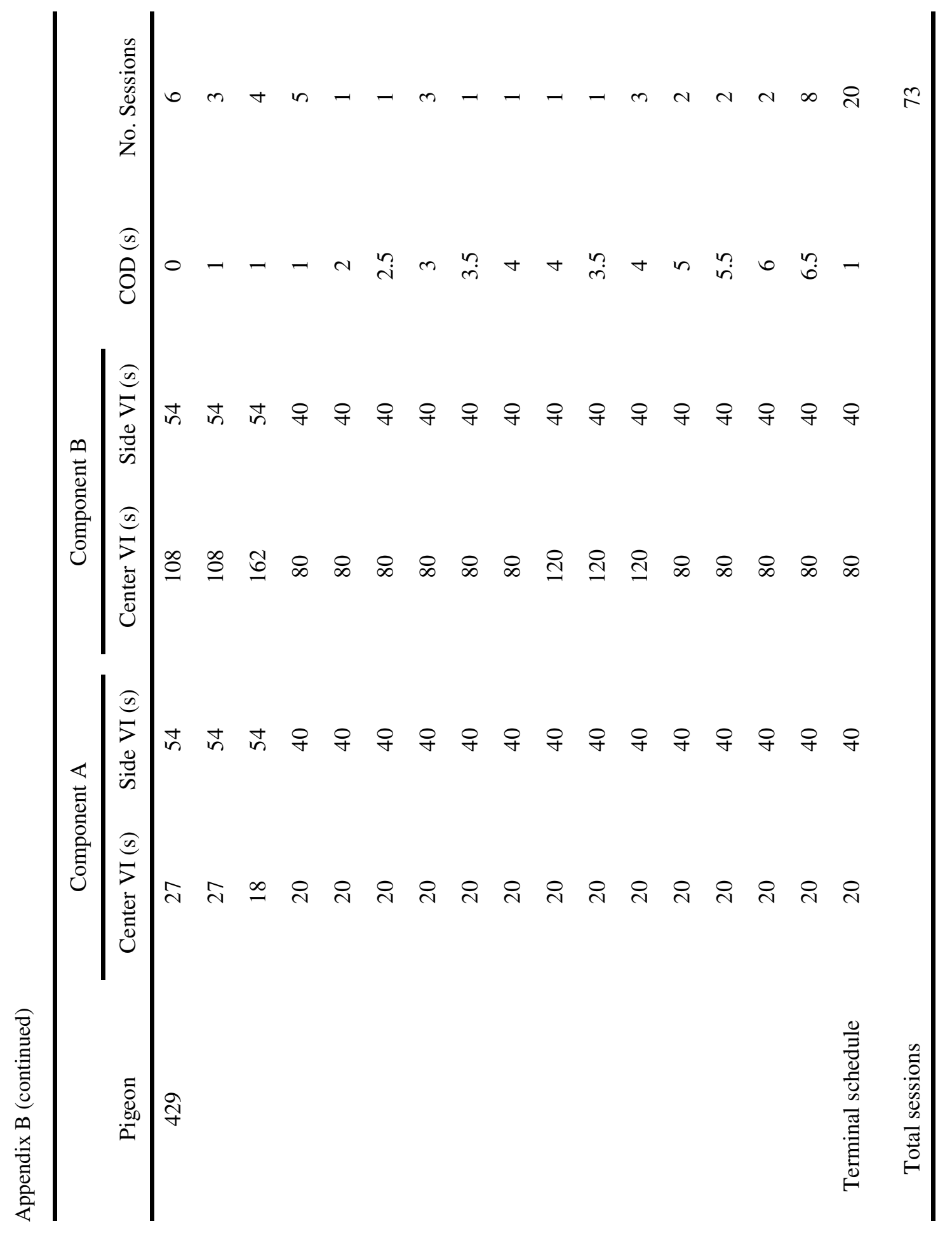


Ð

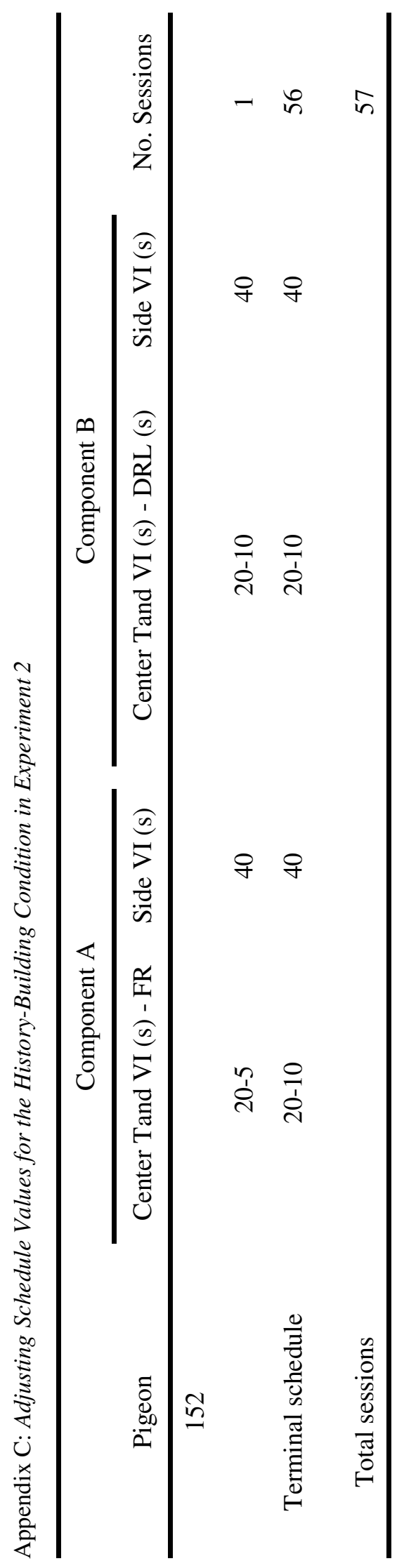


$\infty$

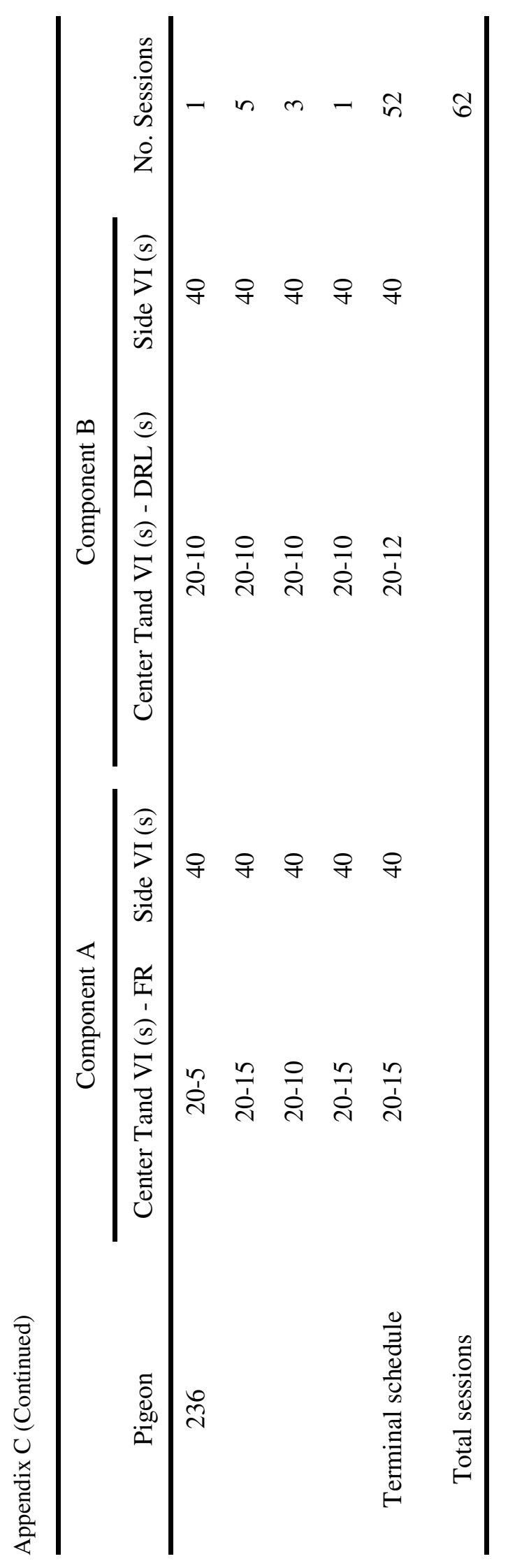


$\infty$

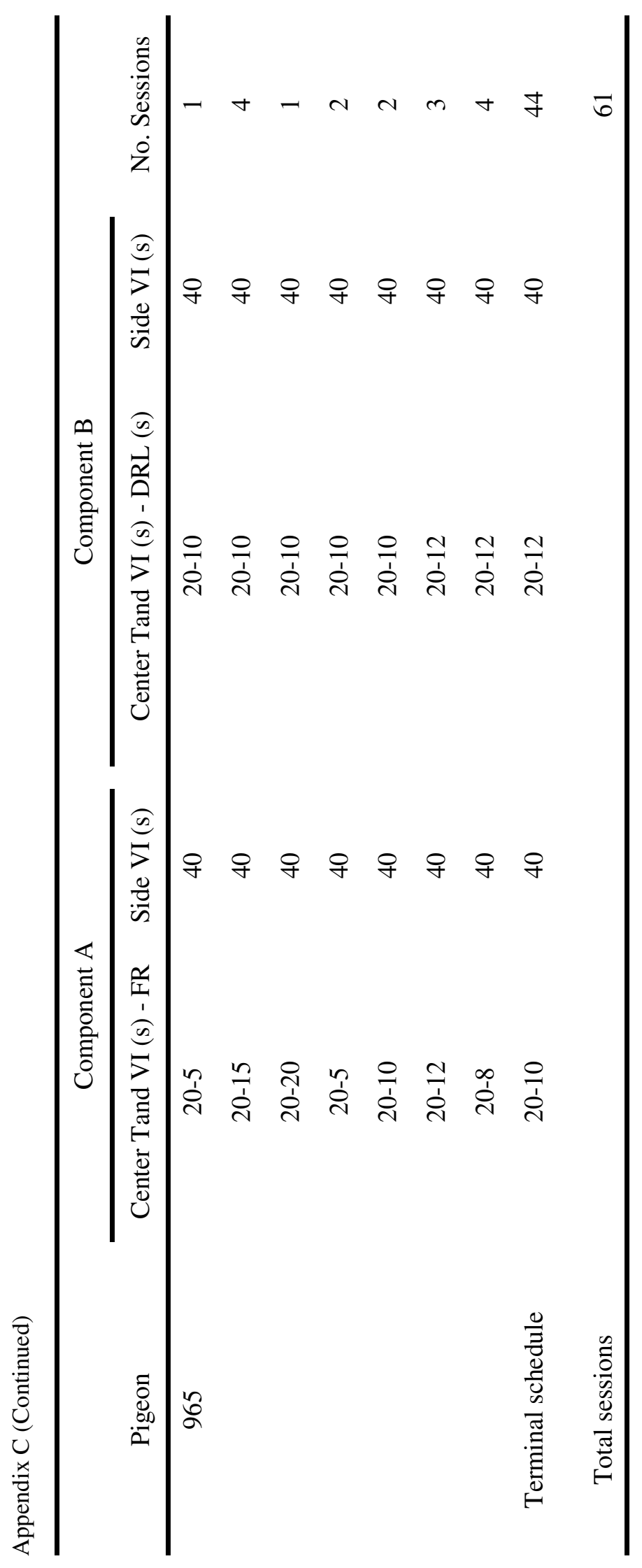




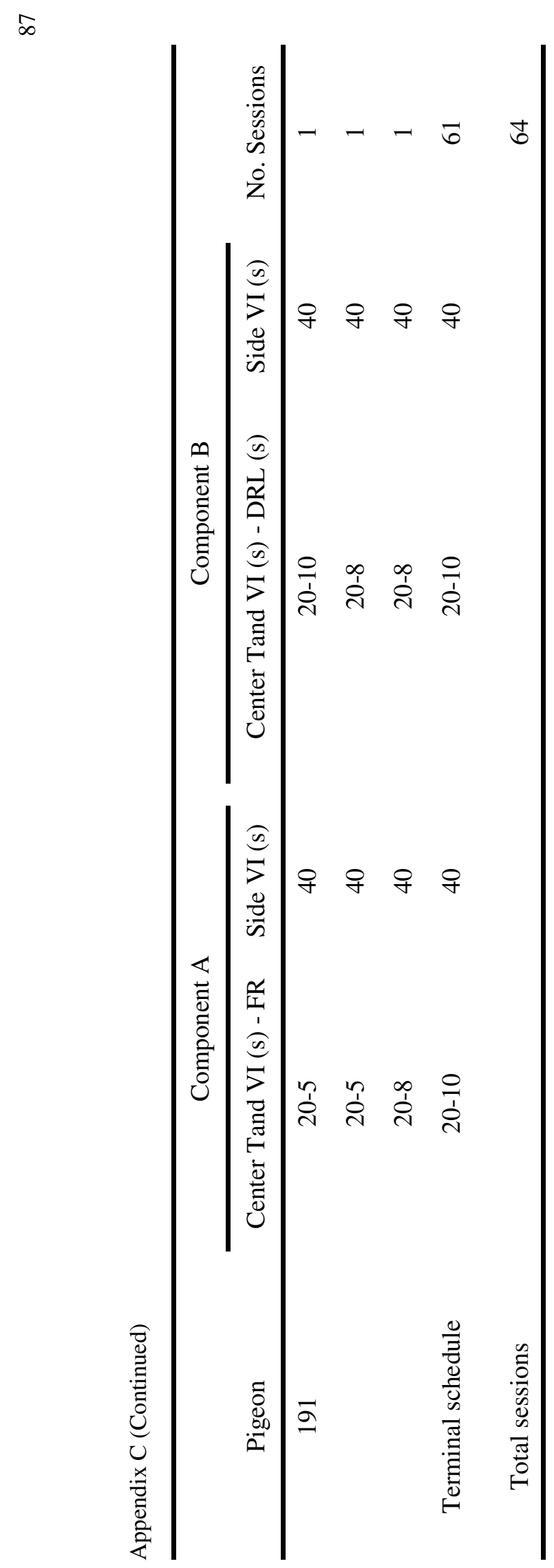


Appendix D: Adjusting Schedule Values for the History-Building Condition in Experiment 3

\begin{tabular}{|c|c|c|c|c|c|}
\hline \multirow[b]{2}{*}{ Pigeon } & & \multicolumn{2}{|c|}{ Component A } & \multirow{2}{*}{$\frac{\text { Component B }}{\text { VI (s) }}$} & \multirow[b]{2}{*}{ No. Sessions } \\
\hline & & VI or VT (s) & $\% \mathrm{VT}$ & & \\
\hline \multirow[t]{4}{*}{609} & & 30 & 75 & 30 & 11 \\
\hline & & 30 & 90 & 30 & 10 \\
\hline & Terminal schedule & 30 & 100 & 30 & 41 \\
\hline & Total sessions & & & & 62 \\
\hline \multirow[t]{4}{*}{929} & & 30 & 75 & 30 & 10 \\
\hline & & 30 & 90 & 30 & 5 \\
\hline & Terminal schedule & 30 & 100 & 30 & 45 \\
\hline & Total sessions & & & & 60 \\
\hline \multirow[t]{4}{*}{567} & & 30 & 75 & 30 & 11 \\
\hline & & 30 & 90 & 30 & 5 \\
\hline & & 30 & 100 & 30 & 35 \\
\hline & Terminal schedule & 30 & 95 & 30 & 20 \\
\hline & Total sessions & & & & 70 \\
\hline \multicolumn{6}{|l|}{924} \\
\hline & Terminal schedule & 30 & 75 & 30 & 60 \\
\hline & Total sessions & & & & 60 \\
\hline
\end{tabular}

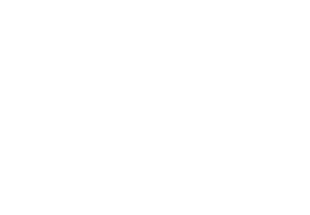

